\title{
Nonlinear stochastic differential games involving a major player and a large number of collectively acting minor agents*
}

\author{
Rainer Buckdahn"1,3, Juan $\mathrm{Li}^{2}$, Shige Peng ${ }^{3}$ \\ ${ }^{1}$ Laboratoire de Mathématiques LMBA, CNRS-UMR 6205, Université de Bretagne Occidentale, \\ 6, avenue Victor-le-Gorgeu, CS 93837, 29238 Brest cedex 3, France. \\ ${ }^{2}$ School of Mathematics and Statistics, Shandong University (Weihai), Weihai 264209, P. R. China.; \\ ${ }^{3}$ School of Mathematics, Shandong University, Jinan 250100, P. R. China. \\ E-mails: rainer.buckdahn@univ-brest.fr, juanli@sdu.edu.cn,peng@sdu.edu.cn.
}

August 15, 2013

\begin{abstract}
The purpose of this paper is to study 2-person zero-sum stochastic differential games, in which one player is a major one and the other player is a group of $N$ minor agents which are collectively playing, statistically identical and have the same cost-functional. The game is studied in a weak formulation; this means in particular, we can study it as a game of the type "feedback control against feedback control". The payoff/cost functional is defined through a controlled backward stochastic differential equation, for which driving coefficient is assumed to satisfy strict concavity-convexity with respect to the control parameters. This ensures the existence of saddle point feedback controls for the game with $N$ minor agents. We study the limit behavior of these saddle point controls and of the associated Hamiltonian, and we characterize the limit of the saddle point controls as the unique saddle point control of the limit mean-field stochastic differential game.
\end{abstract}

AMS Subject classification: 93E05, 90C39, 60H10, 60H30.

Keywords: Stochastic differential game; 2-person zero-sum stochastic differential game; backward stochastic differential equation; saddle point control.

\section{Introduction}

In this paper we study a particular type of 2-person zero-sum stochastic differential games, where one player is a major one, who plays against a group of $N$ collectively acting minor agents which each of them participate with the same percentage and are statistically identical. We study the stochastic differential game in its weak formulation and with a pay-off/cost-functional given through a backward stochastic differential equation (BSDE), which allows to consider the game of the type "feedback control against feedback control". Under suitable assumptions on the driving coefficient of the BSDE we show for the game with $N$ minor agents the existence of saddle point feedback controls, which can be characterized as Stackelberg feedback strategy, where the major player is the leader and the collectively acting minor agents are the follower. We investigate the limit of these saddle point feedback controls and of the associated Hamiltonian of stochastic differential game and characterize the limit saddle point controls as unique saddle point controls of the limit stochastic differential game which turns out to be of mean-field type.

${ }^{*}$ The work has been supported by the NSF of P.R.China (Nos. 11071144, 11171187, 11222110), Shandong Province (Nos. BS2011SF010, JQ201202), Program for New Century Excellent Talents in University (NCET, 2012), 111 Project (No. B12023). Juan $\mathrm{Li}$ is the corresponding author. 
Mean-field stochastic differential games obtained as limit of stochastic differential games between $N$ statistically identical players were studied by Lasry and Lions in a series of pioneering papers ([12, [13, [14, 15]). In their papers they investigated so-called approximate Nash equilibria, obtained as distributed closed-loop strategies given by solving the limit problem. This limit problem consists of a coupled system, formed by a Hamilton-Jacobi-Bellman equation and an equation of Kolmogorov type, the first one with a terminal and the second one with an initial condition. In subsequent works several authors studied different applications coming from such different domains as, for instance, Statistical Mechanics and Physics, finance and management of exhaustible resources. On the other hand, motivated by problems occurring in large communication networks but also in stochastic differential games involving a large number of players, Huang, Malhamé and Caines [11] introduced a similar concept, that of Nash Certainty Equivalence.

The problem of investigating stochastic differential games with a large number of players or with one major player at one side and $N$ minor players on the other side, which participate each of them in the same proportion and in a symmetric way at the game, leads in the limit, as $N$ tends to infinity, to an averaging over the minor players and, thus, to a mean-field limit game. Such a problem, without the control part, relates with the McKean-Vlasov theory of chaos propagation (we refer the reader to the scholarly paper by Sznitman [18]). In [5] and 6] Carmona and Delarue, and Carmona, Delarue and Lachapelle, respectively, study approximate Nash-equilibria for $N$-person non-zero stochastic games with mean-field interaction between the players and they discuss the limit behavior as the number of players converges to infinity. In [5] the authors embed the Mean-Field Game strategy developed by Lasry and Lions, in an analytical approach into a purely probabilistic framework, which transforms the strongly coupled system consisting of a HamiltonJacobi-Bellman equation with terminal condition and a Kolmogorov-type equation with initial condition, considered by Lasry and Lions, into a strongly coupled forward-backward stochastic differential equation (FBSDE) of McKean-Vlasov type. The authors of [5] show that the solutions of this FBSDE together with the associated FBSDE value function allow to obtain a set of distributed strategies which turn out to be an $\varepsilon_{N}$-approximate Nash equilibrium for the $N$-person non-zero sum stochastic differential game, where $\varepsilon$ converges to zero as $N$ tends to infinity. Such a kind of argument, but for simpler models can be also found in [1] an in [4. In [6] Carmona, Delarue and Lachapelle make comparing studies between stochastic differential games with mean-field interactions on one side and the characterization of optimal strategies for the associated mean-field linear-quadratic McKean-Vlasov stochastic control problem on the other side.

In their recent work [16] Nourian and Caines study with a different approach that those chosen by Lasry and Lions and Carmona and Delarue $\varepsilon$-Mean-Field games. They consider $N+1$-person non-zero sum stochastic differential games with one major player and $N$ symmetric minor players. The stochastic dynamics are non-linear and with mean-field interaction, the forward equation for each player is driven by its own Brownian motion and each player control only his own dynamics and his own running cost; the running cost are non-linear and with mean-field interaction. The study of $\varepsilon_{N}$-Nash equilibriums for the $N+1$ person game for large $N$ leads the authors to a strongly coupled stochastic mean-field system composed of a stochastic Hamilton-Jacobi-Bellman equation with terminal condition and two McKean-Vlasov equations with stochastic coefficients, describing the state of the major player as well as the measure determining the mean-field behavior of the minor agents. Let us emphasize that the paper [16] represent an extension to the framework of non-linear mean-field stochastic differential equations, which was preceded by papers by Huang [10] but also by Huang together with Caines and Malhamé [1] and with Nourian [17, in order to mention only these important works of a longer list of papers.

In the present work we study a somehow different framework which, although is related with the works discussed above, in particular with [16]. But unlike [16] we consider the $N$ minor agents as collectively acting, with a common cost functional. This allows to consider the game as 2-person zero-sum stochastic differential game. Moreover, it will be studied in a weak form. Stochastic differential games in the weak form have been studied by Hamadène and Lepeltier $[9$ but also by Hamadène in different works, see, e.g., [7] and [8].

In order to be more precise, for independent Brownian motions $\tilde{W}^{0}, \tilde{W}^{1}, \ldots, \tilde{W}^{N}$ and given initial 
positions $x^{(N)}=\left(x_{0}, x_{1}, \ldots, x_{N}\right)$ we consider the weak solution $X^{(N)}=\left(X^{0, N}, X^{1, N}, \ldots, X^{N, N}\right)$ of the following system of dynamics with given feedback controls $u=u\left(X^{(N)}\right)$ - for the major player and $v^{(N)}(=$ $\left.\left(v^{1}, \ldots, v^{N}\right)\right)=v\left(X^{(N)}\right)$ for the $N$ collectively acting minor agents:

$$
\begin{aligned}
& d X_{s}^{0, N}=\sigma_{0}\left(X^{0, N}\right) d \tilde{W}_{s}^{0}+\frac{1}{N} \sum_{\ell=1}^{N} b_{0}\left(X_{s}^{0, N}, X_{s}^{\ell, N}, Z_{s}^{0, N}\right) u_{s} d s, \quad X_{t}^{0, N}=x_{0}, \\
& d X_{s}^{j, N}=\sigma_{1}\left(X^{j, N}\right) d \tilde{W}_{s}^{j}+\frac{\varepsilon_{N}}{N} \sum_{\ell=1}^{N} b_{1}\left(X_{s}^{0, N}, X_{s}^{\ell, N}, Z_{s}^{j, N}\right) v_{s}^{\ell} d s, \quad X_{t}^{j, N}=x_{j},
\end{aligned}
$$

$1 \leq j \leq N$, and we associate the nonlinear payoff/cost functional $J(t, x ; u, v):=Y_{t}^{N}$ defined through the following BSDE:

$$
\begin{aligned}
d Y_{s}^{N} & =-\frac{1}{N} \sum_{\ell=1}^{N} f\left(X_{s}^{0, N}, X_{s}^{\ell, N}, Y_{s}^{N}, Z_{s}^{0, N}, Z_{s}^{\ell, N}, u_{s}, v_{s}^{\ell}\right) d s+\sum_{\ell=0}^{N} Z_{s}^{\ell, N} d \tilde{W}_{s}^{\ell}, s \in[t, T], \\
Y_{T}^{N} & =\frac{1}{N} \sum_{\ell=1}^{N} \Phi\left(X_{T}^{0, N}, X_{T}^{\ell, N}\right) .
\end{aligned}
$$

We see that here the major player can control his own dynamics $X^{0, N}$, and each of the minor agents controls his dynamics but also those of all the other minor agents and, together with the major player; moreover, all players can control the pay-off/cost-functional $J(t, x ; u, v)$. While the objective of the major player is to maximize $J(t, x ; u, v)$, the collectively acting minor agents want to minimize their common cost functional $J(t, x ; u, v)$.

The choice of the weak formulation of the problem of stochastic differential games allows to shift with the help of a Girsanov transformation the doubly controlled drift terms of the dynamics of the game with $N$ collectively acting minor players into the BSDE defining the pay-off/cost functional. This reduces the study of the limit behavior of the game and of the saddle point feedback controls for this game to the investigation of the limit behavior of the corresponding BSDEs with non-feedback controls and the limiting Mean-Field BSDE. In order to guarantee the existence of saddle point controls we impose on the driving coefficient $f$ of the BSDE a strict concavity-convexity assumption with respect to the control parameters $(u, v)$. The specificity of our approach using the Girsanov transformation necessitates the factor $\varepsilon_{N}$ which is supposed to be of order $O\left(N^{-3 / 4}\right)$, as $N \rightarrow+\infty$.

The problem of a limit approach for mean-field BSDEs as well as mean-field BSDEs themselves were studied by the authors in [3] (together with Djehiche) and in [2], but unlike here without controls.

The paper is organized as follows: In Section 2 a short recall for the convergence of the above system (1.1) and (1.2) in the case without control will be given. In Section 3 the stochastic differential game with one major player and $N$ collectively acting minor agents is introduced, the assumptions on the coefficients are given and the existence of saddle points controls which are of feedback form is discussed. They are characterized as a Stackelberg feedback strategy. This characterization admits estimates for the saddle point feedback controls which will be used in what follows. Section 5 is devoted to the study of the limit mean-field game. For this the convergence of the saddle point controls for the game with $N$ minor agents is proved and the limit controls are shown to be the unique saddle point controls of the limit game. In order to improve the readability of the work, the proofs of several lemmas have been postponed to the Appendix.

\section{Preliminaries. The $\mathrm{N}+1$ players system without control}

In this short section we consider first briefly the case of a "stochastic differential game with $N$ minor agents" without control and recall its limit behavior. We restrict for this to the (particular) case we will need for our discussion in the Sections 3 and 4. The more interested reader is referred to [18], and for the BSDE part, for instance, to [3]. 
Let $(\Omega, \mathcal{F}, P)$ be a complete probability space, endowed with a sequence of independent $d$-dimensional Brownian motions $W^{j}=\left(W_{s}^{j}\right)_{s \in[0, T]}, j \geq 0$, where $T>0$ is an arbitrarily fixed time horizon. We denote by $\mathbb{F}=\left(\mathcal{F}_{t}\right)_{t \in[0, T]}$ the filtration generated by $W^{j}, j \geq 0$, and augmented by the $P$-null sets. Given bounded Lipschitz coefficients $b_{0}: \mathbb{R}^{d} \times \mathbb{R}^{d} \rightarrow \mathbb{R}^{d}, \sigma_{0}: \mathbb{R}^{d} \times \mathbb{R}^{d} \rightarrow \mathbb{R}^{d \times d}, b_{1}: \mathbb{R}^{d} \times \mathbb{R}^{d} \times \mathbb{R}^{d} \rightarrow \mathbb{R}^{d \times d}$ and $\sigma_{1}: \mathbb{R}^{d} \times \mathbb{R}^{d} \times \mathbb{R}^{d} \rightarrow \mathbb{R}^{d \times d}$, we consider for an arbitrarily chosen initial time $t \in[0, T]$ and initial positions $x_{0}, \ldots, x_{N} \in \mathbb{R}^{d}$ the system of $N \geq 2 d$-dimensional coupled stochastic differential equations (SDEs):

$$
\begin{gathered}
d X_{s}^{0, N}=\frac{1}{N} \sum_{\ell=1}^{N} b_{0}\left(X_{s}^{0, N}, X_{s}^{\ell, N}\right) d s+\frac{1}{N} \sum_{\ell=1}^{N} \sigma_{0}\left(X_{s}^{0, N}, X_{s}^{\ell, N}\right) d W_{s}^{0}, s \in[t, T], \\
X_{t}^{0, N}=x_{0}, \\
d X_{s}^{j, N}=\frac{1}{N} \sum_{\ell=1}^{N} b_{1}\left(X_{s}^{0, N}, X_{s}^{j, N}, X_{s}^{\ell, N}\right) d s+\frac{1}{N} \sum_{\ell=1}^{N} \sigma_{1}\left(X_{s}^{0, N}, X_{s}^{j, N}, X_{s}^{\ell, N}\right) d W_{s}^{j}, s \in[t, T], \\
X_{t}^{j, N}=x_{j}, 1 \leq j \leq N,
\end{gathered}
$$

associated with the backward stochastic differential equation (BSDE):

$$
\begin{aligned}
& d Y_{s}^{N}=-\frac{1}{N} \sum_{\ell=1}^{N} f\left(X_{s}^{0, N}, X_{s}^{\ell, N}, Y_{s}^{N}, Z_{s}^{0, N}, Z_{s}^{\ell, N}\right) d s+Z_{s}^{0, N} d W_{s}^{0}+\sum_{j=1}^{N} Z_{s}^{j, N} d W_{s}^{j}, s \in[t, T], \\
& Y_{T}^{N}=\frac{1}{N} \sum_{\ell=1}^{N} \Phi\left(X_{T}^{0, N}, X_{T}^{\ell, N}\right),
\end{aligned}
$$

where the functions $f: \mathbb{R}^{d} \times \mathbb{R}^{d} \times \mathbb{R} \times \mathbb{R}^{d} \times \mathbb{R}^{d} \rightarrow \mathbb{R}$ and $\Phi: \mathbb{R}^{d} \times \mathbb{R}^{d} \rightarrow \mathbb{R}$ are assumed to be bounded and Lipschitz in all its variables.

Let us now discuss the forward equation (2.1)-(2.2) and the backward equation (2.3) separately.

\subsection{Limit behavior of the forward stochastic system}

The objective of this section is to discuss briefly the limit behavior of the system (2.1) and (2.2):

$$
\begin{aligned}
& \quad d X_{s}^{0, N}=\frac{1}{N} \sum_{\ell=1}^{N} b_{0}\left(X_{s}^{0, N}, X_{s}^{\ell, N}\right) d s+\frac{1}{N} \sum_{\ell=1}^{N} \sigma_{0}\left(X_{s}^{0, N}, X_{s}^{\ell, N}\right) d W_{s}^{0}, s \in[t, T], \\
& \quad X_{t}^{0, N}=x_{0} ; \\
& d X_{s}^{j, N}=\frac{1}{N} \sum_{\ell=1}^{N} b_{1}\left(X_{s}^{0, N}, X_{s}^{j, N}, X_{s}^{\ell, N}\right) d s+\frac{1}{N} \sum_{\ell=1}^{N} \sigma_{1}\left(X_{s}^{0, N}, X_{s}^{j, N}, X_{s}^{\ell, N}\right) d W_{s}^{j}, s \in[t, T], \\
& X_{t}^{j, N}=x_{j}, 1 \leq j \leq N,
\end{aligned}
$$

as $N$ tends to $+\infty$. The limit behavior of such systems as well as the associated limit McKean-Vlasov SDEs have been already largely discussed in the literature. For completeness we first state the following classical existence and uniqueness result.

Proposition 2.1. Under our standard assumptions, that is, the coefficients are bounded and Lipschitz in their variables, we have for any initial datum $\left(t, x^{(N)}\right) \in[0, T] \times\left(\mathbb{R}^{d}\right)^{N+1}, x^{(N)}=\left(x_{0}, x_{1}, \ldots, x_{N}\right)$, the existence and the uniqueness of the solution $X^{(N)}=\left(X^{0, N}, X^{1, N}, \ldots, X^{N, N}\right)$ in the space $\mathcal{S}_{\mathbb{F}}^{2}\left(t, T ; \mathbb{R}^{d}\right)^{N+1}$, where $\mathcal{S}_{\mathbb{F}}^{2}\left(t, T ; \mathbb{R}^{d}\right)$ denotes the space of all continuous $\mathbb{F}$-adapted, $\mathbb{R}^{d}$-valued processes which supremum of the Euclidean norm over the interval $[t, T]$ is square integrable.

Let us now suppose that $\left(x_{j}\right)_{j \geq 0} \subset \mathbb{R}^{d}$ is such that, for some $\bar{x} \in \mathbb{R}^{d}$,

$$
\frac{1}{N} \sum_{j=1}^{N}\left|x_{j}-\bar{x}\right|^{2} \rightarrow 0, \quad \text { as } N \rightarrow+\infty .
$$

In order to justify the choice of this condition, we let $\mathcal{P}_{2}\left(\mathbb{R}^{d}\right)$ be the space of the Borel probability measures on $\mathbb{R}^{d}$ with finite second moments. Wishing that the convergence of the above system (2.1)-(2.2) can be measured in terms of the (Monge-Kantorovich-)Wasserstein distance $d_{2}$ of second order,

$$
d_{2}(\mu, \nu):=\inf \left\{E\left[|\xi-\eta|^{2}\right]: \xi, \eta \in L^{0}\left(\mathcal{F} ; \mathbb{R}^{d}\right) \text { with } P_{\xi}=\mu, P_{\eta}=\nu\right\}, \quad \mu, \nu \in \mathcal{P}_{2}\left(\mathbb{R}^{d}\right) .
$$

We define $\nu^{N}=\frac{1}{N} \sum_{j=1}^{N} \delta_{x_{j}}$, with $\delta_{x_{j}}$ denoting the Dirac measure with mass in $x_{j}$. Given another probability $\nu \in \mathcal{P}_{2}\left(\mathbb{R}^{d}\right)$, the convergence $d_{2}\left(\nu^{N}, \nu\right) \rightarrow 0$, as $N \rightarrow+\infty$, is equivalent to the weak convergence of $\nu^{N}$ to $\nu$ 
as well as that of their second moments. Preferring, for simplicity, the choice $\nu=\delta_{\bar{x}}$, for some $\bar{x} \in \mathbb{R}^{d}$, the convergence $d_{2}\left(\nu^{N}, \nu\right) \rightarrow 0$ is equivalent to (2.4).

The $L^{2}$-limit system of the above systems of SDEs (2.1)-(2.2) (see Proposition 2.2) is given by

$$
\begin{array}{lll}
d \bar{X}_{s}^{0}=b_{0}\left(\bar{X}_{s}^{0}, \mu_{s}\right) d s+\sigma_{0}\left(\bar{X}_{s}^{0}, \mu_{s}\right) d W_{s}^{0}, & s \in[t, T], \bar{X}_{t}^{0}=x_{0}, \\
d \bar{X}_{s}^{j}=b_{1}\left(\bar{X}_{s}^{0}, \bar{X}_{s}^{j}, \mu_{s}\right) d s+\sigma_{1}\left(\bar{X}_{s}^{0}, \bar{X}_{s}^{j}, \mu_{s}\right) d W_{s}^{j}, & s \in[t, T], \bar{X}_{t}^{j}=\bar{x}, j \geq 1,
\end{array}
$$

where

$$
\mu_{s}(d y)=P\left\{\bar{X}_{s}^{1} \in d y \mid \mathcal{F}_{T}^{W^{0}}\right\}
$$

is the conditional distribution law of $\bar{X}_{s}^{1}$ knowing the $\sigma$-field $\mathcal{F}_{T}^{W^{0}}$ which is generated by $W^{0}$ over the time interval $[0, T]$ and augmented by all $P$-null sets.

Given a bounded measurable function $h$ over $\mathbb{R}^{d}$ we use the notation $h\left(\mu_{s}\right)=\int_{\mathbb{R}^{d}} h(x) \mu_{s}(d x)$. By $L$ we denote the second order operator

$$
L[\mu, x] \varphi\left(x^{\prime}\right):=\frac{1}{2} \operatorname{tr}\left(\sigma_{1} \sigma_{1}^{*}\left(x, x^{\prime}, \mu\right) D^{2} \varphi\left(x^{\prime}\right)\right)+b_{1}\left(x, x^{\prime}, \mu\right) D \varphi\left(x^{\prime}\right), x, x^{\prime} \in \mathbb{R}^{d},
$$

defined for probability measures $\mu$ on $\mathbb{R}^{d}$ and functions $\varphi \in C_{K}^{2}\left(\mathbb{R}^{d}\right)$, and by $L(x, \mu)^{*}$ we denote its dual operator applying to the probability measures on $\mathbb{R}^{d}$. It is well known that $\mu=\left(\mu_{s}\right)_{s \in[t, T])}$ can be characterized as the weak solution of the PDE with stochastic coefficients

$$
\frac{d}{d s} \mu_{s}=L\left[\mu_{s}, \bar{X}_{s}^{0}\right]^{*} \mu_{s}, s \in(t, T], \mu_{t}(d y)=\delta_{\bar{x}}(d y),
$$

i.e.,

$$
\frac{d}{d s}\left\langle\mu_{s}, \varphi\right\rangle=\left\langle\mu_{s}, L\left[\mu_{s}, \bar{X}_{s}^{0}\right] \varphi\right\rangle=\left\langle L\left[\mu_{s}, \bar{X}_{s}^{0}\right]^{*} \mu_{s}, \varphi\right\rangle, \text { for all } \varphi \in C_{K}^{2}\left(\mathbb{R}^{d}\right)
$$

$\left(C_{K}^{2}\left(\mathbb{R}^{d}\right)\right.$ denotes the space of $C^{2}$-functions with compact support in $\left.\mathbb{R}^{d}\right)$.

Let us first remark the following proposition.

Proposition 2.2. Under our standard assumptions the above system (2.5) has a unique solution $\bar{X}^{j}=$ $\left(\bar{X}_{s}^{j}\right)_{s \in[t, T]} \in \mathcal{S}_{\mathbb{F}}^{2}\left(t, T ; \mathbb{R}^{d}\right), j \geq 0$. Moreover, (2.5) can be equivalently rewritten in the following form:

$$
\begin{array}{lll}
d \bar{X}_{s}^{0}=E\left[b_{0}\left(\bar{X}_{s}^{0}, \bar{X}_{s}^{\ell_{0}}\right) \mid \mathcal{F}_{T}^{W^{0}}\right] d s+E\left[\sigma_{0}\left(\bar{X}_{s}^{0}, \bar{X}_{s}^{\ell_{0}}\right) \mid \mathcal{F}_{T}^{W^{0}}\right] d W_{s}^{0}, & \bar{X}_{t}^{0}=x_{0}, \\
d \bar{X}_{s}^{j}=E\left[b_{1}\left(\bar{X}_{s}^{0}, \bar{X}_{s}^{j}, \bar{X}_{s}^{\ell_{j}}\right) \mid \mathcal{F}_{T}^{W^{0}, W^{j}}\right] d s+E\left[\sigma_{1}\left(\bar{X}_{s}^{0}, \bar{X}_{s}^{j}, \bar{X}_{s}^{\ell_{j}}\right) \mid \mathcal{F}_{T}^{W^{0}, W^{j}}\right] d W_{s}^{j}, & \bar{X}_{t}^{j}=\bar{x}, j \geq 1,
\end{array}
$$

where $s$ runs the time interval $[t, T)$ and $\ell_{j} \geq 1$ is arbitrary but different from $j, j \geq 0$.

The equivalence between the first equation of (2.5) and that of (2.10) is evident, since we can replace $\bar{X}^{1}$ in (2.6) by $\bar{X}^{\ell_{1}}$ without changing $\mu_{s}$. The equivalence between the second equation of (2.5) and that of (2.10) follows from the fact that $\bar{X}^{\ell_{j}}$ defined by (2.10) is $\mathbb{F}^{W^{0}, W^{\ell_{j}}}$-adapted and, knowing $\mathcal{F}_{T}^{W^{0}}$ its law doesn't depend on $\ell_{j} \geq 1$. Hence, since, for $\ell_{j} \geq 1$ different from $j, W^{0}, W^{j}$ and $W^{\ell_{j}}$ are independent,

$$
P\left\{\bar{X}_{s}^{\ell_{j}} \in d y \mid \mathcal{F}_{T}^{W^{0}, W^{j}}\right\}=P\left\{\bar{X}_{s}^{\ell_{j}} \in d y \mid \mathcal{F}_{T}^{W^{0}}\right\}=\mu_{s}(d y) .
$$

Proof. The proof is a direct consequence of the above observation. Indeed, we have

$$
\begin{array}{rlrl}
d \bar{X}_{s}^{0} & =E\left[b_{0}\left(\bar{X}_{s}^{0}, \bar{X}_{s}^{1}\right) \mid \mathcal{F}_{T}^{W^{0}}\right] d s+E\left[\sigma_{0}\left(\bar{X}_{s}^{0}, \bar{X}_{s}^{1}\right) \mid \mathcal{F}_{T}^{W^{0}}\right] d W_{s}^{0}, & & \bar{X}_{t}^{0}=x_{0}, \\
d \bar{X}_{s}^{1} & =E\left[b_{1}\left(\bar{X}_{s}^{0}, \bar{X}_{s}^{1}, \bar{X}_{s}^{2}\right) \mid \mathcal{F}_{T}^{W^{0}, W^{1}}\right] d s+E\left[\sigma_{1}\left(\bar{X}_{s}^{0}, \bar{X}_{s}^{1}, \bar{X}_{s}^{2}\right) \mid \mathcal{F}_{T}^{W^{0}, W^{1}}\right] d W_{s}^{1}, & \bar{X}_{t}^{1}=\bar{x}, \\
d \bar{X}_{s}^{2} & =E\left[b_{1}\left(\bar{X}_{s}^{0}, \bar{X}_{s}^{2}, \bar{X}_{s}^{1}\right) \mid \mathcal{F}_{T}^{W^{0}, W^{2}}\right] d s+E\left[\sigma_{1}\left(\bar{X}_{s}^{0}, \bar{X}_{s}^{2}, \bar{X}_{s}^{1}\right) \mid \mathcal{F}_{T}^{W^{0}, W^{2}}\right] d W_{s}^{2}, & \bar{X}_{t}^{2}=\bar{x}
\end{array}
$$

it's a finite-dimensional SDE with Lipschitz coefficients, and standard estimates to show the existence and the uniqueness. Once having the processes $\bar{X}^{0}, \bar{X}^{1}$ and $\bar{X}^{2}$, we can obtain the unique solution processes $\bar{X}^{j}, j \geq 3$, in (2.10) by choosing, for instance, $\ell_{j}=1$. 
The limit system (2.5), or equivalently (2.10), is related with (2.1) and (2.2) through the following convergence property.

Proposition 2.3. Under our standard assumptions we have that, for all $m \geq 1$, there is some constant $C_{m} \in \mathbb{R}_{+}$such that, for all $N \geq 0,0 \leq \ell \leq N$,

$$
E\left[\sup _{r \in[t, T]}\left(\left|X_{r}^{\ell, N}-\bar{X}_{r}^{\ell}\right|^{2}+\frac{1}{N} \sum_{\ell=1}^{N}\left|X_{r}^{\ell, N}-\bar{X}_{r}^{\ell}\right|^{2}\right)^{m}\right] \leq \frac{C_{m}}{N^{m}}+C_{m}\left(\frac{1}{N} \sum_{\ell=1}^{N}\left|x_{\ell}-\bar{x}\right|^{2}\right)^{m} .
$$

For the reader's convenience we give the proof in Appendix 1. By adapting the argument of the proof of the above proposition we also see the following lemma.

Lemma 2.1. Under our standard assumptions we have, for all bounded Lipschitz functions $h: \mathbb{R}^{d} \times \mathbb{R}^{d} \rightarrow \mathbb{R}$ and $g: \mathbb{R}^{d} \rightarrow \mathbb{R}$, and for all $m \geq 1$, the existence of a real constant $C_{m}$ such that, for all $N \geq 1$,

$$
\sup _{s \in[t, T]} E\left[\left|\frac{1}{N} \sum_{\ell=1}^{N} h\left(X_{s}^{0, N}, X_{s}^{\ell, N}\right)-E\left[h\left(\bar{X}_{s}^{0}, \bar{X}_{s}^{1}\right) \mid \mathcal{F}_{T}^{W^{0}}\right]\right|^{2 m}\right] \leq C_{m}\left(\frac{1}{N}+\frac{1}{N} \sum_{\ell=1}^{N}\left|x_{\ell}-\bar{x}\right|^{2}\right)^{m},
$$

and

$$
E\left[\left|\frac{1}{N} \sum_{\ell=1}^{N} g\left(X_{s}^{\ell, N}\right)-E\left[g\left(\bar{X}_{s}^{1}\right) \mid \mathcal{F}_{T}^{W^{0}}\right]\right|^{2 m} \mid \mathcal{F}_{T}^{W^{0}}\right] \leq C_{m}\left(\frac{1}{N}+\frac{1}{N} \sum_{\ell=1}^{N}\left|x_{\ell}-\bar{x}\right|^{2}\right)^{m}, \quad s \in[t, T] .
$$

After having reviewed the limit behavior of the forward equation (2.1)-(2.2) let us come now to the backward one.

\subsection{Limit behavior of the backward stochastic differential equations}

In this subsection we discuss briefly the limit behavior of the solution $\left(Y^{N}, Z^{N}=\left(Z^{j, N}\right)_{0 \leq j \leq N}\right) \in$ $\mathcal{S}_{\mathbb{F}}^{2}(t, T) \times L_{\mathbb{F}}^{2}\left(t, T ; \mathbb{R}^{d}\right)^{N+1}$ of $\mathrm{BSDE}$

$$
\begin{aligned}
& d Y_{s}^{N}=-\frac{1}{N} \sum_{\ell=1}^{N} f\left(X_{s}^{0, N}, X_{s}^{\ell, N}, Y_{s}^{N}, Z_{s}^{0, N}, Z_{s}^{\ell, N}\right) d s+\sum_{j=0}^{N} Z_{s}^{j, N} d W_{s}^{j}, \quad s \in[t, T], \\
& Y_{T}^{N}=\frac{1}{N} \sum_{\ell=1}^{N} \Phi\left(X_{T}^{0, N}, X_{T}^{\ell, N}\right),
\end{aligned}
$$

as $N$ tends to infinity, and we show $\left(Y^{N}, Z^{0, N}\right)$ converges to the unique solution $\left(\bar{Y}, \bar{Z}^{0}\right) \in \mathcal{S}_{\mathbb{F}^{0}}^{2}(t, T) \times$ $L_{\mathbb{F}^{W^{0}}}^{2}\left(t, T ; \mathbb{R}^{d}\right)$ of the BSDE:

$$
\begin{aligned}
& d \bar{Y}_{s}=-f\left(\bar{X}_{s}^{0}, \mu_{s}, \bar{Y}_{s}, \bar{Z}_{s}^{0}, 0\right) d s+\bar{Z}_{s}^{0} d W_{s}^{0}, \quad s \in[t, T], \\
& \bar{Y}_{T}=\Phi\left(\bar{X}_{T}^{0}, \mu_{T}\right)
\end{aligned}
$$

and $Z^{\ell, N}$ converges to 0 , for every $\ell \geq 1$ (recall that $\mu_{s}(d y)=P\left\{\bar{X}_{s}^{1} \in d y \mid \mathcal{F}_{T}^{W^{0}}\right\}$ ). We refer to the fact that it is by now standard that under our assumptions on the coefficients the BSDEs (2.15) and (2.16) have a unique solution. In analogy to the forward system we also see that the limit BSDE (2.16) can be equivalently written in the form

$$
\bar{Y}_{s}=E\left[\Phi\left(\bar{X}_{T}^{0}, \bar{X}_{T}^{\ell}\right) \mid \mathcal{F}_{T}^{W^{0}}\right]+\int_{s}^{T} E\left[f\left(\bar{X}_{r}^{0}, \bar{X}_{r}^{\ell}, \bar{Y}_{r}, \bar{Z}_{r}^{0}, 0\right) \mid \mathcal{F}_{T}^{W^{0}}\right] d r-\int_{s}^{T} \bar{Z}_{r}^{0} d W_{r}^{0}, s \in[t, T],
$$

for any $\ell \geq 1$. Moreover, we can have the following statement on convergence:

Proposition 2.4. Under our standard assumptions on the coefficients we have for all $m \geq 1$ the existence of a constant $C_{m}$ such that

$$
\begin{aligned}
& E\left[\sup _{s \in[t, T]}\left|Y_{s}^{N}-\bar{Y}_{s}\right|^{2 m}+\left(\int_{t}^{T}\left|Z_{s}^{0, N}-\bar{Z}_{s}^{0}\right|^{2} d s+\sum_{\ell=1}^{N} \int_{t}^{T}\left|Z_{s}^{\ell, N}\right|^{2} d r\right)^{m}\right] \\
& \leq C_{m}\left(\frac{1}{N}+\frac{1}{N} \sum_{\ell=1}^{N}\left|x_{\ell}-\bar{x}\right|^{2}\right)^{m}, \text { for all } N \geq 1 .
\end{aligned}
$$

For the reader's convenience the proof is given in the Appendix 2 . 


\section{The stochastic differential game with $\mathrm{N}+1$ participants}

Let $U=\mathbb{R}^{k}$ be the control state space for the major player and $V=\mathbb{R}^{m}$ for the minor agents. To simplify the notation, we will suppose from now on that the dimension $d$ used in the preceding section is equal to 1.

Our objective is to study the limit behavior of the stochastic differential game between a major player and $N$ collectively behaving minor agents, when $N \rightarrow+\infty$.

We denote by $\mathbb{F}^{N}$ the filtration generated by the Brownian motion $W^{(N)}=\left(W^{0}, W^{1}, \ldots, W^{N}\right)$ and augmented by all $P$-null sets, and by $L_{N}^{\infty}\left(t, T ; \mathbb{R}^{\ell}\right)(\ell \geq 1)$ we denote the space of all bounded, measurable functionals $\gamma:[t, T] \times C([t, T]) \rightarrow \mathbb{R}^{\ell}$ which are non-anticipating, i.e., for all $s \in[t, T]$ and all $\psi, \psi^{\prime} \in C([t, T])$ with $\psi(r)=\psi^{\prime}(r), r \in[t, s]$, it holds $\gamma_{r}(\psi)=\gamma_{r}\left(\psi^{\prime}\right), r \in[t, s]$. Given initial positions $x^{(N)}=\left(x_{0}, x_{1}, \ldots, x_{N}\right)$, a feedback control $u \in L_{N}^{\infty}(t, T ; U)$ of the major player, and feedback controls $v^{j} \in L_{N}^{\infty}(t, T ; V), 1 \leq j \leq N$, for the $N$ minor agents, the dynamics of the major player $X^{0, N}$ and those of the minor agents $X^{\ell, N}$ are defined by the system

$$
\begin{aligned}
& d X_{s}^{0, N}=\sigma_{0}\left(X_{s}^{0, N}\right) d \tilde{W}_{s}^{0}+\frac{1}{N} \sum_{\ell=1}^{N} b_{0}\left(X_{s}^{0, N}, X_{s}^{\ell, N}, Z_{s}^{0, N}\right) u_{s}\left(X^{(N)}\right) d s, \quad X_{t}^{0, N}=x_{0}, \\
& d X_{s}^{j, N}=\sigma_{1}\left(X_{s}^{j, N}\right) d \tilde{W}_{s}^{j}+\frac{\varepsilon_{N}}{N} \sum_{\ell=1}^{N} b_{1}\left(X_{s}^{0, N}, X_{s}^{\ell, N}, Z_{s}^{j, N}\right) v_{s}^{\ell}\left(X^{(N)}\right) d s, \quad X_{t}^{j, N}=x_{j},
\end{aligned}
$$

$1 \leq j \leq N$, driven by an $N+1$-dimensional Brownian motion $\left(\tilde{W}^{0}, \tilde{W}^{1}, \cdots, \tilde{W}^{N}\right)$. And the nonlinear payoff/cost functional is defined through the BSDE

$$
\begin{aligned}
d Y_{s}^{N} & =-\frac{1}{N} \sum_{\ell=1}^{N} f\left(X_{s}^{0, N}, X_{s}^{\ell, N}, Y_{s}^{N}, Z_{s}^{0, N}, Z_{s}^{\ell, N},\left(u_{s}, v_{s}^{\ell}\right)\left(X^{(N)}\right)\right) d s+\sum_{\ell=0}^{N} Z_{s}^{\ell, N} d \tilde{W}_{s}^{\ell}, s \in[t, T] \\
Y_{T}^{N} & =\frac{1}{N} \sum_{\ell=1}^{N} \Phi\left(X_{T}^{0, N}, X_{T}^{\ell, N}\right) .
\end{aligned}
$$

Here $X^{(N)}=\left(X^{0, N}, \ldots, X^{N, N}\right)$, and $\varepsilon_{N}>0$ is a factor which converges to zero, as $N$ tends to $\infty$ (its role will be discussed later). We want to study the above system in a weak sense, i.e., we allow the driving $N$-dimensional Brownian motion $\left(\tilde{W}^{0}, \ldots, \tilde{W}^{N}\right)$ to depend on the control processes. Assuming for simplicity that $\sigma_{0} \equiv 1, \sigma_{1} \equiv 1$, we use the Girsanov transformation

$$
\begin{aligned}
d \tilde{W}_{s}^{0} & =d W_{s}^{0}-I_{[t, T]}(s) \frac{1}{N} \sum_{\ell=1}^{N} b_{0}\left(X_{s}^{0, N}, X_{s}^{\ell, N}, Z_{s}^{0, N}\right) u_{s}\left(X_{.}^{(N)}\right) d s, \\
d \tilde{W}_{s}^{j} & =d W_{s}^{j}-I_{[t, T]}(s) \frac{\varepsilon_{N}}{N} \sum_{\ell=1}^{N} b_{1}\left(X_{s}^{0, N}, X_{s}^{\ell, N}, Z_{s}^{j, N}\right) v_{s}^{\ell}\left(X^{(N)}\right) d s,
\end{aligned}
$$

in order to reduce the study of the above system to the resolution of the following simplified system:

$$
\begin{aligned}
X_{s}^{j, N}= & X_{s}^{j}=x_{j}+W_{s}^{j}-W_{t}^{j}, 0 \leq j \leq N, \\
d Y_{s}^{N}= & -\left\{\frac{1}{N} \sum_{\ell=1}^{N} f\left(X_{s}^{0, N}, X_{s}^{\ell, N}, Y_{s}^{N}, Z_{s}^{0, N}, Z_{s}^{\ell, N}, u_{s}, v_{s}^{\ell}\right)+\left(\frac{1}{N} \sum_{\ell=1}^{N} b_{0}\left(X_{s}^{0, N}, X_{s}^{\ell, N}, Z_{s}^{0, N}\right) u_{s}\right) Z_{s}^{0, N}\right. \\
& \left.+\sum_{j=1}^{N}\left(\frac{\varepsilon_{N}}{N} \sum_{\ell=1}^{N} b_{1}\left(X_{s}^{0, N}, X_{s}^{\ell, N}, Z_{s}^{j, N}\right) v_{s}^{\ell}\right) Z_{s}^{j, N}\right\} d s+\sum_{\ell=0}^{N} Z_{s}^{\ell, N} d W_{s}^{\ell}, \quad s \in[t, T], \\
Y_{T}^{N}= & \frac{1}{N} \sum_{\ell=1}^{N} \Phi\left(X_{T}^{0, N}, X_{T}^{\ell, N}\right) .
\end{aligned}
$$

Here $u_{s}:=u_{s}\left(x^{(N)}+W^{(N)}-W_{t}^{(N)}\right), v_{s}^{\ell}:=v_{s}^{\ell}\left(x^{(N)}+W^{(N)}-W_{t}^{(N)}\right), s \in[t, T], 1 \leq \ell \leq N$, are now open-loop controls, and we can work for the system (3.3) with open-loop controls $u \in \mathcal{U}_{N}:=L_{\mathbb{F}^{N}}^{\infty}(t, T ; U)$ and $v^{\ell} \in \mathcal{V}_{N}:=L_{\mathbb{F}^{N}}^{\infty}(t, T ; V), 1 \leq \ell \leq N$, as long as our saddle point controls are feedback controls.

Our objective is to study this latter stochastic differential game and its limit behavior as $N \rightarrow+\infty$. In order to abbreviate the notation, given $\xi^{(N)}:=\left(x^{(N)}=\left(x_{0}, \ldots, x_{N}\right), y, z^{(N)}=\left(z_{0}, \ldots, z_{N}\right)\right) \in \mathbb{R}^{N+1} \times$ $\mathbb{R} \times \mathbb{R}^{N+1}, u \in U$ and $v^{(N)}=\left(v_{1}, \ldots, v_{N}\right) \in V^{N}$, we denote the driving coefficient of the BSDE in (3.3) by

$$
\begin{aligned}
& H_{N}\left(\xi^{(N)}, u, v^{(N)}\right) \\
& =\frac{1}{N} \sum_{\ell=1}^{N} f\left(x_{0}, x_{\ell}, y, z_{0}, z_{\ell}, u, v_{\ell}\right)+\left(\frac{1}{N} \sum_{\ell=1}^{N} b_{0}\left(x_{0}, x_{\ell}, z_{0}\right) u\right) z_{0}+\varepsilon_{N} \sum_{i=1}^{N}\left(\frac{1}{N} \sum_{\ell=1}^{N} b_{1}\left(x_{0}, x_{\ell}, z_{i}\right) v_{\ell}\right) z_{i} .
\end{aligned}
$$

Let us make throughout our paper the following assumptions on the coefficients involved in the definition of the Hamiltonian $H_{N}$ :

Assumptions. 1) $f=f\left(x_{0}, x_{1}, y, z_{0}, z_{1}, u, v\right): \mathbb{R}^{5} \times U \times V \rightarrow \mathbb{R}$ is a continuous function with $f(\xi, .,.) \in$ $C^{1}, \xi:=\left(\eta, z_{1}\right) \in \mathbb{R}^{5}, \eta:=\left(x_{0}, x_{1}, y, z_{0}\right) \in \mathbb{R}^{4}$, such that 
- Ai) For some $\lambda>0$ :

$$
\left\{\begin{array}{l}
\left.\left(D_{u} f\right)(\xi, u, v)-\left(D_{u} f\right)\left(\xi, u^{\prime}, v\right), u-u^{\prime}\right) \leq-\lambda\left|u-u^{\prime}\right|^{2} \\
\left.\left(D_{v} f\right)(\xi, u, v)-\left(D_{v} f\right)\left(\xi, u, v^{\prime}\right), v-v^{\prime}\right) \geq \lambda\left|v-v^{\prime}\right|^{2}
\end{array}\right.
$$

for all $\xi \in \mathbb{R}^{5},(u, v),\left(u^{\prime}, v^{\prime}\right) \in U \times V$;

- Aii) For some constants $C>0$ and $\mu(0<\mu<\lambda)$,

$$
\begin{aligned}
& |f(\xi, 0,0)| \leq C ; \\
& \left|f(\xi, u, v)-f\left(\xi^{\prime}, u, v\right)\right| \leq C\left|\xi-\xi^{\prime}\right| ; \\
& \left|\left(D_{u} f\right)(\xi, u, v)-\left(D_{u} f\right)\left(\xi^{\prime}, u^{\prime}, v^{\prime}\right)\right| \leq C\left(\left|\xi-\xi^{\prime}\right|+\left|u-u^{\prime}\right|\right)+\mu\left|v-v^{\prime}\right|, \quad\left|\left(D_{u} f\right)(\xi, 0,0)\right| \leq C ; \\
& \left|\left(D_{v} f\right)(\xi, u, v)-\left(D_{v} f\right)\left(\xi^{\prime}, u^{\prime}, v^{\prime}\right)\right| \leq C\left(\left|\eta-\eta^{\prime}\right|+\left|v-v^{\prime}\right|\right)+\mu\left|u-u^{\prime}\right|, \quad\left|\left(D_{v} f\right)(\xi, 0,0)\right| \leq C ;
\end{aligned}
$$

2) The coefficients $b_{0}: \mathbb{R}^{3} \rightarrow U$ and $b_{1}: \mathbb{R}^{3} \rightarrow V$ are bounded and continuous, such that $b_{i}\left(x_{0}, x_{1}, z\right) z$ is bounded and Lipschitz in $\left(x_{0}, x_{1}, z\right), i=0,1$.

3) The function $\Phi: \mathbb{R} \rightarrow \mathbb{R}$ is bounded and Lipschitz.

Remark 3.1. 1. From Ai) we see $f(\xi, \cdot, \cdot): U \times V \rightarrow \mathbb{R}$ is a strictly concave-convex function. This strict concavity-convexity will be crucial for the study of saddle point controls for the associated stochastic differential game and the study of their behavior, as $N$ tends to $+\infty$.

An easy example for such a function $f$ satisfying our assumptions Ai) and Aii) is the following one for $k=m=1$ :

$$
f(\xi, u, v)=f_{0}(\xi, u, v)-\frac{1}{2} \alpha|u|^{2}+\frac{1}{2} \gamma|v|^{2},
$$

where $\alpha, \gamma>0$ are strictly positive and $f_{0} \in C_{b}^{1,2,2}\left(\mathbb{R}^{5} \times U \times V\right)$ is such that $D_{u u}^{2} f_{0}(\xi, u, v) \leq(\alpha-\lambda) I_{U}(u)$ and $D_{v v}^{2} f_{0}(\xi, u, v) \geq(\lambda-\gamma) I_{V}(v),(\xi, u, v) \in \mathbb{R}^{5} \times U \times V$, for some $\lambda>0$, and $\left|D_{u v}^{2} f_{0}(\xi, u, v)\right| \leq \mu$, $(\xi, u, v) \in \mathbb{R}^{5} \times U \times V$, for some $\mu<\lambda$.

2. Let us also give an example of a function $b_{i}\left(x_{0}, x_{1}, z\right)$ satisfying Assumptions 2): For this let $\tilde{b}_{i}: \mathbb{R}^{3} \rightarrow \mathbb{R}$ be a bounded Lipschitz function and consider

$$
b_{i}\left(x_{0}, x_{1}, z\right)=\tilde{b}_{i}\left(x_{0}, x_{1}, z\right) \cdot \frac{1}{1+|z|} .
$$

We also observe that from our assumptions on the function $f$ we get

i) $|f(\xi, u, v)| \leq C\left(1+|u|^{2}+|v|^{2}\right)$;

ii) $|f(\xi, u, v)-f(\tilde{\xi}, \tilde{u}, \tilde{v})| \leq C|\xi-\tilde{\xi}|+C(|u|+|\tilde{u}|+|v|+|\tilde{v}|)(|u-\tilde{u}|+|v-\tilde{v}|)$,

for all $\xi=\left(x_{0}, x_{1}, y, z_{0}, z_{1}\right), \tilde{\xi}=\left(\tilde{x}_{0}, \tilde{x}_{1}, \tilde{y}, \tilde{z}_{0}, \tilde{z}_{1}\right) \in \mathbb{R}^{5}$ and $u, \tilde{u} \in U, v, \tilde{v} \in V$. Hence, taking into account the above assumption on the coefficients $b_{0}$ and $b_{1}$, we see that, for all $\xi^{(N)}=\left(x^{(N)}=\left(x_{0}, \ldots, x_{N}\right), y, z^{(N)}=\right.$ $\left.\left(z_{0}, \ldots, z_{N}\right)\right), \tilde{\xi}^{(N)}=\left(\tilde{x}^{(N)}=\left(\tilde{x}_{0}, \ldots, \tilde{x}_{N}\right), \tilde{y}, \tilde{z}^{(N)}=\left(\tilde{z}_{0}, \ldots, \tilde{z}_{N}\right)\right), \xi^{(N, 0)}=\left(x^{(N)}=\left(x_{0}, \ldots, x_{N}\right), y, 0\right) \in$ $\mathbb{R}^{N+1} \times \mathbb{R} \times \mathbb{R}^{N+1}$, and $u \in U, v^{(N)} \in V^{N}$, we have

- $\left|H_{N}\left(\xi^{(N, 0)}, u, v^{(N)}\right)\right| \leq C\left(1+|u|^{2}+\frac{1}{N}\left|v^{(N)}\right|^{2}\right)$;

- $\left|H_{N}\left(\xi^{(N)}, u, v^{(N)}\right)-H_{N}\left(\tilde{\xi}^{(N)}, u, v^{(N)}\right)\right|$

$$
\begin{aligned}
& \leq C\left(1+|u|+\varepsilon_{N} \sum_{\ell=1}^{N}\left|v_{\ell}\right|\right)\left(\left|x_{0}-\tilde{x}_{0}\right|+|y-\tilde{y}|+\left|z_{0}-\tilde{z}_{0}\right|+\frac{1}{N} \sum_{\ell=1}^{N}\left(\left|x_{\ell}-\tilde{x}_{\ell}\right|+\left|z_{\ell}-\tilde{z}_{\ell}\right|\right)\right) \\
& \quad+C \varepsilon_{N} \sum_{\ell=1}^{N}\left|v_{\ell}\right|\left|x_{\ell}-\tilde{x}_{\ell}\right| .
\end{aligned}
$$

The above properties of $H_{N}$ allow to apply the classical result on the existence and uniqueness of the solution for BSDE:

Proposition 3.1. For any admissible controls $u \in \mathcal{U}_{N}=L_{\mathbb{F}^{N}}^{\infty}(t, T ; U)$ and $v^{(N)}=\left(v^{1, N}, \ldots, v^{N, N}\right) \in \mathcal{V}_{N}^{N}=$ $L_{\mathbb{F}^{N}}^{\infty}(t, T ; V)^{N}$, the BSDE in (3.3) admits a unique solution $\left(Y^{N}, Z^{N}=\left(Z^{0, N}, \ldots, Z^{N, N}\right)\right) \in \mathcal{S}_{\mathbb{F}^{N}}^{2}(t, T) \times$ $L_{\mathbb{F}^{N}}^{2}(t, T)^{N+1}$. In order to indicate that this solution is associated with the controls $\left(u, v^{(N)}\right)$, we also write

$$
\left(Y^{u, v^{(N)}}, Z^{u, v^{(N)}}=\left(Z^{0, u, v^{(N)}}, \ldots, Z^{N, u, v^{(N)}}\right)\right):=\left(Y^{N}, Z^{N}=\left(Z^{0, N}, \ldots, Z^{N, N}\right)\right) .
$$


As already explained, we have two objectives: First we want to study for each fixed $N \geq 1$ saddle point controls for our stochastic differential game with $N$ collectively acting minor agents playing against one major player, i.e, we are looking for a couple of controls $\left(\bar{u}^{N}, \bar{v}^{(N)}=\left(\bar{v}^{1, N}, \ldots, \bar{v}^{N, N}\right)\right) \in \mathcal{U}_{N} \times \mathcal{V}_{N}^{N}$ such that

$$
Y_{t}^{u, \bar{v}^{(N)}} \leq Y_{t}^{\bar{u}^{N}, \bar{v}^{(N)}} \leq Y_{t}^{\bar{u}^{N}, v^{(N)}}, \text { for all }\left(u, v^{(N)}\right) \in \mathcal{U}_{N} \times \mathcal{V}_{N}^{N} .
$$

In a second step we are interested in the limit behavior of the saddle point controls $\left(\bar{u}^{N}, \bar{v}^{(N)}\right)$ when $N$ tends to $+\infty$, and we want to characterize the limit controls as saddle point controls for the limit stochastic differential game.

Let us begin with the study of the existence of saddle point controls and its characterization for the game with $N+1$ participants. For this end, we have first to point out some useful properties of the Hamiltonian $H_{N}$. So we observe that, as a direct consequence of the above assumptions on our coefficients, we have

$$
\begin{aligned}
& \left(\left(D_{u} H_{N}\right)\left(\xi^{(N)}, u, v^{(N)}\right)-\left(D_{u} H_{N}\right)\left(\xi^{(N)}, \tilde{u}, v^{(N)}\right), u-\tilde{u}\right) \leq-\lambda|u-\tilde{u}|^{2} \\
& \left(\left(D_{v^{(N)}} H_{N}\right)\left(\xi^{(N)}, u, v^{(N)}\right)-\left(D_{v^{(N)}} H_{N}\right)\left(\xi^{(N)}, u, \tilde{v}^{(N)}\right), v^{(N)}-\tilde{v}^{(N)}\right) \\
& \quad \geq \frac{\lambda}{N} \sum_{l=1}^{N}\left|v_{l}-\tilde{v}_{l}\right|^{2}=\frac{\lambda}{N}\left|v^{(N)}-\tilde{v}^{(N)}\right|^{2} .
\end{aligned}
$$

From (3.8) it follows immediately that, for all $\xi^{(N)} \in \mathbb{R}^{N+1} \times \mathbb{R} \times \mathbb{R}^{N+1}$, the function $H_{N}\left(\xi^{(N)}, \cdot, \cdot\right)$ : $U \times V^{N} \rightarrow \mathbb{R}$ has a unique saddle point. More precisely, there exists a couple of Borel measurable feedback controls $\left(\bar{u}^{N}, \bar{v}^{(N)}\right): \mathbb{R}^{N+1} \times \mathbb{R} \times \mathbb{R}^{N+1} \rightarrow U \times V^{N}$, such that for all $\left(u, v^{(N)}\right) \in U \times V^{N}$,

$$
H_{N}\left(\xi^{(N)}, u, \bar{v}^{(N)}\left(\xi^{(N)}\right)\right) \leq H_{N}\left(\xi^{(N)}, \bar{u}^{N}\left(\xi^{(N)}\right), \bar{v}^{(N)}\left(\xi^{(N)}\right)\right) \leq H_{N}\left(\xi^{(N)}, \bar{u}^{N}\left(\xi^{(N)}\right), v^{(N)}\right) .
$$

However, the special form of our Hamiltonian $H_{N}$ allows to compute the form of this saddle point in a more precise manner. For this end, we let $\tilde{v}^{(N)}=\left(\tilde{v}_{1}^{N}, \cdots, \tilde{v}_{N}^{N}\right): \mathbb{R}^{N+1} \times \mathbb{R} \times \mathbb{R}^{N+1} \times U \rightarrow V^{N}$ be a Borel measurable function such that

$$
\begin{aligned}
& H_{N}\left(\xi^{(N)}, u, \tilde{v}^{(N)}\left(\xi^{(N)}, u\right)\right)=\inf _{v^{(N)} \in V^{N}} H_{N}\left(\xi^{(N)}, u, v^{(N)}\right) \\
& =\frac{1}{N} \sum_{l=1}^{N} \inf _{v_{l} \in V}\left\{f\left(x_{0}, x_{l}, y, z_{0}, z_{l}, u, v_{l}\right)+\varepsilon_{N} \sum_{i=1}^{N} b_{1}\left(x_{0}, x_{l}, z_{i}\right) z_{i} v_{l}\right\}+\frac{1}{N}\left(\sum_{l=1}^{N} b_{0}\left(x_{0}, x_{l}, z_{0}\right) z_{0}\right) u,
\end{aligned}
$$

and $\tilde{v}_{N}: \mathbb{R} \times \mathbb{R} \times \mathbb{R} \times \mathbb{R}^{N+1} \times U \rightarrow V$ a measurable function with

$$
\begin{aligned}
& f\left(\xi_{\ell}, u, \tilde{v}_{N}\left(\xi_{\ell}^{(N)}, u\right)\right)+\varepsilon_{N} \sum_{i=1}^{N} b_{1}\left(x_{0}, x_{\ell}, z_{i}\right) z_{i} \tilde{v}_{N}\left(\xi_{\ell}^{(N)}, u\right) \\
& =\inf _{v_{l} \in V}\left(f\left(\xi_{\ell}, u, v_{\ell}\right)+\varepsilon_{N} \sum_{i=1}^{N} b_{1}\left(x_{0}, x_{l}, z_{i}\right) z_{i} v_{\ell}\right)
\end{aligned}
$$

for all $\xi_{\ell}^{(N)}=\left(x_{0}, x_{\ell}, y, z^{(N)}\right)$ and all $u \in U$. Obviously,

$$
\tilde{v}_{\ell}^{N}\left(\xi^{(N)}, u\right)=\tilde{v}_{N}\left(\xi_{\ell}^{(N)}, u\right), 1 \leq \ell \leq N .
$$

Let us also consider a measurable function $\tilde{u}^{N}: \mathbb{R}^{N+1} \times \mathbb{R} \times \mathbb{R}^{N+1} \longrightarrow U$ such that

$$
\begin{aligned}
& H_{N}\left(\xi^{(N)}, \tilde{u}^{N}\left(\xi^{(N)}\right), \tilde{v}^{(N)}\left(\xi^{(N)}, \tilde{u}^{N}\left(\xi^{(N)}\right)\right)\right) \\
& =\sup _{u \in U} H_{N}\left(\xi^{(N)}, u, \tilde{v}^{(N)}\left(\xi^{(N)}, u\right)\right)\left(=\sup _{u \in U} \inf _{v^{(N)} \in V^{N}} H_{N}\left(\xi^{(N)}, u, v^{(N)}\right)\right),
\end{aligned}
$$

for all $\xi^{(N)} \in \mathbb{R}^{N+1} \times \mathbb{R} \times \mathbb{R}^{N+1}$.

For the above introduced functions we have the following result: 
Lemma 3.1. Under our standard assumptions the unique saddle point $\left(\bar{u}^{N}\left(\xi^{(N)}\right), \bar{v}^{(N)}\left(\xi^{(N)}\right)\right)$ of $H_{N}\left(\xi^{(N)}, \cdot, \cdot\right)$ is of the form

$$
\left(\bar{u}^{N}\left(\xi^{(N)}\right), \bar{v}^{(N)}\left(\xi^{(N)}\right)\right)=\left(\tilde{u}^{N}\left(\xi^{(N)}\right), \tilde{v}^{(N)}\left(\xi^{(N)}, \tilde{u}^{N}\left(\xi^{(N)}\right)\right)\right), \xi^{(N)} \in \mathbb{R}^{N+1} \times \mathbb{R} \times \mathbb{R}^{N+1},
$$

i.e., it corresponds to a Stackelberg feedback strategy for a 2-player zero-sum game with the major player as leader and the collectively acting minor agents as followers; it is the optimal feedback strategy for the major player, if the collectively acting minor agents react in an optimal way.

For the convenience of the reader we give the proof in Appendix 3.

With the help of our standard assumptions and Lemma 3.1 we can now derive the following estimates for our saddle point controls:

Lemma 3.2. There exists some constant $C \in \mathbb{R}$ such that, for all $N \geq 1, \xi^{(N)}=\left(x^{(N)}, y, z^{(N)}\right), \tilde{\xi}^{(N)}=$ $\left(\tilde{x}^{(N)}, \tilde{y}, \tilde{z}^{(N)}\right) \in \mathbb{R}^{N+1} \times \mathbb{R} \times \mathbb{R}^{N+1}$,

(i) $\left|\bar{u}^{N}\left(\xi^{(N)}\right)\right|$

(ii) $\left|\bar{v}_{\ell}^{N}\left(\xi^{(N)}\right)\right|\left(=\left|\tilde{v}_{N}\left(\xi_{\ell}^{(N)}, \tilde{u}^{N}\left(\xi^{(N)}\right)\right)\right|\right) \leq \min \left\{C\left(1+\varepsilon_{N} \sum_{i=1}^{N}\left|z_{i}\right|\right), C\left(1+\varepsilon_{N} N\right)\right\}, \quad 1 \leq \ell \leq N$.

And

$$
\begin{aligned}
(\mathrm{iii})\left|\bar{u}^{N}\left(\xi^{(N)}\right)-\bar{u}^{N}\left(\tilde{\xi}^{(N)}\right)\right| \leq & C\left(\left|x_{0}-\tilde{x}_{0}\right|+|y-\tilde{y}|+\left|z_{0}-\tilde{z}_{0}\right|\right) \\
& +C\left(1+\varepsilon_{N} N\right)\left(\left|x_{0}-\tilde{x}_{0}\right|+\frac{1}{N} \sum_{\ell=1}^{N}\left|x_{\ell}-\tilde{x}_{\ell}\right|+\frac{1}{N} \sum_{\ell=1}^{N}\left|z_{\ell}-\tilde{z}_{\ell}\right|\right) ; \\
\text { (iv) }\left|\bar{v}_{\ell}^{N}\left(\xi^{(N)}\right)-\bar{v}_{\ell}^{N}\left(\tilde{\xi}^{(N)}\right)\right| \leq & C\left(\left|x_{0}-\tilde{x}_{0}\right|+|y-\tilde{y}|+\left|z_{0}-\tilde{z}_{0}\right|\right) \\
& +C\left(1+\varepsilon_{N} N\right)\left(\left|x_{0}-\tilde{x}_{0}\right|+\left|x_{\ell}-\tilde{x}_{\ell}\right|+\frac{1}{N} \sum_{i=1}^{N}\left(\left|x_{i}-\tilde{x}_{i}\right|+\left|z_{i}-\tilde{z}_{i}\right|\right)\right) .
\end{aligned}
$$

For the proof of this lemma the reader is referred to Appendix 3.

With the help of the couple of feedback saddle point controls $\left(\bar{u}^{N}, \bar{v}^{(N)}=\left(\bar{v}_{1}^{N}, \ldots, \bar{v}_{N}^{N}\right)\right)$ we now introduce the function

$$
\bar{H}_{N}\left(\xi^{(N)}\right):=H_{N}\left(\xi^{(N)}, \bar{u}^{N}\left(\xi^{(N)}\right), \bar{v}^{(N)}\left(\xi^{(N)}\right)\right), \xi^{(N)} \in \mathbb{R}^{N+1} \times \mathbb{R} \times \mathbb{R}^{N+1},
$$

which has the following properties:

Lemma 3.3. 1) Using the notation $\xi^{(N, 0)}=\left(x^{(N)}, y,\left(z_{0}, 0, \cdots, 0\right)\right) \in \mathbb{R}^{N+1} \times \mathbb{R} \times \mathbb{R}^{N+1}$, we have, for some constant $C \in \mathbb{R}$ independent of $N \geq 1$,

$$
\left|\bar{H}_{N}\left(\xi^{(N, 0)}\right)\right| \leq C \text {. }
$$

2) There is a constant $C_{N}$ (depending on $N \geq 1$ ) such that, for all $\xi^{(N)}, \tilde{\xi}^{(N)} \in \mathbb{R}^{N+1} \times \mathbb{R} \times \mathbb{R}^{N+1}$,

$$
\left|\bar{H}_{N}\left(\xi^{(N)}\right)-\bar{H}_{N}\left(\tilde{\xi}^{(N)}\right)\right| \leq C_{N}\left|\xi^{(N)}-\tilde{\xi}^{(N)}\right| .
$$

The proof of this lemma follows immediately from the assumptions on $b_{0}$ and $b_{1}$, and our estimates (3.6) for $f$, combined with the estimates given in Lemma 3.2. Consequently, we can have the following result:

Lemma 3.4. Under our assumptions, there exists a unique solution $\left(\bar{Y}^{N}, \bar{Z}^{(N)}=\left(\bar{Z}^{0, N}, \ldots, \bar{Z}^{N, N}\right)\right) \in$ $\mathcal{S}_{\mathbb{F}^{N}}^{2}(t, T) \times L_{\mathbb{F}^{N}}^{2}(t, T)^{N+1}$ of the $B S D E$

$$
\begin{aligned}
\mathrm{d} \bar{Y}_{s}^{N} & =-\bar{H}_{N}\left(X_{s}^{(N)}, \bar{Y}_{s}^{N}, \bar{Z}_{s}^{(N)}\right) \mathrm{d} s+\sum_{j=0}^{N} \bar{Z}_{s}^{j, N} \mathrm{~d} W_{s}^{j}, \quad s \in[t, T], \\
\bar{Y}_{T}^{N} & =\frac{1}{N} \sum_{\ell=1}^{N} \Phi\left(X_{T}^{0}, X_{T}^{\ell, N}\right) .
\end{aligned}
$$

By putting

$$
\bar{u}_{s}^{N}:=\bar{u}^{N}\left(X_{s}^{(N)}, \bar{Y}_{s}^{N}, \bar{Z}_{s}^{(N)}\right), \quad \bar{v}_{s}^{(N)}:=\bar{v}^{(N)}\left(X_{s}^{(N)}, \bar{Y}_{s}^{N}, \bar{Z}_{s}^{(N)}\right), s \in[t, T],
$$


and $\bar{u}_{s}^{N}:=u, \bar{v}_{s}^{(N)}:=v^{(N)}, s \in[0, t)$, for arbitrarily chosen $u \in U$ and $v^{(N)} \in V^{N}$, we define admissible control processes $\bar{u}^{N} \in \mathcal{U}_{N}, \bar{v}^{(N)}=\left(\bar{v}^{(1, N)}, \ldots, \bar{v}^{(N, N)}\right) \in \mathcal{V}_{N}^{N}$ (abusing notation we use nearly the same notations as for the corresponding feedback controls). The following statement is now a standard statement following from the uniqueness of the solution of BSDE in (3.3), from (3.9) and the comparison theorem for BSDEs.

Proposition 3.2. The above introduced couple of admissible controls $\left(\bar{u}^{N}, \bar{v}^{(N)}\right) \in \mathcal{U}_{N} \times \mathcal{V}_{N}^{N}$ forms a saddle point for the pay-off/cost functional $\mathcal{U}_{N} \times \mathcal{V}_{N}^{N} \ni\left(u, v^{(N)}\right) \rightarrow Y_{t}^{u, v^{(N)}}$. More precisely, we even have

$$
Y_{s}^{u, \bar{v}^{(N)}} \leq Y_{s}^{\bar{u}^{N}, \bar{v}^{(N)}} \leq Y_{s}^{\bar{u}^{N}, v^{(N)}}, s \in[t, T]
$$

P-a.s., for all $\left(u, v^{(N)}\right) \in \mathcal{U}_{N} \times \mathcal{V}_{N}^{N}$. Moreover,

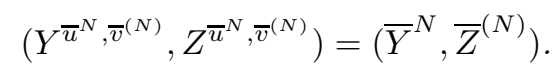

\section{The limit game}

\subsection{The limit backward stochastic differential equation}

After having shown in the preceding subsection the existence of saddle point controls $\left(\bar{u}^{N}, \bar{v}^{(N)}\right) \in$ $\mathcal{U}_{N} \times \mathcal{V}_{N}^{N}$ for the game with $N+1$ agents, the objective of this subsection is to introduce a BSDE, for which we will prove later that it is the limit of the BSDEs for $N+1$ participants, if the saddle point controls are played, and we will study the associated saddle point control processes for this limit BSDE.

Let us begin with the introduction of the Hamiltonian for our limit BSDE. For this end, we observe that Assumption Ai) allows to select a measurable function $\bar{v}: \mathbb{R}^{4} \times U \rightarrow V$ such that

$$
f(\xi, u, \bar{v}(\xi, u))=\inf _{v \in V} f(\xi, u, v),\left(\xi=\left(x_{0}, x_{1}, y, z_{0}, 0\right), u\right) \in \mathbb{R}^{5} \times U .
$$

To shorten the notation, we identify in what follows $\xi=\left(x_{0}, x_{1}, y, z_{0}, 0\right)$ with $\left(x_{0}, x_{1}, y, z_{0}\right)$ and we put $f_{\bar{v}}(\xi, u):=f(\xi, u, \bar{v}(\xi, u))$. From a straight-forward computation using the assumptions Ai) and Aii) on $f$, we obtain

Lemma 4.1. Under our standard assumptions on $f$, we have:

1. There is some $C \in \mathbb{R}$ such that, for all $(\xi, u),\left(\xi^{\prime}, u^{\prime}\right) \in \mathbb{R}^{4} \times U$,

$$
\begin{aligned}
& |\bar{v}(\xi, u)| \leq C+\frac{\mu}{\lambda}|u| ; \\
& \left|\bar{v}(\xi, u)-\bar{v}\left(\xi^{\prime}, u^{\prime}\right)\right| \leq C\left|\xi-\xi^{\prime}\right|+\frac{\mu}{\lambda}\left|u-u^{\prime}\right| .
\end{aligned}
$$

2. For all $\xi \in \mathbb{R}^{4}, f_{\bar{v}}(\xi,.) \in C^{1}$ and

$$
\left(D_{u} f_{\bar{v}}\right)(\xi, u)=\left(D_{u} f\right)(\xi, u, \bar{v}(\xi, u)),(\xi, u) \in \mathbb{R}^{4} \times U .
$$

With the help of the function $f_{\bar{v}}$ we introduce the Hamiltonian

$$
\begin{aligned}
\bar{H}\left(s, x_{0}, y, z_{0}, u\right): & =E\left[f_{\bar{v}}\left(x_{0}, \bar{X}_{s}^{1}, y, z_{0}, 0, u\right)+b_{0}\left(x_{0}, \bar{X}_{s}^{1}, z_{0}\right) z_{0} \cdot u \mid \mathcal{F}_{T}^{W^{0}}\right] \\
& =E\left[f_{\bar{v}}\left(x_{0}, \bar{X}_{s}^{1}, y, z_{0}, 0, u\right) \mid \mathcal{F}_{T}^{W^{0}}\right]+E\left[b_{0}\left(x_{0}, \bar{X}_{s}^{1}, z_{0}\right) z_{0} \mid \mathcal{F}_{T}^{W^{0}}\right] \cdot u
\end{aligned}
$$

We observe that $\bar{H}\left(s, x_{0}, y, z_{0}, u\right)$ is a continuous random field which, for every fixed $\left(x_{0}, y, z_{0}, u\right)$, is $\mathbb{F}^{W^{0}}$ progressively measurable. Moreover, we have the following further properties of $\bar{H}$ :

Lemma 4.2. 1) There exists some constant $C \in \mathbb{R}$ such that, $P$-a.s., for all $\xi, \xi^{\prime} \in \mathbb{R}^{3}, u, u^{\prime} \in U$,

i) $|\bar{H}(s, \xi, u)| \leq C\left(1+|u|^{2}\right)$;

ii) $\left|\bar{H}(s, \xi, u)-\bar{H}\left(s, \xi^{\prime}, u^{\prime}\right)\right| \leq C\left(1+|u|+\left|u^{\prime}\right|\right)\left(\left|\xi-\xi^{\prime}\right|+\left|u-u^{\prime}\right|\right)$. 
2) P-almost all trajectories of $\bar{H}(s, \xi, u)$ are continuously differentiable in $u$, and

$$
\left(D_{u} \bar{H}\right)(s, \xi, u)=E\left[\left(D_{u} f\right)\left(\xi, \bar{X}_{s}^{1}, u, \bar{v}\left(\xi, \bar{X}_{s}^{1}, u\right)\right)+b_{0}\left(x_{0}, \bar{X}_{s}^{1}, z_{0}\right) z_{0} \mid \mathcal{F}_{T}^{W^{0}}\right],
$$

for all $\xi:=\left(x_{0}, y, z_{0}\right)$, where, with our convention for $\xi,\left(\xi, \bar{X}_{s}^{1}\right):=\left(x_{0}, \bar{X}_{s}^{1}, y, z_{0}, 0\right)$.

3) We have, P-a.s., for all $(s, \xi)$ and all $u, u^{\prime} \in U$,

$$
\left(\left(D_{u} \bar{H}\right)(s, \xi, u)-\left(D_{u} \bar{H}\right)\left(s, \xi, u^{\prime}\right), u-u^{\prime}\right) \leq-\left(\frac{\lambda^{2}-\mu^{2}}{\lambda}\right)\left|u-u^{\prime}\right|^{2} .
$$

For the proof the reader is referred to Appendix 4.

A consequence of the preceding lemma is the strict concavity of the function $\bar{H}(s, \xi,):. U \rightarrow \mathbb{R}$, uniform in $\left(s, \xi=\left(x_{0}, y, z_{0}\right)\right) \in[0, T] \times \mathbb{R}^{3}$ and uniform over $\Omega$. This implies that the random field $\bar{u}$ : $\Omega \times[0, T] \times \mathbb{R}^{3} \rightarrow U, \mathbb{F}^{W^{0}}$-progressively measurable for frozen $\xi=\left(x_{0}, y, z_{0}\right)$, is uniquely defined by the relation

$$
\bar{H}(s, \xi, \bar{u}(s, \xi))=\sup _{u \in U} \bar{H}(s, \xi, u),(s, \xi) \in[0, T] \times \mathbb{R}^{3} .
$$

Lemma 4.3. Under our standard assumptions $\bar{u}: \Omega \times[0, T] \times \mathbb{R}^{3} \rightarrow U$ has the following properties:

$$
\begin{array}{ll}
|\bar{u}(s, \xi)| & \leq C \\
\left|\bar{u}(s, \xi)-\bar{u}\left(s, \xi^{\prime}\right)\right| \leq C\left|\xi-\xi^{\prime}\right|, & \leq
\end{array}
$$

for some constant $C \in \mathbb{R}$ not depending on $s \in[0, T], \xi, \xi^{\prime} \in \mathbb{R}^{3}$.

For the proof the reader is referred to Appendix 4.

Let us now introduce the Hamiltonian of our limit BSDE: We put

$$
\bar{H}(s, \xi)=\bar{H}(s, \xi, \bar{u}(s, \xi)), \xi=\left(x_{0}, y, z_{0}\right) \in \mathbb{R}^{3} .
$$

Then we get immediately from the Lemmas 4.2 and 4.3 that, for some constant $C$ independent of $s \in[0, T]$, $\xi, \xi^{\prime} \in \mathbb{R}^{3}$,

$$
\begin{array}{ll}
|\bar{H}(s, \xi)| & \leq C \\
\left|\bar{H}(s, \xi)-\bar{H}\left(s, \xi^{\prime}\right)\right| & \leq C\left|\xi-\xi^{\prime}\right|
\end{array}
$$

Consequently, as $\bar{H}(., \xi)$ is $\mathbb{F}^{W^{0}}$-progressively measurable, we have the following

Lemma 4.4. There exists a unique solution $\left(\bar{Y}, \bar{Z}^{0}\right) \in \mathcal{S}_{\mathbb{F}^{0}}^{2}(t, T) \times L_{\mathbb{F}^{0}}^{2}(t, T)$ of the BSDE

$$
d \bar{Y}_{s}=-\bar{H}\left(s, X_{s}^{0}, \bar{Y}_{s}, \bar{Z}_{s}^{0}\right) d s+\bar{Z}_{s}^{0} d W_{s}^{0}, s \in[t, T), \quad \bar{Y}_{T}=E\left[\Phi\left(X_{T}^{0}, \bar{X}_{T}^{1}\right) \mid \mathcal{F}_{T}^{W^{0}}\right] .
$$

\subsection{The convergence to the limit game}

The main result in this subsection concerns the convergence of the pay-off/cost functional of the game with $N+1$ participants under saddle point controls to the solution of BSDE (4.12). More precisely, we have

Theorem 4.1. Let $\left(\bar{Y}, \bar{Z}^{0}\right) \in \mathcal{S}_{\mathbb{F}^{0}{ }^{0}}^{2}(t, T) \times L_{\mathbb{F}^{0}}^{2}(t, T)$ be the unique solution of the BSDE 4.12) and $\left(\bar{Y}^{N}, \bar{Z}^{(N)}=\left(\bar{Z}^{0, N}, \ldots, \bar{Z}^{N, N}\right)\right) \in \mathcal{S}_{\mathbb{F}^{N}}^{2}(t, T) \times L_{\mathbb{F}^{N}}^{2}(t, T)^{N+1}$ that of the BSDE (3.17) introduced in Lemma 3.4. Then, under the assumption that $\varepsilon_{N}=O\left(N^{-3 / 4}\right)$, as $N \rightarrow+\infty$, there exists a constant $C \in \mathbb{R}, N_{0} \geq 1$ and, for all $m \geq 1$, a constant $C_{m} \in \mathbb{R}$, such that for all $N \geq N_{0}$,

i) $\left|\bar{Y}_{s}^{N}-\overline{Y_{s}}\right| \leq C\left(\frac{1}{N}+\frac{1}{N} \sum_{l=1}^{N}\left|x_{l}-\bar{x}\right|^{2}\right)^{\frac{1}{2}}, s \in[t, T]$;

ii) $E\left[\left(\int_{t}^{T}\left|\bar{Z}_{s}^{0, N}-\bar{Z}_{s}^{0}\right|^{2} d s+\sum_{l=1}^{N} \int_{t}^{T}\left|\bar{Z}_{s}^{l, N}\right|^{2} d s\right)^{m} \mid \mathcal{F}_{t}^{W^{0}}\right] \leq C_{m}\left(\frac{1}{N}+\frac{1}{N} \sum_{l=1}^{N}\left|x_{l}-\bar{x}\right|^{2}\right)^{m}$. 
Taking into account that we have supposed that $\frac{1}{N} \sum_{l=1}^{N}\left|x_{l}-\bar{x}\right|^{2} \rightarrow 0$, as $N \rightarrow+\infty$, an obvious consequence of the theorem is the following

Corollary 4.1. Under our standard assumptions on the coefficients as well as the condition that $\varepsilon_{N}=$ $O\left(N^{-3 / 4}\right)$, as $N \rightarrow+\infty$, we have for all $m \geq 1$, in $L^{m}$,

$$
\left|\bar{Y}_{s}^{N}-\overline{Y_{s}}\right|^{2}+\int_{t}^{T}\left|\bar{Z}_{s}^{0, N}-\bar{Z}_{s}^{0}\right|^{2} d s+\sum_{l=1}^{N} \int_{t}^{T}\left|\bar{Z}_{s}^{l, N}\right|^{2} d s \longrightarrow 0 .
$$

The proof of the theorem is prepared by two auxiliary lemmas. The first lemma analyzes the limit behavior of the couple of saddle point controls

$$
\bar{u}_{s}^{N}=\bar{u}^{N}\left(X_{s}^{(N)}, \bar{Y}_{s}^{N}, \bar{Z}_{s}^{(N)}\right), \quad \bar{v}_{s}^{(N)}=\left(\bar{v}_{s}^{(\ell, N)}=\bar{v}_{\ell}^{N}\left(X_{s}^{(N)}, \bar{Y}_{s}^{N}, \bar{Z}_{s}^{(N)}\right)\right)_{1 \leq \ell \leq N},
$$

as the number $N$ of collectively playing minor agents tends to infinity. Putting

$$
\bar{u}_{s}:=\bar{u}\left(s, X_{s}^{0}, \bar{Y}_{s}, \bar{Z}_{s}^{0}\right), \quad \bar{v}_{s}^{j}=\bar{v}\left(X_{s}^{0}, \bar{X}_{s}^{j}, \bar{Y}_{s}, \bar{Z}_{s}^{0}, \bar{u}_{s}\right), s \in[t, T]
$$

(recall the definition of $\bar{u}($.$) by (4.8) and that of \bar{v}($.$) by (4.1)), we can establish the following result:$

Lemma 4.5. Under our standard assumption of Lipschitz continuity on the coefficients we have

$$
\begin{aligned}
& \left|\bar{u}_{s}^{N}-\bar{u}_{s}\right|+\left|\bar{v}_{s}^{(j, N)}-\bar{v}_{s}^{j}\right| \\
\leq & C\left(\left|\bar{Y}_{s}^{N}-\bar{Y}_{s}\right|+\left|\bar{Z}_{s}^{0, N}-\bar{Z}_{s}^{0}\right|+\left(1+\varepsilon_{N} \cdot N\right) \frac{1}{N} \sum_{\ell=1}^{N}\left|\bar{Z}_{s}^{\ell, N}\right|\right)+R_{s}^{N},
\end{aligned}
$$

$s \in[t, T], 1 \leq j \leq N, N \geq 1$, where, for all $m \geq 1$, the remainder $R_{s}^{N}$ satisfies the following estimate for some constant $C_{m}$ only depending on $m$ :

$$
E\left[\left|R_{s}^{N}\right|^{2 m} \mid \mathcal{F}_{T}^{W^{0}}\right] \leq C_{m}\left(\frac{1}{N}+\frac{1}{N} \sum_{\ell=1}^{N}\left|x_{\ell}-\bar{x}\right|^{2}\right)^{m}, s \in[t, T], N \geq 1 .
$$

The proof of this lemma is presented in Appendix 5.

The above lemma allows to characterize the limit behavior of the Hamiltonian

$$
\bar{H}_{N}\left(\xi^{(N)}\right):=H_{N}\left(\xi^{(N)}, \bar{u}^{N}\left(\xi^{(N)}\right), \bar{v}^{N}\left(\xi^{(N)}\right)\right)
$$

along the saddle points $\left(\bar{u}^{N}\left(\xi^{(N)}\right), \bar{v}^{N}\left(\xi^{(N)}\right)\right)$, as $N$ tends $+\infty$.

Lemma 4.6. Under the standard assumptions on the coefficients the following estimate with some suitable constant $C$ holds true:

$$
\begin{aligned}
& \left|\bar{H}_{N}\left(X_{s}^{(N)}, \bar{Y}_{s}^{N}, \bar{Z}_{s}^{(N)}\right)-\bar{H}\left(s, X_{s}^{0}, \bar{Y}_{s}, \bar{Z}_{s}^{0}\right)\right| \\
\leq & C\left(\left|\bar{Y}_{s}^{N}-\bar{Y}_{s}\right|+\left|\bar{Z}_{s}^{0, N}-\bar{Z}_{s}^{0}\right|\right)+C\left(\frac{1}{N}+\varepsilon_{N}+\varepsilon_{N}^{2} N\right) \sum_{\ell=1}^{N}\left|\bar{Z}_{s}^{\ell, N}\right|+R_{s}^{N} .
\end{aligned}
$$

As in Lemma 4.5, the remainder $R_{s}^{N}$ satisfies the following estimate:

$$
E\left[\left|R_{s}^{N}\right|^{2 m} \mid \mathcal{F}_{T}^{W^{0}}\right] \leq C_{m}\left(\frac{1}{N}+\frac{1}{N} \sum_{\ell=1}^{N}\left|x_{\ell}-\bar{x}\right|^{2}\right)^{m}, s \in[t, T], N \geq 1, m \geq 1 .
$$

The proof of this lemma is given in Appendix 5 .

With the both preceding lemmas we are now able to prove Theorem 4.1 
Proof. (of Theorem 4.1). Recall that $\left(\bar{Y}^{N}, \bar{Z}^{(N)}\right)$ is introduced in Lemma 3.4 as the unique solution of the BSDE (3.17) with Hamiltonian $\bar{H}_{N}$, while the couple of processes $\left(\bar{Y}, \bar{Z}^{0}\right)$ is the unique solution of BSDE (4.12) with Hamiltonian $\bar{H}$. By applying Itô formula to $\left|\bar{Y}_{s}^{N}-\bar{Y}_{s}\right|^{2}$, we get from BSDE standard estimates

$$
\begin{aligned}
& \left|\bar{Y}_{s}^{N}-\bar{Y}_{s}\right|^{2}+E\left[\int_{s}^{T}\left|\bar{Z}_{r}^{0, N}-\bar{Z}_{r}^{0}\right|^{2} r+\sum_{\ell=1}^{N} \int_{s}^{T}\left|\bar{Z}_{r}^{\ell, N}\right|^{2} d r \mid \mathcal{F}_{s}^{W^{0}}\right] \\
\leq & C E\left[\left|\frac{1}{N} \sum_{\ell=1}^{N} \Phi\left(X_{T}^{0}, X_{T}^{\ell}\right)-E\left[\Phi\left(X_{T}^{0}, \bar{X}_{T}^{\ell}\right) \mid \mathcal{F}_{T}^{W^{0}}\right]\right|^{2} \mid \mathcal{F}_{s}^{W^{0}}\right] \\
& +C E\left[\int_{s}^{T}\left|\bar{Y}_{r}^{N}-\bar{Y}_{r}\right|\left|\bar{H}_{N}\left(X_{r}^{(N)}, \bar{Y}_{r}^{N}, \bar{Z}_{r}^{(N)}\right)-\bar{H}\left(r, X_{r}^{0}, \bar{Y}_{r}, \bar{Z}_{r}^{0}\right)\right| d r \mid \mathcal{F}_{s}^{W^{0}}\right] .
\end{aligned}
$$

From Lemma 2.1 we know that

$$
E\left[\left|\frac{1}{N} \sum_{\ell=1}^{N} \Phi\left(X_{T}^{0}, X_{T}^{\ell}\right)-E\left[\Phi\left(X_{T}^{0}, \bar{X}_{T}^{\ell}\right) \mid \mathcal{F}_{T}^{W^{0}}\right]\right|^{2} \mid \mathcal{F}_{s}^{W^{0}}\right] \leq C\left(\frac{1}{N}+\frac{1}{N} \sum_{\ell=1}^{N}\left|x_{\ell}-\bar{x}\right|^{2}\right) .
$$

Consequently, Lemma 4.6 yields that, for $\rho>0$ small (we will specify $\rho$ later) and a constant $C_{\rho}$ depending on $\rho$,

$$
\begin{aligned}
& \left|\bar{Y}_{s}^{N}-\bar{Y}_{s}\right|^{2}+E\left[\int_{s}^{T}\left|\bar{Z}_{r}^{0, N}-\bar{Z}_{r}^{0}\right|^{2} d r+\sum_{\ell=1}^{N} \int_{s}^{T}\left|\bar{Z}_{r}^{\ell, N}\right|^{2} d r \mid \mathcal{F}_{s}^{W^{0}}\right] \\
\leq & C_{\rho}\left(\frac{1}{N}+\frac{1}{N} \sum_{\ell=1}^{N}\left|x_{\ell}-\bar{x}\right|^{2}\right)+C_{\rho} \int_{s}^{T} E\left[\left|\bar{Y}_{r}^{N}-\bar{Y}_{r}\right|^{2} \mid \mathcal{F}_{s}^{W^{0}}\right] d r+\frac{1}{2} E\left[\int_{s}^{T}\left|\bar{Z}_{r}^{0, N}-\bar{Z}_{r}^{0}\right|^{2} d r \mid \mathcal{F}_{s}^{W^{0}}\right] \\
& +C_{\rho} \int_{s}^{T} E\left[\left|R_{r}^{N}\right|^{2} \mid \mathcal{F}_{s}^{W^{0}}\right] d r+\rho\left(\frac{1}{N}+\varepsilon_{N}+\varepsilon_{N}^{2} N\right)^{2} E\left[\int_{s}^{T}\left(\sum_{\ell=1}^{N}\left|\bar{Z}_{r}^{\ell, N}\right|\right)^{2} d r \mid \mathcal{F}_{s}^{W^{0}}\right],
\end{aligned}
$$

where the estimate of the remainder $R_{r}^{N}, r \in[t, T]$, is given by Lemma 4.6. Obviously,

$$
\left(\frac{1}{N}+\varepsilon_{N}+\varepsilon_{N}^{2} N\right)^{2}\left(\sum_{\ell=1}^{N}\left|\bar{Z}_{r}^{\ell, N}\right|\right)^{2} \leq C\left(\frac{1}{N}+N \varepsilon_{N}^{2}+N^{3} \varepsilon_{N}^{4}\right) \sum_{\ell=1}^{N}\left|\bar{Z}_{r}^{\ell, N}\right|^{2},
$$

and it is here, where we have to use our assumption that $\varepsilon_{N}=O\left(N^{-3 / 4}\right)$, as $N \rightarrow \infty$. Indeed, this assumption allows to get

$$
\rho\left(\frac{1}{N}+\varepsilon_{N}+\varepsilon_{N}^{2} N\right)^{2}\left(\sum_{\ell=1}^{N}\left|\bar{Z}_{s}^{\ell, N}\right|\right)^{2} \leq \frac{1}{2} \sum_{\ell=1}^{N}\left|\bar{Z}_{s}^{\ell, N}\right|^{2}, \quad N \geq 1, s \in[t, T],
$$

for a sufficiently small chosen $\rho>0$. Hence, with such a choice of $\rho$ we obtain

$$
\begin{aligned}
& \left|\bar{Y}_{s}^{N}-\bar{Y}_{s}\right|^{2}+E\left[\int_{s}^{T}\left|\bar{Z}_{r}^{0, N}-\bar{Z}_{r}^{0}\right|^{2} d r+\sum_{\ell=1}^{N} \int_{s}^{T}\left|\bar{Z}_{r}^{\ell, N}\right|^{2} d r \mid \mathcal{F}_{s}^{W^{0}}\right] \\
\leq & C\left(\frac{1}{N}+\frac{1}{N} \sum_{\ell=1}^{N}\left|x_{\ell}-\bar{x}\right|^{2}\right)+C E\left[\int_{s}^{T}\left|\bar{Y}_{r}^{N}-\bar{Y}_{r}\right|^{2} d r \mid \mathcal{F}_{s}^{W^{0}}\right], \quad s \in[t, T], \quad N \geq 1 .
\end{aligned}
$$

Consequently, from Gronwall's inequality we have for all $N \geq 1, P$-a.s.,

$$
\left|\bar{Y}_{s}^{N}-\bar{Y}_{s}\right|^{2}+E\left[\int_{s}^{T}\left|\bar{Z}_{r}^{0, N}-\bar{Z}_{r}^{0}\right|^{2} d r+\sum_{\ell=1}^{N} \int_{s}^{T}\left|\bar{Z}_{r}^{\ell, N}\right|^{2} d r \mid \mathcal{F}_{s}^{W^{0}}\right] \leq C\left(\frac{1}{N}+\frac{1}{N} \sum_{\ell=1}^{N}\left|x_{\ell}-\bar{x}\right|^{2}\right), s \in[t, T],
$$

i.e., estimate (4.13)-i) is proved. It still remains to show ii). For this end, we consider the difference between the BSDEs solved by $\left(\bar{Y}^{N}, \bar{Z}^{(N)}\right)$ and by $\left(\bar{Y}, \bar{Z}^{0}\right)$, and we apply to this difference the Burkholder-DavisGundy inequality. Thus, using Lemma 2.1 and Lemma 4.6. we get, for every $m \geq 1$ and some constant $C_{m}$ depending on $m$,

$$
\begin{aligned}
& E\left[\left(\int_{s}^{s+\delta}\left|\bar{Z}_{r}^{0, N}-\bar{Z}_{r}^{0}\right|^{2} d r+\sum_{\ell=1}^{N} \int_{s}^{s+\delta}\left|\bar{Z}_{r}^{\ell, N}\right|^{2} d r\right)^{m} \mid \mathcal{F}_{t}^{W^{0}}\right] \\
\leq & C_{m} E\left[\sup _{r \in[s, s+\delta]}\left|\int_{s}^{r}\left(\bar{Z}_{v}^{0, N}-\bar{Z}_{v}^{0}\right) d W_{v}^{0}+\sum_{\ell=1}^{N} \int_{s}^{r} \bar{Z}_{v}^{\ell, N} d W_{v}^{\ell}\right|^{2 m} \mid \mathcal{F}_{t}^{W^{0}}\right] \\
\leq & C_{m} E\left[\sup _{r \in[s, s+\delta]}\left|\bar{Y}_{r}^{N}-\bar{Y}_{r}\right|^{2 m}+\left(\int_{s}^{s+\delta}\left|\bar{H}_{N}\left(X_{r}^{(N)}, \bar{Y}_{r}^{N}, \bar{Z}_{r}^{(N)}\right)-\bar{H}\left(r, X_{r}^{0}, \bar{Y}_{r}, \bar{Z}_{r}^{0}\right)\right| d r\right)^{2 m} \mid \mathcal{F}_{t}^{W^{0}}\right] \\
\leq & C_{m}\left(\frac{1}{N}+\frac{1}{N} \sum_{\ell=1}^{N}\left|x_{\ell}-\bar{x}\right|^{2}\right)^{m}+C_{m} E\left[\left(\int_{s}^{s+\delta}\left(\left|\bar{Z}_{r}^{0, N}-\bar{Z}_{r}^{0}\right|+\left(\frac{1}{N}+\varepsilon_{N}+\varepsilon_{N}^{2} N\right) \sum_{\ell=1}^{N}\left|\bar{Z}_{r}^{\ell, N}\right|\right) d r\right)^{2 m} \mid \mathcal{F}_{t}^{W^{0}}\right] \\
\leq & C_{m}\left(\frac{1}{N}+\frac{1}{N} \sum_{\ell=1}^{N}\left|x_{\ell}-\bar{x}\right|^{2}\right)^{m}+C_{m} \delta^{m} E\left[\left(\int_{s}^{s+\delta}\left|\bar{Z}_{r}^{0, N}-\bar{Z}_{r}^{0}\right|^{2} d r+\sum_{\ell=1}^{N} \int_{s}^{s+\delta}\left|\bar{Z}_{r}^{\ell, N}\right|^{2} d r\right)^{m} \mid \mathcal{F}_{t}^{W^{0}}\right],
\end{aligned}
$$

for all $t \leq s<s+\delta \leq T$ and all $N \geq N_{0}$, for some $N_{0} \geq 1$ large enough (recall that $\varepsilon_{N}=O\left(N^{-3 / 4}\right)$ ). Now inequality (4.13)-ii) follows easily. 
After having proved Theorem 4.1, we can combine it with Lemma 4.5 in order to improve its statement concerning the convergence of the saddle point controls of the game with $N$ collectively acting minor agents, when $N$ tends to $+\infty$. Then we obtain easily the following result:

Theorem 4.2. Under our standard assumptions as well as the condition that $\varepsilon_{N}=O\left(N^{-3 / 4}\right)$, as $N \rightarrow+\infty$, we have that for all $m \geq 1$ there is a constant $C_{m} \in \mathbb{R}$, such that for all $N \geq N_{0}$,

i) $\quad E\left[\left(\int_{t}^{T}\left(\left|\bar{u}_{s}^{N}-\bar{u}_{s}\right|+\left|\bar{v}_{s}^{(j, N)}-\bar{v}_{s}^{j}\right|\right)^{2} d s\right)^{m} \mid \mathcal{F}_{t}^{W^{0}}\right] \leq C_{m}\left(\frac{1}{N}+\frac{1}{N} \sum_{\ell=1}^{N}\left|x_{\ell}-\bar{x}\right|^{2}\right)^{m}, 1 \leq j \leq N$;

ii) $\quad E\left[\left(\int_{t}^{T}\left|\frac{1}{N} \sum_{\ell=1}^{N} \psi\left(\bar{v}_{s}^{\ell, N}\right)-E\left[\psi\left(\bar{v}_{s}\right) \mid \mathcal{F}_{T}^{W^{0}}\right]\right|^{2} d s\right)^{m} \mid \mathcal{F}_{t}^{W^{0}}\right]$ $\leq C_{m}\left(\frac{1}{N}+\frac{1}{N} \sum_{\ell=1}^{N}\left|x_{\ell}-\bar{x}\right|^{2}\right)^{m}, 1 \leq j \leq N$, for all bounded Lipschitz functions $\psi$,

where $\bar{v}_{s}:=\bar{v}\left(X_{s}^{0}, \bar{X}_{s}^{1}, \bar{Y}_{s}, \bar{Z}_{s}^{0}, \bar{u}_{s}\right), s \in[t, T]$ (recall that $\bar{v}_{s}^{j}=\bar{v}\left(X_{s}^{0}, \bar{X}_{s}^{j}, \bar{Y}_{s}, \bar{Z}_{s}^{0}, \bar{u}_{s}\right)$ ). As, due to our assumption, $\frac{1}{N} \sum_{\ell=1}^{N}\left|x_{\ell}-\bar{x}\right|^{2} \rightarrow 0$, this means in particular, that the left-hand sides of the above estimates converge to zero, as $N$ tends to $+\infty$.

Remark 4.1. Theorem 4.2 describes the limit behavior of the saddle point controls of the game with $N$ collectively acting minor agents. While statement i) says that the saddle point control of the major player $\bar{u}^{N}$ and, for all $j \geq 1$, that of the $j$-th minor agent $\bar{v}^{j, N}$ converge, for all $m \geq 1$, in $L^{m}\left(\Omega, L^{2}([t, T])\right)$ to the processes $\bar{u}$ and $\bar{v}^{j}$, respectively, statement ii) says that, if we identify in the limit the collectively acting minor agents with a limit player, whose dynamics is $\bar{X}_{s}^{1}=\bar{x}+W_{s}^{1}-W_{t}^{1}, s \in[t, T]$, we get the associated control process $\bar{v}_{s}=\bar{v}\left(X_{s}^{0}, \bar{X}_{s}^{1}, \bar{Y}_{s}, \bar{Z}_{s}^{0}, \bar{u}_{s}\right)$ as a weak limit. More precisely, with the convention $E\left[\delta_{\bar{v}_{s}} \mid \mathcal{F}_{T}^{W^{0}}\right](\psi)=E\left[\delta_{\bar{v}_{s}}(\psi) \mid \mathcal{F}_{T}^{W^{0}}\right]=E\left[\psi\left(\bar{v}_{s}\right) \mid \mathcal{F}_{T}^{W^{0}}\right]$, for $\psi \in C_{b}(\mathbb{R})$,

$$
\frac{1}{N} \sum_{\ell=1}^{N} \delta_{\bar{v}_{s}^{\ell, N}} \rightarrow E\left[\delta_{\bar{v}_{s}} \mid \mathcal{F}_{T}^{W^{0}}\right] \text { weakly, in } L^{m}\left(\Omega, L^{2}([t, T])\right)
$$

Proof. Statement i) is a direct consequence from Lemma 4.5 combined with Theorem 4.1, while statement ii) of the theorem follows easily from i) and Lemma 2.1 .

Recall the definition of $\bar{u}$ and also that

$$
\bar{v}_{s}=\bar{v}\left(X_{s}^{0}, \bar{X}_{s}^{1}, \bar{Y}_{s}, \bar{Z}_{s}^{0}, \bar{u}_{s}\right), s \in[t, T] .
$$

From Lemma 4.1 and the boundedness of the process $\bar{u}$ (Lemma 4.3) it follows that of the process $\bar{v}$. Consequently, from (4.23) we have, for all $m \geq 1$ and all $N \geq N_{0}$,

$$
E\left[\left(\int_{t}^{T}\left|\frac{1}{N} \sum_{\ell=1}^{N} \bar{v}_{s}^{\ell, N}-E\left[\bar{v}_{s} \mid \mathcal{F}_{T}^{W^{0}}\right]\right|^{2} d s\right)^{m} \mid \mathcal{F}_{t}^{W^{0}}\right] \leq C_{m}\left(\frac{1}{N}+\frac{1}{N} \sum_{\ell=1}^{N}\left|x_{\ell}-\bar{x}\right|^{2}\right)^{m} .
$$

Our objective is to characterize the couple $(\bar{u}, \bar{v})$ obtained in Theorem 4.2 as saddle point control for a limit 2-person zero-sum stochastic differential game.

In order to define this 2-person zero-sum game, we introduce the function

$$
F\left(x_{0}, x_{1}, y, z_{0}, u, v\right):=f\left(x_{0}, x_{1}, y, z_{0}, 0, u, v\right)+b_{0}\left(x_{0}, x_{1}, z_{0}\right) z_{0} u,
$$

$\left(x_{0}, x_{1}, y, z_{0}, u, v\right) \in \mathbb{R}^{4} \times U \times V$. We consider as space of admissible controls for Player 1 the set $\overline{\mathcal{U}}=$ $L_{\mathbb{F} W^{0}}^{\infty}(t, T ; U)$ and for Player 2 the set $\overline{\mathcal{V}}=L_{\mathbb{F}^{1}}^{\infty}(t, T ; V)\left(=L_{\mathbb{F} W^{0}, W^{1}}^{\infty}(t, T ; V)\right.$ (recall that $\mathbb{F}^{N}$ is the filtration generated by the Brownian motions $W^{0}, W^{1}, \ldots, W^{N}$ and augmented by all $P$-null sets). Given a couple of admissible controls $(u, v) \in \overline{\mathcal{U}} \times \overline{\mathcal{V}}$, we consider the BSDE

$$
\begin{aligned}
d \bar{Y}_{s}^{u, v} & =-E\left[F\left(X_{s}^{0}, \bar{X}_{s}^{1}, \bar{Y}_{s}^{u, v}, \bar{Z}_{s}^{u, v}, u_{s}, v_{s}\right) \mid \mathcal{F}_{T}^{W^{0}}\right] d s+\bar{Z}_{s}^{u, v} d W_{s}^{0}, s \in[t, T] \\
\bar{Y}_{T}^{u, v} & =E\left[\Phi\left(X_{T}^{0}, \bar{X}_{T}^{1}\right) \mid \mathcal{F}_{T}^{W^{0}}\right]
\end{aligned}
$$


governed by

$$
\begin{array}{ll}
X_{s}^{0}=x_{0}+W_{s}^{0}-W_{t}^{0} & \text {-the dynamics of Player } 1 \text { (the major player) } \\
\bar{X}_{s}^{1}=\bar{x}+W_{s}^{1}-W_{t}^{1} & \text {-the dynamics of Player } 2 \text { (the collectively acting minor agents } \\
& \text { who, in the averaging limit, amalgamate to a single player). }
\end{array}
$$

From our standard assumptions on the functions $f$ and $b_{0}$ we have

$$
\begin{aligned}
\left|F\left(x_{0}, x_{1}, y, z_{0}, u, v\right)\right| & \leq C\left(1+|u|^{2}+|v|^{2}\right), \\
\left|F\left(x_{0}, x_{1}, y, z_{0}, u, v\right)-F\left(x_{0}, x_{1}, y^{\prime}, z_{0}^{\prime}, u, v\right)\right| & \leq C(1+|u|)\left(\left|y-y^{\prime}\right|+\left|z_{0}-z_{0}^{\prime}\right|\right),
\end{aligned}
$$

for all $\left(x_{0}, x_{1}, u, v\right), y, y^{\prime}$ and $z_{0}, z_{0}^{\prime}$. Consequently, from standard BSDE arguments we have

Lemma 4.7. Under our standard assumptions, for any $(u, v) \in \overline{\mathcal{U}} \times \overline{\mathcal{V}}$, BSDE (4.27) has a unique solution $\left(\bar{Y}^{u, v}, \bar{Z}^{u, v}\right) \in \mathcal{S}_{\mathbb{F}^{0}}^{2}(t, T) \times L_{\mathbb{F}^{0}}^{2}(t, T)$. Moreover, for the controls $(\bar{u}, \bar{v}) \in \overline{\mathcal{U}} \times \overline{\mathcal{V}}$ defined respectively in 4.15) and (4.24), the couple $\left(\bar{Y}^{\bar{u}, \bar{v}}, \bar{Z}^{\bar{u}, \bar{v}}\right)$ coincides with the unique solution $\left(\bar{Y}, \bar{Z}^{0}\right)$ of BSDE (4.12).

Proof. The fact that BSDE (4.27) has a unique solution $\left(\bar{Y}^{u, v}, \bar{Z}^{u, v}\right) \in \mathcal{S}_{\mathbb{F}^{0}}^{2}(t, T) \times L_{\mathbb{F}^{0}}^{2}(t, T)$ is an easy consequence of the above properties of the function $F$.

Indeed, thanks to them, given $(u, v) \in \overline{\mathcal{U}} \times \overline{\mathcal{V}}$, the coefficient $E\left[F\left(X_{s}^{0}, \bar{X}_{s}^{1}, y, z_{0}, u_{s}, v_{s}\right) \mid \mathcal{F}_{T}^{W^{0}}\right]$ is, for all $\left(y, z_{0}\right) \in \mathbb{R}^{2}, \mathbb{F}^{W^{0}}$-progressively measurable, Lipschitz in $\left(y, z_{0}\right)$ and bounded, uniformly in $(s, \omega) \in[0, T] \times \Omega$. The second part of the statement, the assertion that $\left(\bar{Y}^{\bar{u}, \bar{v}}, \bar{Z}^{\bar{u}, \bar{v}}\right)=\left(\bar{Y}, \bar{Z}^{0}\right)$, follows the fact that the driving coefficients of the both corresponding BSDEs coincide. Indeed, taking into account the definition of the controls $\bar{u}$ and $\bar{v}$, we have

$$
\begin{aligned}
& E\left[F\left(X_{s}^{0}, \bar{X}_{s}^{1}, \bar{Y}_{s}, \bar{Z}_{s}^{0}, \bar{u}_{s}, \bar{v}_{s}\right) \mid \mathcal{F}_{T}^{W_{0}}\right]=E\left[f_{\bar{v}}\left(X_{s}^{0}, \bar{X}_{s}^{1}, \bar{Y}_{s}, \bar{Z}_{s}^{0}, \bar{u}_{s}\right)+b_{0}\left(X_{s}^{0}, \bar{X}_{s}^{1}, \bar{Z}_{s}^{0}\right) \bar{Z}_{s}^{0} \cdot \bar{u}_{s} \mid \mathcal{F}_{T}^{W_{0}}\right] \\
= & \bar{H}\left(s, X_{s}^{0}, \bar{Y}_{s}, \bar{Z}_{s}^{0}, \bar{u}_{s}\right)=\bar{H}\left(s, X_{s}^{0}, \bar{Y}_{s}, \bar{Z}_{s}^{0}\right) \quad(\text { from (4.10) }), \quad s \in[t, T]
\end{aligned}
$$

(recall that due to our convention $f_{\bar{v}}\left(x_{0}, x_{1}, y, z_{0}, u\right)=f_{\bar{v}}\left(x_{0}, x_{1}, y, z_{0}, 0, u\right)$ ).

The stated result follows now from the uniqueness of the solution for BSDE (4.12).

With the help of BSDE (4.27) let us now introduce the pay-off/cost functional for our 2-person zerosum limit game:

$$
J(u, v):=\bar{Y}_{t}^{u, v}, \quad(u, v) \in \overline{\mathcal{U}} \times \overline{\mathcal{V}} .
$$

Player 1 (the major player) wants to maximize his payoff $J(u, v)$ through the controls $u \in \overline{\mathcal{U}}$, while Player 2the amalgamated collectively acting minor agents - wants to minimize the loss $J(u, v)$ by using the controls $v \in \overline{\mathcal{V}}$.

For the such defined game we have the following characterization:

Theorem 4.3. The limit $(\bar{u}, \bar{v}) \in \overline{\mathcal{U}} \times \overline{\mathcal{V}}$ in the sense of Theorem 4.2 of the couples of saddle point controls $\left(\bar{u}^{N}, \bar{v}^{(N)}\right)$ of the game with $N$ minor agents is a saddle point control for the limit stochastic differential game defined above:

$$
\bar{Y}_{s}^{u, \bar{v}} \leq \bar{Y}_{s}^{\bar{u}, \bar{v}} \leq \bar{Y}_{s}^{\bar{u}, v}, s \in[t, T], P \text {-a.s. }, \quad(u, v) \in \overline{\mathcal{U}} \times \overline{\mathcal{V}},
$$

i.e., in particular, it holds

$$
J(u, \bar{v}) \leq J(\bar{u}, \bar{v}) \leq J(\bar{u}, v), \quad(u, v) \in \overline{\mathcal{U}} \times \overline{\mathcal{V}} .
$$

Proof. Step 1: $\bar{Y}_{s}^{\bar{u}, \bar{v}} \leq \bar{Y}_{s}^{\bar{u}, v}, s \in[t, T], P$-a.s., $v \in \overline{\mathcal{V}}$.

Indeed, given any $v \in \overline{\mathcal{V}}$, we have

$$
\begin{aligned}
F\left(X_{s}^{0}, \bar{X}_{s}^{1}, \bar{Y}_{s}, \bar{Z}_{s}^{0}, \bar{u}_{s}, \bar{v}_{s}\right) & =f_{\bar{v}}\left(X_{s}^{0}, \bar{X}_{s}^{1}, \bar{Y}_{s}, \bar{Z}_{s}^{0}, 0, \bar{u}_{s}\right)+b_{0}\left(X_{s}^{0}, \bar{X}_{s}^{1}, \bar{Z}_{s}^{0}\right) \bar{Z}_{s}^{0} \bar{u}_{s} \\
& \leq f\left(X_{s}^{0}, \bar{X}_{s}^{1}, \bar{Y}_{s}, \bar{Z}_{s}^{0}, 0, \bar{u}_{s}, v_{s}\right)+b_{0}\left(X_{s}^{0}, \bar{X}_{s}^{1}, \bar{Z}_{s}^{0}\right) \bar{Z}_{s}^{0} \bar{u}_{s} \\
& =F\left(X_{s}^{0}, \bar{X}_{s}^{1}, \bar{Y}_{s}, \bar{Z}_{s}^{0}, \bar{u}_{s}, v_{s}\right), \quad s \in[t, T],
\end{aligned}
$$

and, thus,

$$
E\left[F\left(X_{s}^{0}, \bar{X}_{s}^{1}, \bar{Y}_{s}, \bar{Z}_{s}^{0}, \bar{u}_{s}, \bar{v}_{s}\right) \mid \mathcal{F}_{T}^{W^{0}}\right] \leq E\left[F\left(X_{s}^{0}, \bar{X}_{s}^{1}, \bar{Y}_{s}, \bar{Z}_{s}^{0}, \bar{u}_{s}, v_{s}\right) \mid \mathcal{F}_{T}^{W^{0}}\right], s \in[t, T] .
$$


This allows to conclude with the help of the comparison theorem for BSDEs.

Step 2: $\bar{Y}_{s}^{u, \bar{v}} \leq \bar{Y}_{s}^{\bar{u}, \bar{v}}, s \in[t, T], P$-a.s., $u \in \overline{\mathcal{U}}$.

$\overline{\text { Let } u} \in \overline{\mathcal{U}}$. Then, using the definition $\left(\bar{u}_{s}\right)_{0 \leq s \leq T}$ and $\left(\bar{v}_{s}\right)_{0 \leq s \leq T}$ in (4.15) and (4.24), respectively, we have

$$
\begin{aligned}
F\left(X_{s}^{0}, \bar{X}_{s}^{1}, \bar{Y}_{s}, \bar{Z}_{s}^{0}, u_{s}, \bar{v}_{s}\right) & =F\left(X_{s}^{0}, \bar{X}_{s}^{1}, \bar{Y}_{s}, \bar{Z}_{s}^{0}, u_{s}, \bar{v}\left(X_{s}^{0}, \bar{X}_{s}^{1}, \bar{Y}_{s}, \bar{Z}_{s}^{0}, \bar{u}\left(s, X_{s}^{0}, \bar{Y}_{s}, \bar{Z}_{s}^{0}\right)\right)\right) \\
& =F_{\bar{v}}\left(s, X_{s}^{0}, \bar{X}_{s}^{1}, \bar{Y}_{s}, \bar{Z}_{s}^{0}, u_{s}\right),
\end{aligned}
$$

with

$$
F_{\bar{v}}\left(s, x_{0}, x_{1}, y, z_{0}, u\right):=F\left(x_{0}, x_{1}, y, z_{0}, u, \bar{v}\left(x_{0}, x_{1}, y, z_{0}, \bar{u}\left(s, x_{0}, y, z_{0}\right)\right) .\right.
$$

From the properties of $f, b_{0}$ and those of $\bar{u}, \bar{v}$ we obtain

(1) $\left|F_{\bar{v}}(s, \xi, u)-F_{\bar{v}}\left(s, \xi^{\prime}, u\right)\right| \leq C(1+|u|)\left|\xi-\xi^{\prime}\right|, \quad \xi=\left(x_{0}, x_{1}, y, z\right), \xi^{\prime}=\left(x_{0}^{\prime}, x_{1}^{\prime}, y^{\prime}, z^{\prime}\right)$,

(2) $\left|F_{\bar{v}}(s, \xi, u)\right| \leq C\left(1+|u|^{2}\right)$.

Putting

$$
R_{s}^{N}\left(x_{0}, y, z_{0}, u\right):=\left|E\left[F_{\bar{v}}\left(s, x_{0}, \bar{X}_{s}^{1}, y, z_{0}, u\right) \mid \mathcal{F}_{T}^{W^{0}}\right]-\frac{1}{N} \sum_{\ell=1}^{N} F_{\bar{v}}\left(s, x_{0}, X_{s}^{\ell}, y, z_{0}, u\right)\right|,
$$

we have from Lemma 2.1

$$
E\left[\left|R_{s}^{N}\left(x_{0}, y, z_{0}, u\right)\right|^{2} \mid \mathcal{F}_{T}^{W^{0}}\right] \leq C_{K}\left(\frac{1}{N}+\frac{1}{N} \sum_{\ell=1}^{N}\left|x_{\ell}-\bar{x}\right|^{2}\right),
$$

for all $N \geq 1, s \in[t, T], x_{0}, y, z_{0} \in \mathbb{R}$ and $u \in K$, where $K \subset U$ is an arbitrary compact subset of $U$. Consequently, for all $u \in \overline{\mathcal{U}}$,

$$
E\left[\left|R_{s}^{N}\left(X_{s}^{0}, \bar{Y}_{s}, \bar{Z}_{s}^{0}, u_{s}\right)\right|^{2} \mid \mathcal{F}_{T}^{W^{0}}\right] \leq C_{u}\left(\frac{1}{N}+\frac{1}{N}\left|x_{l}-\bar{x}\right|^{2}\right),
$$

and with the notation

$$
R_{s}^{N, u}:=R_{s}^{N}\left(X_{s}^{0}, \bar{Y}_{s}, \bar{Z}_{s}^{0}, u_{s}\right),
$$

it follows from (4.30) and (4.32) that

$$
\begin{aligned}
& E\left[F\left(X_{s}^{0}, \bar{X}_{s}^{1}, \bar{Y}_{s}, \bar{Z}_{s}^{0}, u_{s}, \bar{v}_{s}\right) \mid \mathcal{F}_{T}^{W^{0}}\right] \leq \frac{1}{N} \sum_{\ell=1}^{N} F_{\bar{v}}\left(s, X_{s}^{0}, X_{s}^{\ell}, \bar{Y}_{s}, \bar{Z}_{s}^{0}, u_{s}\right)+R_{s}^{N, u} \\
= & \frac{1}{N} \sum_{\ell=1}^{N} F\left(X_{s}^{0}, X_{s}^{\ell}, \bar{Y}_{s}, \bar{Z}_{s}^{0}, u_{s}, \bar{v}\left(X_{s}^{0}, X_{s}^{\ell}, \bar{Y}_{s}, \bar{Z}_{s}^{0}, \bar{u}\left(s, X_{s}^{0}, \bar{Y}_{s}, \bar{Z}_{s}^{0}\right)\right)\right)+R_{s}^{N, u} \\
= & \frac{1}{N} \sum_{\ell=1}^{N} F\left(X_{s}^{0}, X_{s}^{\ell}, \bar{Y}_{s}, \bar{Z}_{s}^{0}, u_{s}, \bar{v}\left(X_{s}^{0}, X_{s}^{\ell}, \bar{Y}_{s}, \bar{Z}_{s}^{0}, \bar{u}_{s}\right)\right)+R_{s}^{N, u} \\
\leq & \frac{1}{N} \sum_{\ell=1}^{N} F\left(X_{s}^{0}, X_{s}^{\ell}, \bar{Y}_{s}, \bar{Z}_{s}^{0}, u_{s}, \bar{v}\left(X_{s}^{0}, X_{s}^{\ell}, \bar{Y}_{s}, \bar{Z}_{s}^{0}, \bar{u}_{s}^{N, 0}\right)\right)+R_{s}^{N, u}+C_{u}\left|\bar{u}_{s}-\bar{u}_{s}^{N, 0}\right|,
\end{aligned}
$$

where

$$
\bar{u}_{s}^{N, 0}:=\bar{u}^{N}\left(X_{s}^{(N)}, \bar{Y}_{s}^{N},\left(\bar{Z}_{s}^{0, N}, 0, \cdots, 0\right)\right) .
$$

The latter estimate was obtained thanks to the Lipschitz continuity of $F\left(x_{0}, x_{1}, y, z_{0}, u, v\right)$ in $v$ and that of $\bar{v}\left(x_{0}, x_{1}, y, z_{0}, u\right)$ in $u$.

Thus, using the uniform Lipschitz continuity of $f$ and $b_{0}\left(x_{0}, x_{1}, z_{0}\right) z_{0}$ in $\left(y, z_{0}\right)$, we get

$$
\begin{aligned}
& E\left[F\left(X_{s}^{0}, \bar{X}_{s}^{1}, \bar{Y}_{s}, \bar{Z}_{s}^{0}, u_{s}, \bar{v}_{s}\right) \mid \mathcal{F}_{T}^{W^{0}}\right] \\
\leq \quad & \frac{1}{N} \sum_{\ell=1}^{N} F\left(X_{s}^{0}, X_{s}^{\ell}, \bar{Y}_{s}, \bar{Z}_{s}^{0}, u_{s}, \bar{v}\left(X_{s}^{0}, X_{s}^{\ell}, \bar{Y}_{s}, \bar{Z}_{s}^{0}, \bar{u}_{s}^{N, 0}\right)\right)+R_{s}^{N, u}+C_{u}\left|\bar{u}_{s}-\bar{u}_{s}^{N, 0}\right| \\
\leq \quad & \frac{1}{N} \sum_{\ell=1}^{N} F\left(X_{s}^{0}, X_{s}^{\ell}, \bar{Y}_{s}^{N}, \bar{Z}_{s}^{0, N}, u_{s}, \bar{v}\left(X_{s}^{0}, X_{s}^{\ell}, \bar{Y}_{s}^{N}, \bar{Z}_{s}^{0, N}, \bar{u}_{s}^{N, 0}\right)\right)+R_{s}^{N, u} \\
& +C\left(\left|\bar{Y}_{s}^{N}-\bar{Y}_{s}\right|+\left|\bar{Z}_{s}^{0, N}-\bar{Z}_{s}^{0}\right|\right)+C_{u}\left|\bar{u}_{s}-\bar{u}_{s}^{N, 0}\right| .
\end{aligned}
$$

Observe that from (3.12) we have

$$
\begin{aligned}
\bar{v}_{\ell, s}^{N, 0} & \left.:=\bar{v}_{\ell}^{N}\left(X_{s}^{(N)}, \bar{Y}_{s}^{N},\left(\bar{Z}_{s}^{0, N}, 0, \cdots, 0\right)\right)\right) \\
& =\widetilde{v}_{N}\left(X_{s}^{0}, X_{s}^{\ell}, \bar{Y}_{s}^{N},\left(\bar{Z}_{s}^{0, N}, 0, \cdots, 0\right), \bar{u}_{s}^{N, 0}\right) \\
& =\bar{v}\left(X_{s}^{0}, X_{s}^{\ell}, \bar{Y}_{s}^{N}, \bar{Z}_{s}^{0, N}, \bar{u}_{s}^{N, 0}\right) .
\end{aligned}
$$


The latter equality follows from the fact that, if $z_{\ell}=0,1 \leq \ell \leq N$, then the minimizer $\bar{v}(\xi, u)$ of $f(\xi, u,$. (see (4.1)) and the minimizer $\widetilde{v}_{N}(\xi, u)$ in (3.11) satisfy the relation

$$
\widetilde{v}_{N}\left(x_{0}, x_{\ell}, y,\left(z_{0}, 0, \ldots, 0\right), u\right)=\bar{v}\left(x_{0}, x_{\ell}, y, z_{0}, u\right) .
$$

Therefore, using definition (4.26) of $F$, we can write

$$
\begin{aligned}
& E\left[F\left(X_{s}^{0}, \bar{X}_{s}^{1}, \bar{Y}_{s}, \bar{Z}_{s}^{0}, u_{s}, \bar{v}_{s}\right) \mid \mathcal{F}_{T}^{W^{0}}\right] \\
\leq & \left(\frac{1}{N} \sum_{\ell=1}^{N} f\left(X_{s}^{0}, X_{s}^{\ell}, \bar{Y}_{s}^{N}, \bar{Z}_{s}^{0, N}, 0, u_{s}, \bar{v}_{\ell, s}^{N, 0}\right)+\frac{1}{N} \sum_{\ell=1}^{N} b_{0}\left(X_{s}^{0}, X_{s}^{\ell}, \bar{Z}_{s}^{0, N}\right) \bar{Z}_{s}^{0, N} \cdot u_{s}\right) \\
& +R_{s}^{N, u}+C\left(\left|\bar{Y}_{s}^{N}-\bar{Y}_{s}\right|+\left|\bar{Z}_{s}^{0, N}-\bar{Z}_{s}^{0}\right|\right)+C_{u}\left|\bar{u}_{s}-\bar{u}_{s}^{N, 0}\right| \\
= & H_{N}\left(X_{s}^{(N)}, \bar{Y}_{s}^{N},\left(\bar{Z}_{s}^{0, N}, 0, \cdots, 0\right), u_{s}, \bar{v}_{s}^{N, 0}\right)+R_{s}^{N, u}+C\left(\left|\bar{Y}_{s}^{N}-\bar{Y}_{s}\right|+\left|\bar{Z}_{s}^{0, N}-\bar{Z}_{s}^{0}\right|\right)+C_{u}\left|\bar{u}_{s}-\bar{u}_{s}^{N, 0}\right| \\
\leq & H_{N}\left(X_{s}^{(N)}, \bar{Y}_{s}^{N},\left(\bar{Z}_{s}^{0, N}, 0, \cdots, 0\right), \bar{u}_{s}^{0, N}, \bar{v}_{s}^{N, 0}\right)+R_{s}^{N, u}+C\left(\left|\bar{Y}_{s}^{N}-\bar{Y}_{s}\right|+\left|\bar{Z}_{s}^{0, N}-\bar{Z}_{s}^{0}\right|\right)+C_{u}\left|\bar{u}_{s}-\bar{u}_{s}^{N, 0}\right| .
\end{aligned}
$$

Here we have used that $\left(\bar{u}_{s}^{N}, \bar{v}_{s}^{N, 0}=\left(\bar{v}_{1, s}^{N, 0}, \ldots, \bar{v}_{N, s}^{N, 0}\right)\right)$ is the saddle point control for the Hamiltonian $H_{N}\left(X_{s}^{(N)}, \bar{Y}_{s}^{N},\left(\bar{Z}_{s}^{0, N}, 0, \ldots, 0\right), \cdot, \cdot\right)$. On the other hand, since the controls $\bar{u}^{N, 0}$ and $\bar{u}$ are bounded by some constant not depending on $N$ (see Lemma 3.2 and take into account that here $z_{\ell}=0,1 \leq \ell \leq N$ ), we obtain

$$
\begin{aligned}
& H_{N}\left(X_{s}^{(N)}, \bar{Y}_{s}^{N},\left(\bar{Z}_{s}^{0, N}, 0, \cdots, 0\right), \bar{u}_{s}^{N, 0}, \bar{v}_{s}^{N, 0}\right) \\
= & \frac{1}{N} \sum_{\ell=1}^{N} f_{\bar{v}}\left(X_{s}^{0}, X_{s}^{\ell}, \bar{Y}_{s}^{N}, \bar{Z}_{s}^{0, N}, 0, \bar{u}_{s}^{N, 0}\right)+\frac{1}{N} \sum_{\ell=1}^{N} b_{0}\left(X_{s}^{0}, X_{s}^{\ell}, \bar{Z}_{s}^{0, N}\right) \bar{Z}_{s}^{0, N} \cdot \bar{u}_{s}^{N, 0} \\
\leq & \frac{1}{N} \sum_{\ell=1}^{N} f_{\bar{v}}\left(X_{s}^{0}, X_{s}^{\ell}, \bar{Y}_{s}, \bar{Z}_{s}^{0}, 0, \bar{u}_{s}\right)+\frac{1}{N} \sum_{\ell=1}^{N} b_{0}\left(X_{s}^{0}, X_{s}^{\ell}, \bar{Z}_{s}^{0}\right) \bar{Z}_{s}^{0} \cdot \bar{u}_{s} \\
& +C\left(\left|\bar{Y}_{s}^{N}-\bar{Y}_{s}\right|+\left|\bar{Z}_{s}^{0, N}-\bar{Z}_{s}^{0}\right|\right)+C\left|\bar{u}_{s}^{N, 0}-\bar{u}_{s}\right| \\
\leq & E\left[f_{\bar{v}}\left(X_{s}^{0}, \bar{X}_{s}^{1}, \bar{Y}_{s}, \bar{Z}_{s}^{0}, 0, \bar{u}_{s}\right)+b_{0}\left(X_{s}^{0}, \bar{X}_{s}^{1}, \bar{Z}_{s}^{0}\right) \bar{Z}_{s}^{0} \cdot \bar{u}_{s} \mid \mathcal{F}_{T}^{W^{0}}\right]+C\left(\left|\bar{Y}_{s}^{N}-\bar{Y}_{s}\right|+\left|\bar{Z}_{s}^{0, N}-\bar{Z}_{s}^{0}\right|\right) \\
& +C\left|\bar{u}_{s}^{N, 0}-\bar{u}_{s}\right|+R_{s}^{N} \\
= & E\left[F\left(X_{s}^{0}, \bar{X}_{s}^{1}, \bar{Y}_{s}, \bar{Z}_{s}^{0}, \bar{u}_{s}, \bar{v}_{s}\right) \mid \mathcal{F}_{T}^{W^{0}}\right]+R_{s}^{N}+C\left(\left|\bar{Y}_{s}^{N}-\bar{Y}_{s}\right|+\left|\bar{Z}_{s}^{0, N}-\bar{Z}_{s}^{0}\right|\right)+C\left|\bar{u}_{s}^{N, 0}-\bar{u}_{s}\right|,
\end{aligned}
$$

where $E\left[\left(R_{s}^{N}\right)^{2} \mid \mathcal{F}_{T}^{W^{0}}\right] \leq C\left(\frac{1}{N}+\frac{1}{N} \sum_{\ell=1}^{N}\left|x_{\ell}-\bar{x}\right|^{2}\right)$, and we have used Lemma 2.1 for the latter estimate. Hence, combining the above estimates of Step 2, we get

$$
\begin{aligned}
& E\left[F\left(X_{s}^{0}, \bar{X}_{s}^{1}, \bar{Y}_{s}, \bar{Z}_{s}^{0}, u_{s}, \bar{v}_{s}\right) \mid \mathcal{F}_{T}^{W^{0}}\right] \\
\leq & E\left[F\left(X_{s}^{0}, \bar{X}_{s}^{1}, \bar{Y}_{s}, \bar{Z}_{s}^{0}, \bar{u}_{s}, \bar{v}_{s}\right) \mid \mathcal{F}_{T}^{W^{0}}\right]+C\left(\left|\bar{Y}_{s}^{N}-\bar{Y}_{s}\right|+\left|\bar{Z}_{s}^{0, N}-\bar{Z}_{s}^{0}\right|\right)+C\left|\bar{u}_{s}^{N, 0}-\bar{u}_{s}\right|+R_{s}^{N},
\end{aligned}
$$

where

$$
E\left[\left(R_{s}^{N}\right)^{2} \mid \mathcal{F}_{T}^{W^{0}}\right] \leq C\left(\frac{1}{N}+\frac{1}{N} \sum_{\ell=1}^{N}\left|x_{\ell}-\bar{x}\right|^{2}\right), N \geq 1, s \in[t, T] .
$$

But, since due to Lemma 4.5 (recall that $\bar{u}_{s}^{N, 0}=\bar{u}^{N}\left(X_{s}^{(N)}, \bar{Y}_{s}^{N},\left(\bar{Z}_{s}^{0, N}, 0 \ldots, 0\right)\right)$ ),

we conclude

$$
\left|\bar{u}_{s}^{N, 0}-\bar{u}_{s}\right| \leq C\left(\left|\bar{Y}_{s}^{N}-\bar{Y}_{s}\right|+\left|\bar{Z}_{s}^{0, N}-\bar{Z}_{s}^{0}\right|\right)+R_{s}^{N} .
$$

$$
\begin{aligned}
& E\left[F\left(X_{s}^{0}, \bar{X}_{s}^{1}, \bar{Y}_{s}, \bar{Z}_{s}^{0}, u_{s}, \bar{v}_{s}\right) \mid \mathcal{F}_{T}^{W^{0}}\right] \\
\leq \quad & E\left[F\left(X_{s}^{0}, \bar{X}_{s}^{1}, \bar{Y}_{s}, \bar{Z}_{s}^{0}, \bar{u}_{s}, \bar{v}_{s}\right) \mid \mathcal{F}_{T}^{W^{0}}\right]+C\left(\left|\bar{Y}_{s}^{N}-\bar{Y}_{s}\right|+\left|\bar{Z}_{s}^{0, N}-\bar{Z}_{s}^{0}\right|\right)+R_{s}^{N},
\end{aligned}
$$

and, thanks to Theorem 4.1, as $N \rightarrow \infty$,

$$
E\left[F\left(X_{s}^{0}, \bar{X}_{s}^{1}, \bar{Y}_{s}, \bar{Z}_{s}^{0}, u_{s}, \bar{v}_{s}\right) \mid \mathcal{F}_{T}^{W^{0}}\right] \leq E\left[F\left(X_{s}^{0}, \bar{X}_{s}^{1}, \bar{Y}_{s}, \bar{Z}_{s}^{0}, \bar{u}_{s}, \bar{v}_{s}\right) \mid \mathcal{F}_{T}^{W^{0}}\right], d s d P \text {-a.e. }
$$

Consequently, from the comparison theorem for BSDEs we have

$$
\bar{Y}_{s}^{\bar{u}, \bar{v}}=\bar{Y}_{s} \geq \bar{Y}_{s}^{u, \bar{v}}, s \in[t, T], u \in \overline{\mathcal{U}},
$$

and the proof is complete now. 
While we have seen in Theorem 4.3 that $(\bar{u}, \bar{v})$ is a saddle point control of our limit 2-person zero-sum game, it turns out that it is even the unique saddle point control. Indeed, we have the following uniqueness result:

Theorem 4.4. Let $\left(u^{\prime}, v^{\prime}\right),\left(u^{\prime \prime}, v^{\prime \prime}\right) \in \overline{\mathcal{U}} \times \overline{\mathcal{V}}$ be two couples of saddle point controls in the sense that, for all $(u, v) \in \overline{\mathcal{U}} \times \overline{\mathcal{V}}$,

$$
\begin{aligned}
& J\left(u, v^{\prime}\right) \leq J\left(u^{\prime}, v^{\prime}\right) \leq J\left(u^{\prime}, v\right), \\
& J\left(u, v^{\prime \prime}\right) \leq J\left(u^{\prime \prime}, v^{\prime \prime}\right) \leq J\left(u^{\prime \prime}, v\right) .
\end{aligned}
$$

Then $\left(u_{s}^{\prime}, v_{s}^{\prime}\right)=\left(u_{s}^{\prime \prime}, v_{s}^{\prime \prime}\right)$, dsdP-a.e.

Proof. Let $\left(u^{\prime}, v^{\prime}\right),\left(u^{\prime \prime}, v^{\prime \prime}\right) \in \overline{\mathcal{U}} \times \overline{\mathcal{V}}$ be two couples of saddle point controls in the above sense.

Step 1. Let $u \in \mathcal{U}$. Then $J\left(u^{\prime}, v^{\prime}\right) \geq J\left(u, v^{\prime}\right), P$-a.s., and it follows that $\bar{Y}_{s}^{u^{\prime}, v^{\prime}} \geq \bar{Y}_{s}^{u, v^{\prime}}, s \in[t, T]$, and

$$
E\left[F\left(X_{s}^{0}, \bar{X}_{s}^{1}, \bar{Y}_{s}^{u^{\prime}, v^{\prime}}, \bar{Z}_{s}^{u^{\prime}, v^{\prime}}, u_{s}^{\prime}, v_{s}^{\prime}\right) \mid \mathcal{F}_{T}^{W^{0}}\right] \geq E\left[F\left(X_{s}^{0}, \bar{X}_{s}^{1}, \bar{Y}_{s}^{u^{\prime}, v^{\prime}}, \bar{Z}_{s}^{u^{\prime}, v^{\prime}}, u_{s}, v_{s}^{\prime}\right) \mid \mathcal{F}_{T}^{W^{0}}\right] \text {, dsdP-a.e. }
$$

Indeed, putting $\gamma_{s}:=I\left\{E\left[F\left(X_{s}^{0}, \bar{X}_{s}^{1}, \bar{Y}_{s}^{u^{\prime}, v^{\prime}}, \bar{Z}_{s}^{u^{\prime}, v^{\prime}}, u_{s}^{\prime}, v_{s}^{\prime}\right) \mid \mathcal{F}_{T}^{W^{0}}\right] \geq E\left[F\left(X_{s}^{0}, \bar{X}_{s}^{1}, \bar{Y}_{s}^{u^{\prime}, v^{\prime}}, \bar{Z}_{s}^{u^{\prime}, v^{\prime}}, u_{s}, v_{s}^{\prime}\right) \mid \mathcal{F}_{T}^{W^{0}}\right]\right\}$, the process $\tilde{u}_{s}:=u_{s}^{\prime} \gamma_{s}+u_{s}\left(1-\gamma_{s}\right), s \in[t, T]$, defines an admissible control in $\overline{\mathcal{U}}$, and

$$
E\left[F\left(X_{s}^{0}, \bar{X}_{s}^{1}, \bar{Y}_{s}^{u^{\prime}, v^{\prime}}, \bar{Z}_{s}^{u^{\prime}, v^{\prime}}, \tilde{u}_{s}, v_{s}^{\prime}\right) \mid \mathcal{F}_{T}^{W^{0}}\right] \geq E\left[F\left(X_{s}^{0}, \bar{X}_{s}^{1}, \bar{Y}_{s}^{u^{\prime}, v^{\prime}}, \bar{Z}_{s}^{u^{\prime}, v^{\prime}}, u_{s}^{\prime}, v_{s}^{\prime}\right) \mid \mathcal{F}_{T}^{W^{0}}\right], s \in[t, T] .
$$

Consequently, the comparison theorem yields $\bar{Y}_{s}^{\tilde{u}, v^{\prime}} \geq \bar{Y}_{s}^{u^{\prime}, v^{\prime}}$, P-a.s., $s \in[t, T]$. But, since on the other hand, $J\left(\tilde{u}, v^{\prime}\right) \leq J\left(u^{\prime}, v^{\prime}\right)$, it follows from the converse comparison theorem that $\bar{Y}_{s}^{\tilde{u}, v^{\prime}}=\bar{Y}_{s}^{u^{\prime}, v^{\prime}}$, P-a.s., $s \in[t, T]$, and

$$
E\left[F\left(X_{s}^{0}, \bar{X}_{s}^{1}, \bar{Y}_{s}^{u^{\prime}, v^{\prime}}, \bar{Z}_{s}^{u^{\prime}, v^{\prime}}, \tilde{u}_{s}, v_{s}^{\prime}\right) \mid \mathcal{F}_{T}^{W^{0}}\right]=E\left[F\left(X_{s}^{0}, \bar{X}_{s}^{1}, \bar{Y}_{s}^{u^{\prime}, v^{\prime}}, \bar{Z}_{s}^{u^{\prime}, v^{\prime}}, u_{s}^{\prime}, v_{s}^{\prime}\right) \mid \mathcal{F}_{T}^{W^{0}}\right], \text { dsdP-a.e. }
$$

But this implies

$$
E\left[F\left(X_{s}^{0}, \bar{X}_{s}^{1}, \bar{Y}_{s}^{u^{\prime}, v^{\prime}}, \bar{Z}_{s}^{u^{\prime}, v^{\prime}}, u_{s}, v_{s}^{\prime}\right) \mid \mathcal{F}_{T}^{W^{0}}\right] \leq E\left[F\left(X_{s}^{0}, \bar{X}_{s}^{1}, \bar{Y}_{s}^{u^{\prime}, v^{\prime}}, \bar{Z}_{s}^{u^{\prime}, v^{\prime}}, u_{s}^{\prime}, v_{s}^{\prime}\right) \mid \mathcal{F}_{T}^{W^{0}}\right], \text { dsdP-a.e. }
$$

from where we see that $\bar{Y}_{s}^{u^{\prime}, v^{\prime}} \geq \bar{Y}_{s}^{u, v^{\prime}}, s \in[t, T]$, and $u_{s}^{\prime}$ is a maximum point of $u \rightarrow E\left[F\left(X_{s}^{0}, \bar{X}_{s}^{1}, \bar{Y}_{s}^{u^{\prime}, v^{\prime}}\right.\right.$, $\left.\left.\bar{Z}_{s}^{u^{\prime}, v^{\prime}}, u, v_{s}^{\prime}\right) \mid \mathcal{F}_{T}^{W^{0}}\right]$, dsdP-a.e. On the other hand, let us also observe that, from the definition of $F$ and the assumptions on $f$,

$$
\begin{aligned}
& \left(D_{u} E\left[F\left(X_{s}^{0}, \bar{X}_{s}^{1}, \bar{Y}_{s}^{u^{\prime}, v^{\prime}}, \bar{Z}_{s}^{u^{\prime}, v^{\prime}}, u^{1}, v_{s}^{\prime}\right) \mid \mathcal{F}_{T}^{W^{0}}\right]-D_{u} E\left[F\left(X_{s}^{0}, \bar{X}_{s}^{1}, \bar{Y}_{s}^{u^{\prime}, v^{\prime}}, \bar{Z}_{s}^{u^{\prime}, v^{\prime}}, u^{2}, v_{s}^{\prime}\right) \mid \mathcal{F}_{T}^{W^{0}}\right], u^{1}-u^{2}\right) \\
& \leq-\lambda\left|u^{1}-u^{2}\right|^{2}, u^{1}, u^{2} \in U .
\end{aligned}
$$

Consequently, $u_{s}^{\prime}$ is the unique maximum point of $u \rightarrow E\left[F\left(X_{s}^{0}, \bar{X}_{s}^{1}, \bar{Y}_{s}^{u^{\prime}, v^{\prime}}, \bar{Z}_{s}^{u^{\prime}, v^{\prime}}, u, v_{s}^{\prime}\right) \mid \mathcal{F}_{T}^{W^{0}}\right]$.

Step 2. Let $v \in \overline{\mathcal{V}}$. Following the argument in Step 1, but with putting

$$
\gamma_{s}:=I\left\{F\left(X_{s}^{0}, \bar{X}_{s}^{1}, \bar{Y}_{s}^{u^{\prime}, v^{\prime}}, \bar{Z}_{s}^{u^{\prime}, v^{\prime}}, u_{s}^{\prime}, v_{s}^{\prime}\right) \leq F\left(X_{s}^{0}, \bar{X}_{s}^{1}, \bar{Y}_{s}^{u^{\prime}, v^{\prime}}, \bar{Z}_{s}^{u^{\prime}, v^{\prime}}, u_{s}^{\prime}, v_{s}\right)\right\}
$$

and $\tilde{v}_{s}:=v_{s}^{\prime} \gamma_{s}+v_{s}\left(1-\gamma_{s}\right), s \in[t, T]$, we have $\tilde{v} \in \overline{\mathcal{V}}$, and

$$
F\left(X_{s}^{0}, \bar{X}_{s}^{1}, \bar{Y}_{s}^{u^{\prime}, v^{\prime}}, \bar{Z}_{s}^{u^{\prime}, v^{\prime}}, u_{s}^{\prime}, \tilde{v}_{s}\right) \leq F\left(X_{s}^{0}, \bar{X}_{s}^{1}, \bar{Y}_{s}^{u^{\prime}, v^{\prime}}, \bar{Z}_{s}^{u^{\prime}, v^{\prime}}, u_{s}^{\prime}, v_{s}^{\prime}\right), s \in[t, T] .
$$

Consequently, from the comparison theorem it follows that $\bar{Y}_{s}^{u^{\prime}, \tilde{v}} \leq \bar{Y}_{s}^{u^{\prime}, v^{\prime}}$, P-a.s., $s \in[t, T]$, and since on the other hand, $J\left(u^{\prime}, \tilde{v}\right) \geq J\left(u^{\prime}, v^{\prime}\right)$, the converse comparison theorem implies $\bar{Y}_{s}^{u^{\prime}, \tilde{v}}=\bar{Y}_{s}^{u^{\prime}, v^{\prime}}$, P-a.s., $s \in[t, T]$, and

$$
E\left[F\left(X_{s}^{0}, \bar{X}_{s}^{1}, \bar{Y}_{s}^{u^{\prime}, v^{\prime}}, \bar{Z}_{s}^{u^{\prime}, v^{\prime}}, u_{s}^{\prime}, \tilde{v}_{s}\right) \mid \mathcal{F}_{T}^{W^{0}}\right]=E\left[F\left(X_{s}^{0}, \bar{X}_{s}^{1}, \bar{Y}_{s}^{u^{\prime}, v^{\prime}}, \bar{Z}_{s}^{u^{\prime}, v^{\prime}}, u_{s}^{\prime}, v_{s}^{\prime}\right) \mid \mathcal{F}_{T}^{W^{0}}\right] \text {, dsdP-a.e. }
$$


Thus,

$$
F\left(X_{s}^{0}, \bar{X}_{s}^{1}, \bar{Y}_{s}^{u^{\prime}, v^{\prime}}, \bar{Z}_{s}^{u^{\prime}, v^{\prime}}, u_{s}^{\prime}, \tilde{v}_{s}\right)=F\left(X_{s}^{0}, \bar{X}_{s}^{1}, \bar{Y}_{s}^{u^{\prime}, v^{\prime}}, \bar{Z}_{s}^{u^{\prime}, v^{\prime}}, u_{s}^{\prime}, v_{s}^{\prime}\right), \text { dsdP-a.e. }
$$

and

$$
F\left(X_{s}^{0}, \bar{X}_{s}^{1}, \bar{Y}_{s}^{u^{\prime}, v^{\prime}}, \bar{Z}_{s}^{u^{\prime}, v^{\prime}}, u_{s}^{\prime}, v_{s}\right) \geq F\left(X_{s}^{0}, \bar{X}_{s}^{1}, \bar{Y}_{s}^{u^{\prime}, v^{\prime}}, \bar{Z}_{s}^{u^{\prime}, v^{\prime}}, u_{s}^{\prime}, v_{s}^{\prime}\right) \text {, dsdP-a.e. }
$$

which implies that $\bar{Y}_{s}^{u^{\prime}, v^{\prime}} \leq \bar{Y}_{s}^{u^{\prime}, v}, s \in[t, T]$, but also that $v_{s}^{\prime}$ is a minimum point of $v \rightarrow F\left(X_{s}^{0}, \bar{X}_{s}^{1}\right.$, $\left.\bar{Y}_{s}^{u^{\prime}, v^{\prime}}, \bar{Z}_{s}^{u^{\prime}, v^{\prime}}, u_{s}^{\prime}, v\right)$. On the other hand, since

$$
\begin{aligned}
& \left(D_{v} F\left(X_{s}^{0}, \bar{X}_{s}^{1}, \bar{Y}_{s}^{u^{\prime}, v^{\prime}}, \bar{Z}_{s}^{u^{\prime}, v^{\prime}}, u^{\prime}, v^{1}\right)-D_{v} F\left(X_{s}^{0}, \bar{X}_{s}^{1}, \bar{Y}_{s}^{u^{\prime}, v^{\prime}}, \bar{Z}_{s}^{u^{\prime}, v^{\prime}}, u^{\prime}, v^{2}\right), v^{1}-v^{2}\right) \\
& \geq \lambda\left|v^{1}-v^{2}\right|^{2}, v^{1}, v^{2} \in V,
\end{aligned}
$$

it follows that $v_{s}^{\prime}$ is the unique minimum point of $v \rightarrow F\left(X_{s}^{0}, \bar{X}_{s}^{1}, \bar{Y}_{s}^{u^{\prime}, v^{\prime}}, \bar{Z}_{s}^{u^{\prime}, v^{\prime}}, u_{s}^{\prime}, v\right)$.

Step 3. Let $\left(u^{\prime}, v^{\prime}\right),\left(u^{\prime \prime}, v^{\prime \prime}\right) \in \overline{\mathcal{U}} \times \overline{\mathcal{V}}$ be two couples of saddle point controls. Then, combining our results from the Steps 1 and 2 we have

i.e.,

$$
\bar{Y}_{s}^{u^{\prime}, v^{\prime}} \geq \bar{Y}_{s}^{u^{\prime \prime}, v^{\prime}} \geq \bar{Y}_{s}^{u^{\prime \prime}, v^{\prime \prime}} \geq \bar{Y}_{s}^{u^{\prime}, v^{\prime \prime}} \geq \bar{Y}_{s}^{u^{\prime}, v^{\prime}}, s \in[t, T],
$$

$$
\bar{Y}_{s}^{u^{\prime}, v^{\prime}}=\bar{Y}_{s}^{u^{\prime \prime}, v^{\prime}}=\bar{Y}_{s}^{u^{\prime \prime}, v^{\prime \prime}}=\bar{Y}_{s}^{u^{\prime}, v^{\prime \prime}}=\bar{Y}_{s}^{u^{\prime}, v^{\prime}}, s \in[t, T] .
$$

But from $\bar{Y}_{s}^{u^{\prime}, v^{\prime}}=\bar{Y}_{s}^{u^{\prime \prime}, v^{\prime}}, s \in[t, T]$, and the uniqueness of the semimartingale decomposition of this process we deduce that $\bar{Z}_{s}^{u^{\prime}, v^{\prime}}=\bar{Z}_{s}^{u^{\prime \prime}, v^{\prime}}$ dsdP-a.e., and also

$$
E\left[F\left(X_{s}^{0}, \bar{X}_{s}^{1}, \bar{Y}_{s}^{u^{\prime}, v^{\prime}}, \bar{Z}_{s}^{u^{\prime}, v^{\prime}}, u_{s}^{\prime \prime}, v_{s}^{\prime}\right) \mid \mathcal{F}_{T}^{W^{0}}\right]=E\left[F\left(X_{s}^{0}, \bar{X}_{s}^{1}, \bar{Y}_{s}^{u^{\prime}, v^{\prime}}, \bar{Z}_{s}^{u^{\prime}, v^{\prime}}, u_{s}^{\prime}, v_{s}^{\prime}\right) \mid \mathcal{F}_{T}^{W^{0}}\right], \text { dsdP-a.e. }
$$

But this means that also $u_{s}^{\prime \prime}$ is a maximum point of $E\left[F\left(X_{s}^{0}, \bar{X}_{s}^{1}, \bar{Y}_{s}^{u^{\prime}, v^{\prime}}, \bar{Z}_{s}^{u^{\prime}, v^{\prime}}, ., v_{s}^{\prime}\right) \mid \mathcal{F}_{T}^{W^{0}}\right]$, dsdP-a.e., and, thus, due to Step $1, u_{s}^{\prime}=u_{s}^{\prime \prime}$, dsdP-a.e.

Let us now show that also $v_{s}^{\prime}=v_{s}^{\prime \prime}$, dsdP-a.e. As we have seen already above, $\bar{Y}_{s}^{u^{\prime}, v^{\prime}}=\bar{Y}_{s}^{u^{\prime \prime}, v^{\prime \prime}}, s \in$ $[t, T]$, and from the uniqueness of the semimartingale decomposition of this process we get $\bar{Z}_{s}^{u^{\prime}, v^{\prime}}=\bar{Z}_{s}^{u^{\prime \prime}, v^{\prime \prime}}$, dsdP-a.e., and

$$
E\left[F\left(X_{s}^{0}, \bar{X}_{s}^{1}, \bar{Y}_{s}^{u^{\prime}, v^{\prime}}, \bar{Z}_{s}^{u^{\prime}, v^{\prime}}, u_{s}^{\prime \prime}, v_{s}^{\prime \prime}\right) \mid \mathcal{F}_{T}^{W^{0}}\right]=E\left[F\left(X_{s}^{0}, \bar{X}_{s}^{1}, \bar{Y}_{s}^{u^{\prime}, v^{\prime}}, \bar{Z}_{s}^{u^{\prime}, v^{\prime}}, u^{\prime}, v_{s}^{\prime}\right) \mid \mathcal{F}_{T}^{W^{0}}\right], \text { dsdP-a.e. }
$$

Since, on the other hand, for $v=v^{\prime \prime}$ in Step 2,

$$
F\left(X_{s}^{0}, \bar{X}_{s}^{1}, \bar{Y}_{s}^{u^{\prime}, v^{\prime}}, \bar{Z}_{s}^{u^{\prime}, v^{\prime}}, u_{s}^{\prime}, v_{s}^{\prime \prime}\right) \geq F\left(X_{s}^{0}, \bar{X}_{s}^{1}, \bar{Y}_{s}^{u^{\prime}, v^{\prime}}, \bar{Z}_{s}^{u^{\prime}, v^{\prime}}, u_{s}^{\prime}, v_{s}^{\prime}\right), \text { dsdP-a.e. }
$$

it follows that

$$
F\left(X_{s}^{0}, \bar{X}_{s}^{1}, \bar{Y}_{s}^{u^{\prime}, v^{\prime}}, \bar{Z}_{s}^{u^{\prime}, v^{\prime}}, u_{s}^{\prime}, v_{s}^{\prime \prime}\right)=F\left(X_{s}^{0}, \bar{X}_{s}^{1}, \bar{Y}_{s}^{u^{\prime}, v^{\prime}}, \bar{Z}_{s}^{u^{\prime}, v^{\prime}}, u_{s}^{\prime}, v_{s}^{\prime}\right), \text { dsdP-a.e. }
$$

i.e., also $v_{s}^{\prime \prime}$ is a minimum point of $F\left(X_{s}^{0}, \bar{X}_{s}^{1}, \bar{Y}_{s}^{u^{\prime}, v^{\prime}}, \bar{Z}_{s}^{u^{\prime}, v^{\prime}}, u_{s}^{\prime},.\right)$, dsdP-a.e. But due to Step 2, the unique minimum point is $v_{s}^{\prime}$. Consequently, $v_{s}^{\prime}=v_{s}^{\prime \prime}$, dsdP-a.e.

Since we have already shown that $u_{s}^{\prime}=u_{s}^{\prime \prime}$, dsdP-a.e., we conclude that the saddle point controls $\left(u^{\prime}, v^{\prime}\right)$ and $\left(u^{\prime \prime}, v^{\prime \prime}\right)$ coincide.

\section{Appendix}

\subsection{Appendix 1}

Appendix 1 is devoted to the proof of Proposition 2.3 . 
Proof. (of Proposition 2.3). Without loss of the generality, we may suppose that $b_{0}=0$ and $b_{1}=0$. We note that with this convention and with the notation

$$
\sigma_{u}^{1, j, N}:=\frac{1}{N} \sum_{\ell=1}^{N} \sigma_{1}\left(X_{u}^{0, N}, X_{u}^{j, N}, X_{u}^{\ell, N}\right)-E\left[\sigma_{1}\left(\bar{X}_{u}^{0}, \bar{X}_{u}^{j}, \bar{X}_{u}^{j+1}\right) \mid \mathcal{F}_{T}^{W^{0}, W^{j}}\right],
$$

for $t \leq r \leq s \leq T$,

$$
X_{r}^{j, N}-\bar{X}_{r}^{j}=\left(x_{j}-\bar{x}\right)+\int_{t}^{r} \sigma_{u}^{1, j, N} d W_{u}^{j}
$$

from where we deduce

$$
\begin{aligned}
& \frac{1}{N} \sum_{j=1}^{N}\left|X_{r}^{j, N}-\bar{X}_{r}^{j}\right|^{2} \\
\leq & \frac{2}{N} \sum_{j=1}^{N}\left|x_{j}-\bar{x}\right|^{2}+\frac{2}{N} \sum_{j=1}^{N}\left|\int_{t}^{r} \sigma_{u}^{1, j, N} d W_{u}^{j}\right|^{2} \\
= & \frac{2}{N} \sum_{j=1}^{N}\left|x_{j}-\bar{x}\right|^{2}+2\left|M_{r}^{(N)}\right|^{2},
\end{aligned}
$$

where

$$
M_{r}^{(N)}=\sum_{j=1}^{N} e_{j} \int_{t}^{r} \frac{1}{\sqrt{N}} \sigma_{u}^{1, j, N} d W_{u}^{j}, r \in[t, T]
$$

is an $\ell_{2}\left(\mathbb{R}^{d}\right)$-valued $\mathbb{F}$-martingale, and $e_{j}=\left(\delta_{j, k}\right)_{k \geq 1}$ is the element of $\ell_{2}$ with $\delta_{j, j}=1$ and $\delta_{j, k}=0, k \neq j$. Consequently, due to the Burkholder-Davis-Gundy Inequality, for some constant $C_{m} \in \mathbb{R}$ which can vary from line to line but doesn't depend on $N$,

$$
\begin{aligned}
& E\left[\sup _{r \in[t, s]}\left(\frac{1}{N} \sum_{j=1}^{N}\left|X_{r}^{j, N}-\bar{X}_{r}^{j}\right|^{2}\right)^{m}\right] \\
\leq & C_{m}\left(\frac{1}{N} \sum_{j=1}^{N}\left|x_{j}-\bar{x}\right|^{2}\right)^{m}+C_{m} E\left[\sup _{r \in[t, s]}\left|M_{r}^{(N)}\right|^{2 m}\right] \\
\leq & C_{m}\left(\frac{1}{N} \sum_{j=1}^{N}\left|x_{j}-\bar{x}\right|^{2}\right)^{m}+C_{m} E\left[\left\langle M^{(N)}\right\rangle_{s}^{m}\right],
\end{aligned}
$$

where

$$
\left\langle M^{(N)}\right\rangle_{s}=\frac{1}{N} \sum_{j=1}^{N} \int_{t}^{s}\left|\sigma_{u}^{1, j, N}\right|^{2} d u, s \in[t, T],
$$

is the quadratic variation process of the martingale $M^{(N)}=\left(M_{s}^{(N)}\right)_{s \in[t, T]}$. Hence, with the notation

$$
\zeta_{1, j, \ell}(r):=\sigma_{1}\left(\bar{X}_{r}^{0}, \bar{X}_{r}^{j}, \bar{X}_{r}^{\ell}\right)-E\left[\sigma_{1}\left(\bar{X}_{r}^{0}, \bar{X}_{r}^{j}, \bar{X}_{r}^{\ell}\right) \mid \mathcal{F}_{T}^{W^{0}, W^{j}}\right],
$$

we have

$$
\begin{array}{rlr} 
& E\left[\sup _{r \in[t, s]}\left(\frac{1}{N} \sum_{j=1}^{N}\left|X_{r}^{j, N}-\bar{X}_{r}^{j}\right|^{2}\right)^{m}\right] \\
\leq & C_{m}\left(\frac{1}{N} \sum_{j=1}^{N}\left|x_{j}-\bar{x}\right|^{2}\right)^{m}+C_{m} E\left[\left(\frac{1}{N} \sum_{j=1}^{N} \int_{t}^{s}\left|\sigma_{r}^{1, j, N}\right|^{2} d r\right)^{m}\right] \\
\leq \quad & C_{m}\left(\frac{1}{N} \sum_{j=1}^{N}\left|x_{j}-\bar{x}\right|^{2}\right)^{m} \\
& +C_{m} E\left[\left(\int_{t}^{s}\left|X_{r}^{0, N}-\bar{X}_{r}^{0}\right|^{2} d r\right)^{m}\right] \\
& +C_{m} E\left[\left(\frac{1}{N} \sum_{j=1}^{N} \int_{t}^{s}\left|X_{r}^{j, N}-\bar{X}_{r}^{j}\right|^{2} d r+\int_{t}^{s}\left(\frac{1}{N} \sum_{\ell=1}^{N}\left|X_{r}^{\ell, N}-\bar{X}_{r}^{\ell}\right|^{2}\right) d r\right)^{m}\right]\left(=: J_{s}^{2, N}\right) \\
& +C_{m} E\left[\left(\frac{1}{N} \sum_{j=1}^{N} \int_{t}^{s}\left|\frac{1}{N} \sum_{\ell=1, \ell \neq j}^{N} \zeta_{1, j, \ell}(r)\right|^{2} d r\right)^{m}\right] \\
& +\frac{C_{m}}{N^{2 m}} .
\end{array}
$$

The term $J_{s}^{2, N}$ will be handled by Gronwall's Lemma. Let us begin with estimating $J_{s}^{1, N}$. Obviously, putting

$$
\begin{aligned}
& \sigma_{r}^{0, N}:=\frac{1}{N} \sum_{\ell=1}^{N} \sigma_{0}\left(X_{r}^{0, N}, X_{r}^{\ell, N}\right)-E\left[\sigma_{0}\left(\bar{X}_{r}^{0}, \bar{X}_{r}^{1}\right) \mid \mathcal{F}_{T}^{W^{0}}\right], \quad \text { and } \\
& \zeta_{0, \ell}(r):=\sigma_{0}\left(\bar{X}_{r}^{0}, \bar{X}_{r}^{\ell}\right)-E\left[\sigma_{0}\left(\bar{X}_{r}^{0}, \bar{X}_{r}^{\ell}\right) \mid \mathcal{F}_{T}^{W^{0}}\right], \quad r \in[t, T]
\end{aligned}
$$

we obtain,

$$
\begin{aligned}
& E\left[\sup _{r \in[t, s]}\left|X_{r}^{0, N}-\bar{X}_{r}^{0}\right|^{2 m}\right] \leq C_{m} E\left[\left(\int_{t}^{s}\left|\sigma_{r}^{0, N}\right|^{2} d r\right)^{m}\right] \\
\leq \quad & C_{m} E\left[\left(\int_{t}^{s}\left|X_{r}^{0, N}-\bar{X}_{r}^{0}\right|^{2} d r\right)^{m}\right]+C_{m} E\left[\left(\frac{1}{N} \sum_{\ell=1}^{N} \int_{t}^{s}\left|X_{r}^{\ell, N}-\bar{X}_{r}^{\ell}\right|^{2} d r\right)^{m}\right] \\
& +C_{m} E\left[\left(\int_{t}^{s}\left|\frac{1}{N} \sum_{\ell=1}^{N} \zeta_{0, \ell}(r)\right|^{2} d r\right)^{m}\right] \quad\left(=: J_{s}^{4, N}\right) .
\end{aligned}
$$

The following estimates of $J_{s}^{3, N}$ and $J_{s}^{4, N}$ allow to complete the proof by applying Grownwall's Lemma to the system (5.4)-(5.6). 
Lemma 5.1. For some constant $C_{m}$ independent of $N \geq 1$, with the notation $\Lambda_{j, N}:=\{1, \ldots, N\} \backslash\{j\}$, we have

$$
E\left[\left|\sum_{\ell=1}^{N} \zeta_{0, \ell}(r)\right|^{2 m} \mid \mathcal{F}_{T}^{W^{0}}\right] \leq C_{m} N^{m}, E\left[\left|\sum_{\ell \in \Lambda_{j, N}} \zeta_{1, j, \ell}(r)\right|^{2 m} \mid \mathcal{F}_{T}^{W^{0}, W^{j}}\right] \leq C_{m} N^{m}, N \geq 1
$$

Proof. We give the proof only for $E\left[\left|\sum_{\ell \in \Lambda_{j, N}} \zeta_{j, \ell}(r)\right|^{2 m} \mid \mathcal{F}_{T}^{W^{0}, W^{j}}\right]$, that for $E\left[\left|\sum_{\ell=1}^{N} \zeta_{0, \ell}(r)\right|^{2 m} \mid \mathcal{F}_{T}^{W^{0}}\right]$ is analogous. For simplifying the argument we assume that $d=1$. For the multi-dimensional case $d>1$ the random variable $\zeta_{1, j, \ell}(r)$ is matrix-valued and the argument we develop shall be applied component-wise. For our argument we will need the set

$$
\Gamma_{m, N, j}:=\left\{\left(\ell_{1}, \ldots, \ell_{2 m}\right) \in \Lambda_{j, N}^{2 m} \mid \forall i(1 \leq i \leq 2 m), \exists i^{\prime} \in\{1, \ldots, 2 m\} \backslash\{i\}: \ell_{i}=\ell_{i^{\prime}}\right\} .
$$

Remark that the cardinal number of $\Gamma_{m, N, j}$ satisfies the estimate

$$
\operatorname{card}\left(\Gamma_{m, N, j}\right) \leq C_{m} N^{m}, N \geq 1,
$$

for some $C_{m} \in R$ not depending on $N$.

We begin the proof of the lemma by remarking that knowing $\mathcal{F}_{T}^{W^{0}, W^{j}}$ the processes $\bar{X}^{\ell}, \ell \geq 1$ with $\ell \neq j$, are conditionally i.i.d. Consequently, also the random variables $\zeta_{1, j, \ell}(r), \ell \in\{1, \ldots, N\} \backslash\{j\}$, are conditionally i.i.d., knowing $\mathcal{F}_{T}^{W^{0}, W^{j}}$. Moreover, $E\left[\zeta_{1, j, \ell}(r) \mid \mathcal{F}_{T}^{W^{0}, W^{j}}\right]=0$. Thus,

$$
\begin{aligned}
& E\left[\left|\frac{1}{\sqrt{N}} \sum_{\ell \in \Lambda_{j, N}} \zeta_{1, j, \ell}(r)\right|^{2 m} \mid \mathcal{F}_{T}^{W^{0}, W^{j}}\right]=\frac{1}{N^{m}} \sum_{\ell_{1}, \ldots, \ell_{2 m} \in \Lambda_{j, N}} E\left[\prod_{p=1}^{2 m} \zeta_{1, j, \ell_{p}}(r) \mid \mathcal{F}_{T}^{W^{0}, W^{j}}\right] \\
& =\frac{1}{N^{m}} \sum_{\left(\ell_{1}, \ldots, \ell_{2 m}\right) \in \Gamma_{m, N, j}} E\left[\prod_{p=1}^{2 m} \zeta_{1, j, \ell_{p}}(r) \mid \mathcal{F}_{T}^{W^{0}, W^{j}}\right] \\
& \leq \frac{1}{N^{m}} \sum_{\left(\ell_{1}, \ldots, \ell_{2 m}\right) \in \Gamma_{m, N, j}} \prod_{p=1}^{2 m}\left(E\left[\left|\zeta_{1, j, \ell_{p}}(r)\right|^{2 m} \mid \mathcal{F}_{T}^{W^{0}, W^{j}}\right]\right)^{\frac{1}{2 m}} .
\end{aligned}
$$

In virtue of the boundedness of $\sigma_{1}$ and the estimate of the cardinal number of $\Gamma_{m, N, j}$ this yields

$$
E\left[\left|\frac{1}{\sqrt{N}} \sum_{\ell \in \Lambda_{j, N}} \zeta_{1, j, \ell}(r)\right|^{2 m} \mid \mathcal{F}_{T}^{W^{0}, W^{j}}\right] \leq C_{m}
$$

for some $C_{m} \in \mathbb{R}$ independent of $N \geq 1$. The statement of the lemma follows easily from this.

\subsection{Appendix 2}

This appendix is devoted to the proof of Proposition 2.4

Proof. (of Proposition 2.4). Taking the difference between the BSDE for $Y^{N}$ and that for $\bar{Y}$ we obtain

$$
\begin{array}{lrl}
Y_{s}^{N}-\bar{Y}_{s}=\left\{\frac{1}{N} \sum_{\ell=1}^{N} \Phi\left(X_{T}^{0, N}, X_{T}^{\ell, N}\right)-E\left[\Phi\left(\bar{X}_{T}^{0}, \bar{X}_{T}^{1}\right) \mid \mathcal{F}_{T}^{W^{0}}\right]\right\} & \left(:=\eta^{1, N}\right) \\
+\int_{s}^{T}\left(\frac{1}{N} \sum_{\ell=1}^{N}\left(f\left(X_{r}^{0, N}, X_{r}^{\ell, N}, Y_{r}^{N}, Z_{r}^{0, N}, Z_{r}^{\ell, N}\right)-f\left(\bar{X}_{r}^{0}, \bar{X}_{r}^{\ell}, Y_{r}^{N}, Z_{r}^{0, N}, Z_{r}^{\ell, N}\right)\right)\right) d r & \left(:=\int_{s}^{T} \eta_{r}^{2, N} d r\right) \\
+\int_{s}^{T}\left(\frac{1}{N} \sum_{\ell=1}^{N}\left(f\left(\bar{X}_{r}^{0}, \bar{X}_{r}^{\ell}, Y_{r}^{N}, Z_{r}^{0, N}, Z_{r}^{\ell, N}\right)-f\left(\bar{X}_{r}^{0}, \bar{X}_{r}^{\ell}, \bar{Y}_{r}, \bar{Z}_{r}^{0}, 0\right)\right)\right) d r & \left(:=\int_{s}^{T} \eta_{r}^{3, N} d r\right) \\
+\int_{s}^{T}\left(\frac{1}{N} \sum_{\ell=1}^{N} f\left(\bar{X}_{r}^{0}, \bar{X}_{r}^{\ell}, \bar{Y}_{r}, \bar{Z}_{r}^{0}, 0\right)-E\left[f\left(\bar{X}_{r}^{0}, \bar{X}_{r}^{1}, \bar{Y}_{r}, \bar{Z}_{r}^{0}, 0\right) \mid \mathcal{F}_{T}^{W^{0}}\right]\right) d r & \\
\left.-\int_{s}^{T}\left(Z_{r}^{0, N}-\bar{Z}_{r}^{0}\right) d W_{r}^{0}-\sum_{\ell=1}^{N} \int_{s}^{T} Z_{r}^{\ell, N} d W_{r}^{\ell, N} d r\right) & \\
&
\end{array}
$$


From Proposition 2.3 and Lemma 2.1 we know already that

$$
E\left[\left|\eta^{1, N}\right|^{2 m}\right] \leq C_{m}\left(\frac{1}{N}+\frac{1}{N} \sum_{\ell=1}^{N}\left|x_{\ell}-\bar{x}\right|^{2}\right)^{m}, N \geq 1,
$$

for some constant $C_{m}$ independent of $N$. Furthermore, again from Proposition 2.3 we have

$$
\begin{aligned}
& E\left[\int_{t}^{T}\left|\eta_{r}^{2, N}\right|^{2 m} d r\right] \leq C_{m} E\left[\sup _{r \in[t, T]}\left(\left|X_{r}^{0, N}-\bar{X}_{r}^{0}\right|^{2}+\frac{1}{N} \sum_{\ell=1}^{N}\left|X_{r}^{\ell, N}-\bar{X}_{r}^{\ell}\right|^{2}\right)^{m}\right] \\
& \leq C_{m}\left(\frac{1}{N}+\frac{1}{N} \sum_{\ell=1}^{N}\left|x_{\ell}-\bar{x}\right|^{2}\right)^{m} .
\end{aligned}
$$

As concerns the convergence of

$$
\int_{s}^{T} \eta_{r}^{4, N} d r=\int_{s}^{T}\left(\frac{1}{N} \sum_{\ell=1}^{N} f\left(\bar{X}_{r}^{0}, \bar{X}_{r}^{\ell}, \bar{Y}_{r}, \bar{Z}_{r}^{0}, 0\right)-E\left[f\left(\bar{X}_{r}^{0}, \bar{X}_{r}^{1}, \bar{Y}_{r}, \bar{Z}_{r}^{0}, 0\right) \mid \mathcal{F}_{T}^{W^{0}}\right]\right) d r,
$$

the same argument as that for the proof of Lemma 5.1 can be used. Indeed, recalling that the function $f$ is bounded and that the processes $\bar{X}^{0}, \bar{Y}$ and $\bar{Z}^{0}$ are $\mathbb{F}^{W^{0}}$-adapted, we can use the fact that the processes $\bar{X}^{\ell}, \ell \geq 1$, are conditionally i.i.d., knowing $\mathcal{F}_{T}^{W^{0}}$, in order to conclude that

$$
E\left[\int_{t}^{T}\left|\eta_{r}^{4, N}\right|^{2 m} d r\right] \leq \frac{C_{m}}{N^{m}}, \quad N \geq 1
$$

The above estimates allow now to get by a BSDE standard argument that

$$
\begin{aligned}
& E\left[\sup _{s \in[t, T]}\left|Y_{s}^{N}-\bar{Y}_{s}\right|^{2 m}+\left(\int_{t}^{T}\left|Z_{s}^{0, N}-\bar{Z}_{s}^{0}\right|^{2} d s+\sum_{\ell=1}^{N} \int_{t}^{T}\left|Z_{s}^{\ell, N}\right|^{2} d s\right)^{m}\right] \\
& \leq C_{m}\left(\frac{1}{N}+\frac{1}{N} \sum_{\ell=1}^{N}\left|x_{\ell}-\bar{x}\right|^{2}\right)^{m} .
\end{aligned}
$$

The proof is complete.

\subsection{Appendix 3}

Proof. (of Lemma 3.1). For convenience we omit the dependence on $\xi^{(N)}$, we deduce from the fact that $\left(\bar{u}^{N}, \bar{v}^{(N)}\right)$ is the saddle point of $H_{N}$ and the definition of $\left(\tilde{u}^{N}, \tilde{v}^{(N)}\right)$ at one hand

$$
\begin{aligned}
& H_{N}\left(\bar{u}^{N}, \bar{v}^{(N)}\right) \geq H_{N}\left(\tilde{u}^{N}, \bar{v}^{(N)}\right) \geq H_{N}\left(\tilde{u}^{N}, \tilde{v}^{(N)}\left(\tilde{u}^{N}\right)\right) \\
& \geq H_{N}\left(\bar{u}^{N}, \tilde{v}^{(N)}\left(\bar{u}^{N}\right)\right) \geq H_{N}\left(\bar{u}^{N}, \bar{v}^{(N)}\right),
\end{aligned}
$$

i.e., for all $u \in U$,

$$
H_{N}\left(\tilde{u}^{N}, \bar{v}^{(N)}\right)=H_{N}\left(\bar{u}^{N}, \bar{v}^{(N)}\right) \geq H_{N}\left(u, \bar{v}^{(N)}\right) .
$$

Consequently, $\tilde{u}^{N}, \bar{u}^{N} \in U$ are both maximum points of the strict concave function $H_{N}\left(., \bar{v}^{(N)}\right.$ ) (see (3.8)). This implies $\tilde{u}^{N}=\bar{u}^{N}$. On the other hand, using this equality we have

$$
H_{N}\left(\bar{u}^{N}, \tilde{v}^{(N)}\left(\tilde{u}^{N}\right)\right)=H_{N}\left(\tilde{u}^{N}, \tilde{v}^{(N)}\left(\tilde{u}^{N}\right)\right) \leq H_{N}\left(\tilde{u}^{N}, v\right)=H_{N}\left(\bar{u}^{N}, v\right),
$$

i.e., $\tilde{v}^{(N)}\left(\tilde{u}^{N}\right) \in V^{N}$ is a minimum point of the strict convex function $H_{N}\left(\bar{u}^{N}\right.$,.) (see (3.8) again), and it follows that $\tilde{v}^{(N)}\left(\tilde{u}^{N}\right)=\bar{v}^{(N)}$.

We continue with the proof of Lemma 3.2 , 
Proof. (of Lemma 3.2). Let $\xi^{(N)}=\left(x^{(N)}=\left(x_{0}, \ldots, x_{N}\right), y, z^{(N)}=\left(z_{0}, \ldots, z_{N}\right)\right), \xi_{\ell}^{(N)}=\left(x_{0}, x_{\ell}, y, z^{(N)}\right)$ and, given an arbitrary $u \in U, \tilde{v}_{N, \ell}:=\tilde{v}_{N}\left(\xi_{\ell}^{(N)}, u\right), 1 \leq \ell \leq N$. Since the function $v^{(N)} \rightarrow H_{N}\left(\xi^{(N)}, u, v^{(N)}\right)$ achieves its minimum at $\tilde{v}^{(N)}\left(\xi^{(N)}, u\right)$, we have, for all $1 \leq \ell \leq N$,

$$
\begin{aligned}
& 0=N\left(D_{v_{\ell}} H_{N}\right)\left(\xi^{(N)}, u, \tilde{v}^{(N)}\left(\xi^{(N)}, u\right)\right) \\
& =\left(D_{v_{\ell}} f\right)\left(x_{0}, x_{\ell}, y, z_{0}, z_{\ell}, u, \tilde{v}_{N, \ell}\right)+\varepsilon_{N} \sum_{i=1}^{N} b_{1}\left(x_{0}, x_{\ell}, z_{i}\right) z_{i} .
\end{aligned}
$$

Thus,

$$
\begin{aligned}
0= & \left(\left(D_{v_{\ell}} f\right)\left(x_{0}, x_{\ell}, y, z_{0}, z_{\ell}, u, \tilde{v}_{N, \ell}\right)-\left(D_{v_{\ell}} f\right)\left(x_{0}, x_{\ell}, y, z_{0}, z_{\ell}, u, 0\right), \tilde{v}_{N, \ell}\right) \\
& +\left(\left(D_{v_{\ell}} f\right)\left(x_{0}, x_{\ell}, y, z_{0}, z_{\ell}, u, 0\right)+\varepsilon_{N} \sum_{i=1}^{N} b_{1}\left(x_{0}, x_{\ell}, z_{i}\right) z_{i}, \tilde{v}_{N, \ell}\right),
\end{aligned}
$$

and using that

$$
\left|\sum_{i=1}^{N} b_{1}\left(x_{0}, x_{\ell}, z_{i}\right) z_{i}\right| \leq \min \left\{C \sum_{i=1}^{N}\left|z_{i}\right|, C N\right\}, \text { for all }\left(x_{0}, x_{\ell}, z_{i}\right) \in \mathbb{R}^{3}
$$

we deduce

$$
\left|\tilde{v}_{N}\left(\xi_{\ell}^{(N)}, u\right)\right| \leq C+\frac{\mu}{\lambda}|u|+C \varepsilon_{N} \min \left\{\sum_{i=1}^{N}\left|z_{i}\right|, N\right\} .
$$

On the other hand, since $u \rightarrow H_{N}\left(\xi^{(N)}, u, \bar{v}^{(N)}\left(\xi^{(N)}\right)\right)$ achieves a maximum at $\tilde{u}^{N}\left(\xi^{(N)}\right)\left(=\bar{u}^{N}\left(\xi^{(N)}\right)\right)$, with the notation $\xi_{\ell}=\left(x_{0}, x_{\ell}, y, z_{0}, z_{\ell}\right)$ it holds

$$
\begin{aligned}
0= & \frac{1}{N} \sum_{\ell=1}^{N}\left(\left(D_{u} f\right)\left(\xi_{\ell}, \tilde{u}^{N}\left(\xi^{(N)}\right), \bar{v}_{\ell}^{N}\left(\xi^{(N)}\right)\right)-\left(D_{u} f\right)\left(\xi_{\ell}, 0, \bar{v}_{\ell}^{N}\left(\xi^{(N)}\right)\right), \tilde{u}^{N}\left(\xi^{(N)}\right)\right) \\
& +\left(\frac{1}{N} \sum_{\ell=1}^{N}\left(D_{u} f\right)\left(\xi_{\ell}, 0, \bar{v}_{\ell}^{N}\left(\xi^{(N)}\right)\right)+\frac{1}{N} \sum_{\ell=1}^{N} b_{0}\left(x_{0}, x_{\ell}, z_{0}\right) z_{0}, \tilde{u}^{N}\left(\xi^{(N)}\right)\right) \\
\leq & -\lambda\left|\tilde{u}^{N}\left(\xi^{(N)}\right)\right|^{2}+\left(C+\frac{\mu}{N} \sum_{\ell=1}^{N}\left|\bar{v}_{\ell}^{N}\left(\xi^{(N)}\right)\right|\right)\left|\tilde{u}^{N}\left(\xi^{(N)}\right)\right| .
\end{aligned}
$$

Hence,

$$
\begin{aligned}
\left|\tilde{u}^{N}\left(\xi^{(N)}\right)\right| & \leq C+\frac{\mu}{\lambda} \frac{1}{N} \sum_{\ell=1}^{N}\left|\bar{v}_{\ell}^{N}\left(\xi^{(N)}\right)\right| \\
& =C+\frac{\mu}{\lambda} \frac{1}{N} \sum_{\ell=1}^{N}\left|\tilde{v}_{N}\left(\xi_{\ell}^{(N)}, \tilde{u}^{N}\left(\xi^{(N)}\right)\right)\right| \\
& \leq C+\frac{\mu}{\lambda} \frac{1}{N} \sum_{\ell=1}^{N}\left(C+\frac{\mu}{\lambda}\left|\tilde{u}^{N}\left(\xi^{(N)}\right)\right|\right)+\varepsilon_{N} N C \\
& \leq C+C \varepsilon_{N} N+\left(\frac{\mu}{\lambda}\right)^{2}\left|\tilde{u}^{N}\left(\xi^{(N)}\right)\right| .
\end{aligned}
$$

From $\mu<\lambda$, it follows that

$$
\left|\tilde{u}^{N}\left(\xi^{(N)}\right)\right| \leq C\left(1+\varepsilon_{N} N\right),
$$

and since $\bar{v}^{(N)}\left(\xi^{(N)}\right)=\left(\tilde{v}_{N}\left(\xi_{l}^{(N)}, \tilde{u}^{N}\left(\xi^{(N)}\right)\right)\right)_{1 \leq l \leq N}$, we conclude that

$$
\begin{aligned}
& \left|\bar{v}_{\ell}^{N}\left(\xi^{(N)}\right)\right| \leq C\left(1+\varepsilon_{N} N\right), \quad 1 \leq \ell \leq N, \text { i.e., } \\
& \left|\bar{v}^{(N)}\left(\xi^{(N)}\right)\right| \leq \sqrt{N} C\left(1+\varepsilon_{N} N\right) .
\end{aligned}
$$

On the other hand, from (5.20),

$$
0 \leq-\lambda\left|\tilde{u}^{N}\left(\xi^{(N)}\right)\right|^{2}+\left(C+\frac{\mu}{N} \sum_{\ell=1}^{N}\left|\tilde{v}_{N}\left(\xi_{\ell}^{(N)}, \tilde{u}^{N}\left(\xi^{(N)}\right)\right)\right|\right)\left|\tilde{u}^{N}\left(\xi^{(N)}\right)\right|,
$$

we see that

$$
\left|\tilde{u}^{N}\left(\xi^{(N)}\right)\right| \leq C+\frac{\mu}{\lambda}\left(C+\frac{\mu}{\lambda}\left|\tilde{u}^{N}\left(\xi^{(N)}\right)\right|+C \varepsilon_{N} \sum_{i=1}^{N}\left|z_{i}\right|\right),
$$

from where, since $0<\mu<\lambda$, we get that

$$
\left|\tilde{u}^{N}\left(\xi^{(N)}\right)\right| \leq C\left(1+\varepsilon_{N} \sum_{i=1}^{N}\left|z_{i}\right|\right)
$$


Finally, putting (5.19), (5.22) and (5.24) together, we complete the proof of the growth conditions (i) and (ii) for the saddle point control.

It remains to prove the Lipschitz properties (iii) and (iv). For this end, we observe that, since

$$
0=\left(D_{v_{\ell}} f\right)\left(\xi_{\ell}, u, \tilde{v}_{N}\left(\xi_{\ell}^{(N)}, u\right)\right)+\varepsilon_{N} \sum_{i=1}^{N} b_{1}\left(x_{0}, x_{\ell}, z_{i}\right) z_{i}, \quad 1 \leq \ell \leq N
$$

we get from Assumption Ai), for all $u, \tilde{u} \in U$,

$$
\begin{aligned}
0 & \left(\left(D_{v_{\ell}} f\right)\left(\xi_{\ell}, u, \tilde{v}_{N}\left(\xi_{\ell}^{(N)}, u\right)\right)-\left(D_{v_{\ell}} f\right)\left(\tilde{\xi}_{\ell}, \tilde{u}, \tilde{v}_{N}\left(\tilde{\xi}_{\ell}^{(N)}, \tilde{u}\right)\right), \tilde{v}_{N}\left(\xi_{\ell}^{(N)}, u\right)-\tilde{v}_{N}\left(\tilde{\xi}_{\ell}^{(N)}, \tilde{u}\right)\right) \\
& +\varepsilon_{N} \sum_{i=1}^{N}\left(b_{1}\left(x_{0}, x_{\ell}, z_{i}\right) z_{i}-b_{1}\left(\tilde{x}_{0}, \tilde{x}_{\ell}, \tilde{z}_{i}\right) \tilde{z}_{i}, \tilde{v}_{N}\left(\xi_{\ell}^{(N)}, u\right)-\tilde{v}_{N}\left(\tilde{\xi}_{\ell}^{(N)}, \tilde{u}\right)\right) \\
\geq \quad & \lambda\left|\tilde{v}_{N}\left(\xi_{\ell}^{(N)}, u\right)-\tilde{v}_{N}\left(\tilde{\xi}_{\ell}^{(N)}, \tilde{u}\right)\right|^{2} \\
& -\left|\left(D_{v_{l}} f\right)\left(\xi_{\ell}, u, \tilde{v}_{N}\left(\tilde{\xi}_{\ell}^{(N)}, \tilde{u}\right)\right)-\left(D_{v_{\ell}} f\right)\left(\tilde{\xi}_{\ell}, \tilde{u}, \tilde{v}_{N}\left(\tilde{\xi}_{\ell}^{(N)}, \tilde{u}\right)\right)\right|\left|\tilde{v}_{N}\left(\xi_{\ell}^{(N)}, u\right)-\tilde{v}_{N}\left(\tilde{\xi}_{\ell}^{(N)}, \tilde{u}\right)\right| \\
& -\varepsilon_{N} \sum_{i=1}^{N}\left|b_{1}\left(x_{0}, x_{\ell}, z_{i}\right) z_{i}-b_{1}\left(\tilde{x}_{0}, \tilde{x}_{\ell}, \tilde{z}_{i}\right) \tilde{z}_{i}\right|\left|\tilde{v}_{N}\left(\xi_{\ell}^{(N)}, u\right)-\tilde{v}_{N}\left(\tilde{\xi}_{\ell}^{(N)}, \tilde{u}\right)\right| \\
\geq & \lambda\left|\tilde{v}_{N}\left(\xi_{\ell}^{(N)}, u\right)-\tilde{v}_{N}\left(\tilde{\xi}_{\ell}^{(N)}, \tilde{u}\right)\right|^{2}-\left(C\left|\eta_{\ell}-\tilde{\eta}_{\ell}\right|+\mu|u-\tilde{u}|\right)\left|\tilde{v}_{N}\left(\xi_{\ell}^{(N)}, u\right)-\tilde{v}_{N}\left(\tilde{\xi}_{\ell}^{(N)}, \tilde{u}\right)\right| \\
& -\varepsilon_{N}\left\{N\left(\left|x_{0}-\tilde{x}_{0}\right|+\left|x_{\ell}-\tilde{x}_{\ell}\right|\right)+\sum_{i=1}^{N}\left|z_{i}-\tilde{z}_{i}\right|\right\}\left|\tilde{v}_{N}\left(\xi_{\ell}^{(N)}, u\right)-\tilde{v}_{N}\left(\tilde{\xi}_{\ell}^{(N)}, \tilde{u}\right)\right|,
\end{aligned}
$$

and, consequently,

$$
\begin{aligned}
\left|\tilde{v}_{N}\left(\xi_{\ell}^{(N)}, u\right)-\tilde{v}_{N}\left(\tilde{\xi}_{\ell}^{(N)}, \tilde{u}\right)\right| \leq & C\left|\eta_{\ell}-\tilde{\eta}_{\ell}\right|+\frac{\mu}{\lambda}|u-\tilde{u}|+C \varepsilon_{N} N\left(\left|x_{0}-\tilde{x}_{0}\right|+\left|x_{\ell}-\tilde{x}_{\ell}\right|\right) \\
& +C \varepsilon_{N} \sum_{i=1}^{N}\left|z_{i}-\tilde{z}_{i}\right|
\end{aligned}
$$

where $\eta_{\ell}:=\left(x_{0}, x_{\ell}, y, z_{0}\right), \tilde{\eta}_{\ell}:=\left(\tilde{x}_{0}, \tilde{x}_{\ell}, \tilde{y}, \tilde{z}_{0}\right)$. With a similar argument, using again Assumption Ai) but exploiting now the strict concavity of $f$ in $u$, we deduce from

$$
0=\frac{1}{N} \sum_{\ell=1}^{N}\left(D_{u} f\right)\left(\xi_{\ell}, \bar{u}^{N}\left(\xi^{(N)}\right), \bar{v}_{\ell}^{N}\left(\xi^{(N)}\right)\right)+\frac{1}{N} \sum_{\ell=1}^{N} b_{0}\left(x_{0}, x_{\ell}, z_{0}\right) z_{0}
$$

that

$$
\begin{aligned}
\left|\bar{u}^{N}\left(\xi^{(N)}\right)-\bar{u}^{N}\left(\tilde{\xi}^{(N)}\right)\right| \leq & \frac{\mu}{N \lambda} \sum_{\ell=1}^{N}\left|\bar{v}_{\ell}^{N}\left(\xi^{(N)}\right)-\bar{v}_{\ell}^{N}\left(\tilde{\xi}^{(N)}\right)\right| \\
& +C\left\{\left|x_{0}-\tilde{x}_{0}\right|+|y-\tilde{y}|+\left|z_{0}-\tilde{z}_{0}\right|+\frac{1}{N} \sum_{\ell=1}^{N}\left(\left|x_{\ell}-\tilde{x}_{\ell}\right|+\left|z_{\ell}-\tilde{z}_{\ell}\right|\right)\right\} .
\end{aligned}
$$

Hence, recalling that $\bar{v}_{\ell}^{N}\left(\xi^{(N)}\right)=\tilde{v}_{N}\left(\xi_{\ell}^{(N)}, \bar{u}^{N}\left(\xi^{(N)}\right)\right)$ and using (5.25) as well as $\mu / \lambda<1$, we obtain

$$
\begin{aligned}
& \left|\bar{u}^{N}\left(\xi^{(N)}\right)-\bar{u}^{N}\left(\tilde{\xi}^{(N)}\right)\right| \\
\leq & C\left(\left|x_{0}-\tilde{x}_{0}\right|+|y-\tilde{y}|+\left|z_{0}-\tilde{z}_{0}\right|\right)+C\left(1+\varepsilon_{N} N\right)\left\{\left|x_{0}-\tilde{x}_{0}\right|+\frac{1}{N} \sum_{\ell=1}^{N}\left(\left|x_{\ell}-\tilde{x}_{\ell}\right|+\left|z_{\ell}-\tilde{z}_{\ell}\right|\right)\right\},
\end{aligned}
$$

and combining the latter result with (5.25) we have

$$
\begin{aligned}
& \left|\bar{v}_{\ell}^{N}\left(\xi^{(N)}\right)-\bar{v}_{\ell}^{N}\left(\tilde{\xi}^{(N)}\right)\right|=\left|\tilde{v}_{N}\left(\xi_{\ell}^{(N)}, \bar{u}^{N}\left(\xi^{(N)}\right)\right)-\tilde{v}_{N}\left(\tilde{\xi}_{\ell}^{(N)}, \bar{u}^{N}\left(\tilde{\xi}^{(N)}\right)\right)\right| \\
\leq & C\left|\eta_{\ell}-\tilde{\eta}_{\ell}\right|+\frac{\mu}{\lambda}\left|\bar{u}^{N}\left(\xi^{(N)}\right)-\bar{u}^{N}\left(\tilde{\xi}^{(N)}\right)\right|+C \varepsilon_{N} N\left(\left|x_{0}-\tilde{x}_{0}\right|+\left|x_{\ell}-\tilde{x}_{\ell}\right|\right)+C \varepsilon_{N} \sum_{i=1}^{N}\left|z_{i}-\tilde{z}_{i}\right| \\
\leq & C\left|\eta_{\ell}-\tilde{\eta}_{\ell}\right|+C\left(1+\varepsilon_{N} N\right)\left(\left|x_{0}-\tilde{x}_{0}\right|+\left|x_{\ell}-\tilde{x}_{\ell}\right|+\frac{1}{N} \sum_{i=1}^{N}\left(\left|x_{i}-\tilde{x}_{i}\right|+\left|z_{i}-\tilde{z}_{i}\right|\right)\right) .
\end{aligned}
$$

With the relations (5.26) and (5.27) we have gotten the Lipschitz property stated for the saddle point control in the lemma. The proof is complete.

\subsection{Appendix 4}

Proof. (of Lemma 4.2). The statement 1) for $\bar{H}$ is a direct consequence of the estimates for $f$ in (3.6) and for $\bar{v}$ (see Lemma 4.1). In order to prove statement 2), it suffices to consider the function

$$
\bar{H}_{0}(s, \xi, u):=E\left[f_{\bar{v}}\left(\xi, \bar{X}_{s}^{1}, u\right) \mid \mathcal{F}_{T}^{W^{0}}\right], \quad(s, \xi, u) \in[0, T] \times \mathbb{R}^{3} \times U,
$$


and to determine its derivative with respect to $u$. For this note that, for all $h \in U$,

$$
\begin{aligned}
& \bar{H}_{0}(s, \xi, u+h)-\bar{H}_{0}(s, \xi, u)=E\left[\int_{0}^{1}\left(\left(D_{u} f_{\bar{v}}\right)\left(\xi, \bar{X}_{s}^{1}, u+\delta h\right), h\right) \mathrm{d} \delta \mid \mathcal{F}_{T}^{W^{0}}\right] \\
= & \left(E\left[\left(D_{u} f\right)\left(\xi, \bar{X}_{s}^{1}, u, \bar{v}\left(\xi, \bar{X}_{s}^{1}, u\right)\right) \mid \mathcal{F}_{T}^{W^{0}}\right], h\right) \\
& +E\left[\int_{0}^{1}\left(\left(D_{u} f\right)\left(\xi, \bar{X}_{s}^{1}, u+\delta h, \bar{v}\left(\xi, \bar{X}_{s}^{1}, u+\delta h\right)\right)-\left(D_{u} f\right)\left(\xi, \bar{X}_{s}^{1}, u, \bar{v}\left(\xi, \bar{X}_{s}^{1}, u\right)\right), h\right) \mathrm{d} \delta \mid \mathcal{F}_{T}^{W^{0}}\right] \\
= & \left(E\left[\left(D_{u} f\right)\left(\xi, \bar{X}_{s}^{1}, u, \bar{v}\left(\xi, \bar{X}_{s}^{1}, u\right)\right) \mid \mathcal{F}_{T}^{W^{0}}\right], h\right)+\rho(h),
\end{aligned}
$$

where, due to Lemma 4.1 ,

$$
|\rho(h)| \leq|h| E\left[\int_{0}^{1}\left(C|h|+\mu\left|\bar{v}\left(\xi, \bar{X}_{s}^{1}, u+\delta h\right)-\bar{v}\left(\xi, \bar{X}_{s}^{1}, u\right)\right|\right) \mathrm{d} \delta \mid \mathcal{F}_{T}^{W^{0}}\right] \leq C|h|^{2} .
$$

Hence,

$$
\left(D_{u} \bar{H}_{0}\right)(s, \xi, u)=E\left[\left(D_{u} f\right)\left(\xi, \bar{X}_{s}^{1}, u, \bar{v}\left(\xi, \bar{X}_{s}^{1}, u\right)\right) \mid \mathcal{F}_{T}^{W^{0}}\right],
$$

and statement 2) of the lemma follows.

Now by using this relation and the assumptions on $f$ and the Lipschitz property of $\bar{v}$ (Lemma 4.1), we get

$$
\begin{aligned}
& \left(\left(D_{u} \bar{H}\right)(s, \xi, u)-\left(D_{u} \bar{H}\right)\left(s, \xi, u^{\prime}\right), u-u^{\prime}\right)=\left(\left(D_{u} \bar{H}_{0}\right)(s, \xi, u)-\left(D_{u} \bar{H}_{0}\right)\left(s, \xi, u^{\prime}\right), u-u^{\prime}\right) \\
= & E\left[\left(\left(D_{u} f\right)\left(\xi, \bar{X}_{s}^{1}, u, \bar{v}\left(\xi, \bar{X}_{s}^{1}, u\right)\right)-\left(D_{u} f\right)\left(\xi, \bar{X}_{s}^{1}, u^{\prime}, \bar{v}\left(\xi, \bar{X}_{s}^{1}, u\right)\right), u-u^{\prime}\right) \mid \mathcal{F}_{T}^{W^{0}}\right] \\
& +E\left[\left(\left(D_{u} f\right)\left(\xi, \bar{X}_{s}^{1}, u^{\prime}, \bar{v}\left(\xi, \bar{X}_{s}^{1}, u\right)\right)-\left(D_{u} f\right)\left(\xi, \bar{X}_{s}^{1}, u^{\prime}, \bar{v}\left(\xi, \bar{X}_{s}^{1}, u^{\prime}\right)\right), u-u^{\prime}\right) \mid \mathcal{F}_{T}^{W^{0}}\right] \\
\leq & -\lambda\left|u-u^{\prime}\right|^{2}+\mu E\left[\left|\bar{v}\left(\xi, \bar{X}_{s}^{1}, u\right)-\bar{v}\left(\xi, \bar{X}_{s}^{1}, u^{\prime}\right)\right| \mid \mathcal{F}_{T}^{W^{0}}\right]\left|u-u^{\prime}\right| \\
\leq & -\lambda\left|u-u^{\prime}\right|^{2}+\frac{\mu^{2}}{\lambda}\left|u-u^{\prime}\right|^{2}=-\left(\lambda-\frac{\mu^{2}}{\lambda}\right)\left|u-u^{\prime}\right|^{2} .
\end{aligned}
$$

The proof is complete now.

We continue with the proof of Lemma 4.3 .

Proof. (of Lemma 4.3). From the definition of $\bar{u}(s, \xi)$ as the maximum point of the $C^{1}$-function $\bar{H}(s, \xi,$.$) :$ $U \rightarrow \mathbb{R}$ it follows that, for all $(s, \xi) \in[t, T] \times \mathbb{R}^{3}$,

$$
\begin{aligned}
0= & \left(\left(D_{u} \bar{H}\right)(s, \xi, \bar{u}(s, \xi)), \bar{u}(s, \xi)\right) \\
= & \left(\left(D_{u} \bar{H}\right)(s, \xi, \bar{u}(s, \xi))-\left(D_{u} \bar{H}\right)(s, \xi, 0), \bar{u}(s, \xi)\right) \\
& +\left(E\left[\left(D_{u} f\right)\left(\xi, \bar{X}_{s}^{1}, 0, \bar{v}\left(\xi, \bar{X}_{s}^{1}, 0\right)\right) \mid \mathcal{F}_{T}^{W^{0}}\right]+E\left[b_{0}\left(x_{0}, \bar{X}_{s}^{1}, z_{0}\right) z_{0} \mid \mathcal{F}_{T}^{W^{0}}\right], \bar{u}(s, \xi)\right) \\
\leq & -\left(\lambda-\frac{\mu^{2}}{\lambda}\right)|\bar{u}(s, \xi)|^{2}+C\left(1+E\left[\mu\left|\bar{v}\left(\xi, \bar{X}_{s}^{1}, 0\right)\right|\right]\right)|\bar{u}(s, \xi)| \\
\leq & -\left(\lambda-\frac{\mu^{2}}{\lambda}\right)|\bar{u}(s, \xi)|^{2}+C|\bar{u}(s, \xi)|,
\end{aligned}
$$

(see Lemmas 4.2 and 4.1), i.e.,

$$
|\bar{u}(s, \xi)| \leq C, \quad(s, \xi) \in[t, T] \times \mathbb{R}^{3} .
$$

For the same reason, using Lemmas 4.2 and 4.1 again, we also have

$$
\begin{aligned}
0 & \left(\left(D_{u} \bar{H}\right)(s, \xi, \bar{u}(s, \xi))-\left(D_{u} \bar{H}\right)\left(s, \xi^{\prime}, \bar{u}\left(s, \xi^{\prime}\right)\right), \bar{u}(s, \xi)-\bar{u}\left(s, \xi^{\prime}\right)\right) \\
\leq & -\left(\lambda-\frac{\mu^{2}}{\lambda}\right)\left|\bar{u}(s, \xi)-\bar{u}\left(s, \xi^{\prime}\right)\right|^{2}+\left(\left(D_{u} \bar{H}\right)\left(s, \xi, \bar{u}\left(s, \xi^{\prime}\right)\right)-\left(D_{u} \bar{H}\right)\left(s, \xi^{\prime}, \bar{u}\left(s, \xi^{\prime}\right)\right), \bar{u}(s, \xi)-\bar{u}\left(s, \xi^{\prime}\right)\right) \\
\leq & -\left(\lambda-\frac{\mu^{2}}{\lambda}\right)\left|\bar{u}(s, \xi)-\bar{u}\left(s, \xi^{\prime}\right)\right|^{2}+\left\{E \left[\mid\left(D_{u} f\right)\left(\xi, \bar{X}_{s}^{1}, \bar{u}\left(s, \xi^{\prime}\right), \bar{v}\left(\xi, \bar{X}_{s}^{1}, \bar{u}\left(s, \xi^{\prime}\right)\right)\right)\right.\right. \\
& \left.-\left(D_{u} f\right)\left(\xi^{\prime}, \bar{X}_{s}^{1}, \bar{u}\left(s, \xi^{\prime}\right), \bar{v}\left(\xi^{\prime}, \bar{X}_{s}^{1}, \bar{u}\left(s, \xi^{\prime}\right)\right)\right)|| \mathcal{F}_{T}^{W^{0}}\right] \\
& \left.+E\left[\left|b_{0}\left(x_{0}, \bar{X}_{s}^{1}, z_{0}\right) z_{0}-b_{0}\left(x_{0}^{\prime}, \bar{X}_{s}^{1}, z_{0}^{\prime}\right) z_{0}^{\prime}\right| \mid \mathcal{F}_{T}^{W^{0}}\right]\right\}\left|\bar{u}(s, \xi)-\bar{u}\left(s, \xi^{\prime}\right)\right| \\
\leq & -\left(\lambda-\frac{\mu^{2}}{\lambda}\right)\left|\bar{u}(s, \xi)-\bar{u}\left(s, \xi^{\prime}\right)\right|^{2} \\
& +C\left(\left|\xi-\xi^{\prime}\right|+E\left[\mu\left|\bar{v}\left(\xi, \bar{X}_{s}^{1}, \bar{u}\left(s, \xi^{\prime}\right)\right)-\bar{v}\left(\xi^{\prime}, \bar{X}_{s}^{1}, \bar{u}\left(s, \xi^{\prime}\right)\right)\right| \mid \mathcal{F}_{T}^{W^{0}}\right]\right)\left|\bar{u}(s, \xi)-\bar{u}\left(s, \xi^{\prime}\right)\right| \\
\leq & -\frac{1}{2}\left(\lambda-\frac{\mu^{2}}{\lambda}\right)\left|\bar{u}(s, \xi)-\bar{u}\left(s, \xi^{\prime}\right)\right|^{2}+C\left|\xi-\xi^{\prime}\right|^{2} .
\end{aligned}
$$

This proves the Lipschitz continuity of $\bar{u}(s,$.$) , uniformly with respect to s \in[0, T]$. 


\subsection{Appendix 5}

Let us begin with the proof of Lemma 4.5.

Proof. (of Lemma 4.5). Step 1. Recalling the definition of $\tilde{v}_{N}$ in (3.11) and that of $\bar{v}$ in (4.1), we see that

$$
\tilde{v}_{N}\left(x_{0}, x_{1}, y,\left(z_{0}, 0, \ldots, 0\right), u\right)=\bar{v}\left(x_{0}, x_{1}, y, z_{0}, u\right) .
$$

On the other hand, from (5.25),

$$
\left|\tilde{v}_{N}\left(x_{0}, x_{1}, y,\left(z_{0}, z_{1}, \ldots, z_{N}\right), u\right)-\tilde{v}_{N}\left(x_{0}, x_{1}, y,\left(z_{0}, 0, \ldots, 0\right), u\right)\right| \leq C \varepsilon_{N} \sum_{i=1}^{N}\left|z_{i}\right|,
$$

for all $\left(x_{0}, x_{1}, y, z_{0},\left(z_{1}, \ldots, z_{N}\right), u\right) \in \mathbb{R}^{N+4} \times U$. Consequently,

$$
\left|\tilde{v}_{N}\left(x_{0}, x_{1}, y, z^{(N)}, u\right)-\bar{v}\left(x_{0}, x_{1}, y, z_{0}, u\right)\right| \leq C \varepsilon_{N} \sum_{i=1}^{N}\left|z_{i}\right| .
$$

Step 2. The objective of this step is to estimate the difference between the controls $\bar{u}^{N}$ and $\bar{u}$.

Let us use here in our computations the notations $\xi:=\left(x_{0}, y, z_{0}\right)$ and $\left(\xi, x_{1}\right):=\left(x_{0}, x_{1}, y, z_{0}\right)$. Recall also the notations $\xi^{(N)}=\left(x^{(N)}, y, z^{(N)}\right)$ and $\xi^{(N, 0)}=\left(x^{(N)}, y,\left(z^{0, N}, 0, \ldots, 0\right)\right)$.

From the definition of $\bar{u}$ (see (4.8)) we get (see also (4.6):

$$
\begin{aligned}
0 & =\left(D_{u} \bar{H}\right)(s, \xi, \bar{u}(s, \xi)) \\
& =E\left[\left(D_{u} f\right)\left(\xi, \bar{X}_{s}^{1}, \bar{u}(s, \xi), \bar{v}\left(\xi, \bar{X}_{s}^{1}, \bar{u}(s, \xi)\right)\right)+b_{0}\left(x_{0}, \bar{X}_{s}^{1}, z_{0}\right) z_{0} \mid \mathcal{F}_{T}^{W^{0}}\right] .
\end{aligned}
$$

On the other hand, from the definition of $\bar{u}^{N}$ as one of the both saddle point feedback controls for $H_{N}\left(\xi^{(N)}, . ..\right)$, from Lemma 3.1 and from (5.28) we obtain

$$
\begin{aligned}
0= & \left(D_{u} H_{N}\right)\left(\xi^{(N, 0)}, \bar{u}^{N}\left(\xi^{(N, 0)}\right), \bar{v}^{N}\left(\xi^{(N, 0)}\right)\right) \\
= & \frac{1}{N} \sum_{\ell=1}^{N}\left(D_{u} f\right)\left(\xi, x_{\ell}, 0, \bar{u}^{N}\left(\xi^{(N, 0)}\right), \tilde{v}_{N}\left(\xi, x_{\ell}, 0, \bar{u}^{N}\left(\xi^{(N, 0)}\right)\right)\right) \\
& +\frac{1}{N} \sum_{\ell=1}^{N} b_{0}\left(x_{0}, x_{\ell}, z_{0}\right) z_{0} \\
= & \frac{1}{N} \sum_{\ell=1}^{N}\left(D_{u} f_{\bar{v}}\right)\left(\xi, x_{\ell}, 0, \bar{u}^{N}\left(\xi^{(N, 0)}\right)\right)+\frac{1}{N} \sum_{\ell=1}^{N} b_{0}\left(x_{0}, x_{\ell}, z_{0}\right) z_{0} .
\end{aligned}
$$

Let us use the notations $\bar{\Theta}_{s}=\left(X_{s}^{0}, \bar{Y}_{s}, \bar{Z}_{s}^{0}, 0\right), \bar{\Theta}_{s}^{N}=\left(X_{s}^{0}, \bar{Y}_{s}^{N}, \bar{Z}_{s}^{0, N}, 0\right)$, and, with abusing notation we also write $\left(\bar{\Theta}_{s}^{N}, X_{s}^{\ell}\right)=\left(X_{s}^{0}, X_{s}^{\ell}, \bar{Y}_{s}^{N}, \bar{Z}_{s}^{0, N}, 0\right)$. Moreover, let $\Xi_{s}^{(N)}=\left(X_{s}^{(N)}, \bar{Y}_{s}^{N}, \bar{Z}_{s}^{N}\right)$ and $\Xi_{s}^{(N, 0)}=$ $\left(X_{s}^{(N)}, \bar{Y}_{s}^{N},\left(\bar{Z}_{s}^{0, N}, 0, \ldots, 0\right)\right)$. Then subtracting (5.30) from (5.31) yields

$$
\begin{array}{rlr}
0= & \left(\left(\frac{1}{N} \sum_{\ell=1}^{N}\left(D_{u} f_{\bar{v}}\right)\left(\bar{\Theta}^{N}, X_{s}^{\ell}, \bar{u}^{N}\left(\Xi_{s}^{(N, 0)}\right)\right)+\frac{1}{N} \sum_{\ell=1}^{N} b_{0}\left(X_{s}^{0}, X_{s}^{\ell}, \bar{Z}_{s}^{0, N}\right) \bar{Z}_{s}^{0, N}\right)\right. \\
& \left.-E\left[\left(D_{u} f_{\bar{v}}\right)\left(\bar{\Theta}_{s}, \bar{X}_{s}^{1}, \bar{u}\left(s, \bar{\Theta}_{s}\right)\right)+b_{0}\left(X_{s}^{0}, \bar{X}_{s}^{1}, \bar{Z}_{s}^{0}\right) \bar{Z}_{s}^{0} \mid \mathcal{F}_{T}^{W^{0}}\right], \bar{u}^{N}\left(\Xi_{s}^{(N, 0)}\right)-\bar{u}\left(s, \bar{\Theta}_{s}\right)\right) & \\
= & \frac{1}{N} \sum_{\ell=1}^{N}\left(\left(D_{u} f_{\bar{v}}\right)\left(\bar{\Theta}_{s}^{N}, X_{s}^{\ell}, \bar{u}^{N}\left(\Xi_{s}^{(N, 0)}\right)\right)-\left(D_{u} f_{\bar{v}}\right)\left(\bar{\Theta}_{s}^{N}, X_{s}^{\ell}, \bar{u}^{(N,}\left(s, \bar{\Theta}_{s}\right)\right), \bar{u}^{N}\left(\Xi_{s}^{(N, 0)}\right)-\bar{u}\left(s, \bar{\Theta}_{s}\right)\right) & \left(=: I_{1}^{N}\right) \\
& +\frac{1}{N} \sum_{\ell=1}^{N}\left(\left(D_{u} f_{\bar{v}}\right)\left(\bar{\Theta}_{s}^{N}, X_{s}^{\ell}, \bar{u}\left(s, \bar{\Theta}_{s}\right)\right)-\left(D_{u} f_{\bar{v}}\right)\left(\bar{\Theta}_{s}, X_{s}^{\ell}, \bar{u}\left(s, \bar{\Theta}_{s}\right)\right), \bar{u}^{N}\left(\Xi_{s}^{(N, 0)}\right)-\bar{u}\left(s, \bar{\Theta}_{s}\right)\right) & \left(=: I_{2}^{N}\right) \\
& +\left(\frac{1}{N} \sum_{\ell=1}^{N} b_{0}\left(X_{s}^{0}, X_{s}^{\ell}, \bar{Z}_{s}^{0, N}\right) \bar{Z}_{s}^{0, N}-E\left[b_{0}\left(X_{s}^{0}, \bar{X}_{s}^{1}, \bar{Z}_{s}^{0}\right) \bar{Z}_{s}^{0} \mid \mathcal{F}_{T}^{W^{0}}\right], \bar{u}^{N}\left(\Xi_{s}^{(N, 0)}\right)-\bar{u}\left(s, \bar{\Theta}_{s}\right)\right) & \left(=: I_{3}^{N}\right) \\
& +\left(\frac{1}{N} \sum_{\ell=1}^{N}\left(D_{u} f_{\bar{v}}\right)\left(\bar{\Theta}_{s}, X_{s}^{\ell}, \bar{u}\left(s, \bar{\Theta}_{s}\right)\right)-E\left[\left(D_{u} f_{\bar{v}}\right)\left(\bar{\Theta}_{s}, \bar{X}_{s}^{1}, \bar{u}\left(s, \bar{\Theta}_{s}\right)\right) \mid \mathcal{F}_{T}^{W^{0}}\right], \bar{u}^{N}\left(\Xi_{s}^{(N, 0)}\right)-\bar{u}\left(s, \bar{\Theta}_{s}\right)\right) \cdot\left(=: I_{4}^{N}\right)
\end{array}
$$

Let us estimate the expressions $I_{k}^{N}, 1 \leq k \leq 4$. We begin with that of $I_{1}^{N}$.

- Estimate for $I_{1}^{N}$ : By standard estimates using our assumptions on $f$ we have

$$
\begin{aligned}
& =\left\{\begin{array}{l}
\left.\left(D_{u} f_{\bar{v}}\right)\left(\xi, x_{1}, 0, u\right)-\left(D_{u} f_{\bar{v}}\right)\left(\xi, x_{1}, 0, u^{\prime}\right), u-u^{\prime}\right) \\
\left.\left(D_{u} f\right)\left(\xi, x_{1}, 0, u, \bar{v}\left(\xi, x_{1}, 0, u\right)\right)-\left(D_{u} f\right)\left(\xi, x_{1}, 0, u^{\prime}, \bar{v}\left(\xi, x_{1}, 0, u\right)\right), u-u^{\prime}\right)
\end{array}\right. \\
& +\left(\left(D_{u} f\right)\left(\xi, x_{1}, 0, u^{\prime}, \bar{v}\left(\xi, x_{1}, 0, u\right)\right)-\left(D_{u} f\right)\left(\xi, x_{1}, 0, u^{\prime}, \bar{v}\left(\xi, x_{1}, 0, u^{\prime}\right)\right), u-u^{\prime}\right) \\
& \leq-\lambda\left|u-u^{\prime}\right|^{2}+\mu\left|\bar{v}\left(\xi, x_{1}, 0, u\right)-\bar{v}\left(\xi, x_{1}, 0, u^{\prime}\right)\right|\left|u-u^{\prime}\right| \\
& \leq-\left(\lambda-\frac{\mu^{2}}{\lambda}\right)\left|u-u^{\prime}\right|^{2}
\end{aligned}
$$


(recall that we have supposed that $\mu<\lambda$ ). Consequently,

$$
I_{1}^{N} \leq-\left(\lambda-\frac{\mu^{2}}{\lambda}\right)\left|\bar{u}^{N}\left(\Xi_{s}^{(N, 0)}\right)-\bar{u}\left(s, \bar{\Theta}_{s}\right)\right|^{2} .
$$

- Estimate for $I_{2}^{N}$ : From (4.3) and Assumption Aii) on $D_{u} f$ we have, for arbitrarily small given $\delta>0$ and a constant $C_{\delta}$ only depending on $\delta$,

$$
\begin{aligned}
I_{2}^{N} & \leq C\left|\bar{\Theta}_{s}^{N}-\bar{\Theta}_{s}\right|\left|\bar{u}^{N}\left(\Xi_{s}^{(N, 0)}\right)-\bar{u}\left(s, \bar{\Theta}_{s}\right)\right| \\
& \leq C_{\delta}\left(\left|\bar{Y}_{s}^{N}-\bar{Y}_{s}\right|^{2}+\left|\bar{Z}_{s}^{0, N}-\bar{Z}_{s}^{0}\right|^{2}\right)+\delta\left|\bar{u}^{N}\left(\Xi_{s}^{(N, 0)}\right)-\bar{u}\left(s, \bar{\Theta}_{s}\right)\right|^{2} .
\end{aligned}
$$

- Estimate for $I_{3}^{N}$ : Using the Lipschitz continuity of $z_{0} \mapsto b_{0}\left(x_{0}, x_{1}, z_{0}\right) z_{0}$, uniformly with respect to $\left(x_{0}, x_{1}\right)$, we obtain that for any small $\delta>0$ there is a constant $C_{\delta}$ such that

$$
\begin{aligned}
I_{3}^{N} \leq & C_{\delta}\left|\bar{Z}_{s}^{0, N}-\bar{Z}_{s}^{0}\right|^{2}+\delta\left|\bar{u}^{N}\left(\Xi_{s}^{(N, 0)}\right)-\bar{u}\left(s, \bar{\Theta}_{s}\right)\right|^{2} \\
& +C_{\delta}\left|\frac{1}{N} \sum_{\ell=1}^{N} b_{0}\left(X_{s}^{0}, X_{s}^{\ell}, \bar{Z}_{s}^{0}\right) \bar{Z}_{s}^{0}-E\left[b_{0}\left(X_{s}^{0}, \bar{X}_{s}^{1}, \bar{Z}_{s}^{0}\right) \bar{Z}_{s}^{0} \mid \mathcal{F}_{T}^{W^{0}}\right]\right|^{2} .
\end{aligned}
$$

Recalling that $\left|b_{0}\left(x_{0}, x_{1}, z_{0}\right) z_{0}-b_{0}\left(x_{0}, x_{1}^{\prime}, z_{0}\right) z_{0}\right| \leq C\left|x_{1}-x_{1}^{\prime}\right|$, we can use (2.14), in order to deduce that for all $m \geq 1$, there is some constant $C_{m}$ such that

$$
E\left[\left|\frac{1}{N} \sum_{\ell=1}^{N} b_{0}\left(x_{0}, X_{s}^{\ell}, z_{0}\right) z_{0}-E\left[b_{0}\left(x_{0}, \bar{X}_{s}^{1}, z_{0}\right) z_{0} \mid \mathcal{F}_{T}^{W^{0}}\right]\right|^{2 m} \mid \mathcal{F}_{T}^{W^{0}}\right] \leq C_{m}\left(\frac{1}{N}+\frac{1}{N} \sum_{\ell=1}^{N}\left|x_{\ell}-\bar{x}\right|^{2}\right)^{m},
$$

for all $N \geq 1, s \in[t, T],\left(x_{0}, z_{0}\right) \in \mathbb{R}^{2}$. Hence, as $X_{s}^{0}$ and $\bar{Z}_{s}^{0}$ are $\mathcal{F}_{s}^{W^{0}}$-measurable,

$$
\begin{aligned}
& E\left[\left|\frac{1}{N} \sum_{\ell=1}^{N} b_{0}\left(X_{s}^{0}, X_{s}^{\ell}, \bar{Z}_{s}^{0}\right) \bar{Z}_{s}^{0}-E\left[b_{0}\left(X_{s}^{0}, \bar{X}_{s}^{1}, \bar{Z}_{s}^{0}\right) \bar{Z}_{s}^{0} \mid \mathcal{F}_{T}^{W^{0}}\right]\right|^{2 m} \mid \mathcal{F}_{T}^{W^{0}}\right] \\
& \leq C_{m}\left(\frac{1}{N}+\frac{1}{N} \sum_{\ell=1}^{N}\left|x_{\ell}-\bar{x}\right|^{2}\right)^{m} .
\end{aligned}
$$

- Estimate for $I_{4}^{N}$ : Obviously, for all $\delta>0$ there is $C_{\delta}>0$ such that

$$
\begin{aligned}
I_{4}^{N} \leq & \delta\left|\bar{u}^{N}\left(\Xi_{s}^{(N, 0)}\right)-\bar{u}\left(s, \bar{\Theta}_{s}\right)\right|^{2} \\
& +C_{\delta}\left|\frac{1}{N} \sum_{\ell=1}^{N}\left(D_{u} f_{\bar{v}}\right)\left(\bar{\Theta}_{s}, X_{s}^{\ell}, \bar{u}\left(s, \bar{\Theta}_{s}\right)\right)-E\left[\left(D_{u} f_{\bar{v}}\right)\left(\bar{\Theta}_{s}, \bar{X}_{s}^{1}, \bar{u}\left(s, \bar{\Theta}_{s}\right)\right) \mid \mathcal{F}_{T}^{W^{0}}\right]\right|^{2} .
\end{aligned}
$$

Noting that $\left|\left(D_{u} f_{\bar{v}}\right)\left(\xi, x_{1}, 0, u\right)-\left(D_{u} f_{\bar{v}}\right)\left(\xi, x_{1}^{\prime}, 0, u\right)\right| \leq C\left|x_{1}-x_{1}^{\prime}\right|$ and observing that $\bar{\Theta}_{s}$ is $\mathcal{F}_{s}^{W^{0}}$-measurable, we obtain similarly to the estimate for $I_{3}^{N}$ from (2.14) that, for all $m \geq 1$ there is some $C_{m} \in \mathbb{R}$ with

$$
\begin{aligned}
& E\left[\left|\frac{1}{N} \sum_{\ell=1}^{N}\left(D_{u} f_{\bar{v}}\right)\left(\bar{\Theta}_{s}, X_{s}^{\ell}, \bar{u}\left(s, \bar{\Theta}_{s}\right)\right)-E\left[\left(D_{u} f_{\bar{v}}\right)\left(\bar{\Theta}_{s}, \bar{X}_{s}^{1}, \bar{u}\left(s, \bar{\Theta}_{s}\right)\right) \mid \mathcal{F}_{T}^{W^{0}}\right]\right|^{2 m} \mid \mathcal{F}_{T}^{W^{0}}\right] \\
\leq & C_{m}\left(\frac{1}{N}+\frac{1}{N} \sum_{\ell=1}^{N}\left|x_{\ell}-\bar{x}\right|^{2}\right)^{m} .
\end{aligned}
$$

Now, choosing $\delta=\frac{1}{6}\left(\lambda-\frac{\mu^{2}}{\lambda}\right)>0$, and combing the above estimates for $I_{k}^{N}, 1 \leq k \leq 4$, we obtain

$$
\begin{aligned}
0= & I_{1}^{N}+I_{2}^{N}+I_{3}^{N}+I_{4}^{N} \\
\leq & -3 \delta\left|\bar{u}^{N}\left(\Xi_{s}^{(N, 0)}\right)-\bar{u}\left(s, \bar{\Theta}_{s}\right)\right|^{2} \\
& +C\left(\left|\bar{Y}_{s}^{N}-\bar{Y}_{s}\right|^{2}+\left|\bar{Z}_{s}^{0, N}-\bar{Z}_{s}^{0}\right|^{2}\right)+\left|R^{N}\left(s, \bar{\Theta}_{s}, \bar{u}\left(s, \bar{\Theta}_{s}\right)\right)\right|^{2}
\end{aligned}
$$

where

$$
\begin{aligned}
R^{N}(s, \xi, u):= & C \frac{1}{N} \sum_{\ell=1}^{N}\left|\left(D_{u} f_{\bar{v}}\right)\left(\xi, X_{s}^{\ell}, u\right)-E\left[\left(D_{u} f_{\bar{v}}\right)\left(\xi, \bar{X}_{s}^{\ell}, u\right) \mid \mathcal{F}_{T}^{W^{0}}\right]\right| \\
& +C \frac{1}{N} \sum_{\ell=1}^{N}\left|b_{0}\left(x_{0}, X_{s}^{\ell}, z_{0}\right) z_{0}-E\left[b_{0}\left(x_{0}, \bar{X}_{s}^{1}, z_{0}\right) z_{0} \mid \mathcal{F}_{T}^{W^{0}}\right]\right|
\end{aligned}
$$

and

$$
E\left[\left|R^{N}\left(s, \bar{\Theta}_{s}, \bar{u}\left(s, \bar{\Theta}_{s}\right)\right)\right|^{2 m} \mid \mathcal{F}_{T}^{W^{0}}\right] \leq C_{m}\left(\frac{1}{N}+\frac{1}{N} \sum_{\ell=1}^{N}\left|x_{\ell}-\bar{x}\right|^{2}\right)^{m}
$$


We recall that $\bar{u}_{s}^{N}=\bar{u}^{N}\left(\Xi_{s}^{(N)}\right)\left(=\bar{u}^{N}\left(X_{s}^{(N)}, \bar{Y}_{s}^{N}, \bar{Z}^{(N)}\right)\right)$, and we put $\bar{u}_{s}^{N, 0}=\bar{u}^{N}\left(\Xi_{s}^{(N, 0)}\right)\left(=\bar{u}^{N}\left(X_{s}^{(N)}, \bar{Y}_{s}^{N}\right.\right.$, $\left.\left.\left(\bar{Z}^{0, N}, 0, \ldots, 0\right)\right)\right)$ and $\bar{u}_{s}=\bar{u}\left(s, \bar{\Theta}_{s}\right)\left(=\bar{u}\left(s, X_{s}^{0}, \bar{Y}_{s}, \bar{Z}_{s}^{0}\right)\right)$.

Then, taking into account that by Lemma 3.2 (iii) we have

$$
\left|\bar{u}_{s}^{N}-\bar{u}_{s}^{N, 0}\right| \leq C\left(1+\varepsilon_{N} \cdot N\right) \frac{1}{N} \sum_{l=\ell}^{N}\left|\bar{Z}_{s}^{\ell, N}\right|,
$$

we obtain from (5.38)

$$
\begin{aligned}
& \left|\bar{u}_{s}^{N}-\bar{u}_{s}\right| \leq\left|\bar{u}_{s}^{N}-\bar{u}_{s}^{N, 0}\right|+\left|\bar{u}_{s}^{N, 0}-\bar{u}_{s}\right| \\
\leq & C\left(\left|\bar{Y}_{s}^{N}-\bar{Y}_{s}\right|+\left|\bar{Z}_{s}^{0, N}-\bar{Z}_{s}^{0}\right|+\left(1+\varepsilon_{N} \cdot N\right) \frac{1}{N} \sum_{\ell=1}^{N}\left|\bar{Z}_{s}^{\ell, N}\right|\right)+C\left|R^{N}\left(s, \bar{\Theta}_{s}, \bar{u}_{s}\right)\right|,
\end{aligned}
$$

where

$$
E\left[\left.R^{N}\left(s, \bar{\Theta}_{s}, \bar{u}_{s}\right)\right|^{2 m} \mid \mathcal{F}_{T}^{W^{0}}\right] \leq C_{m}\left(\frac{1}{N}+\frac{1}{N} \sum_{\ell=1}^{N}\left|x_{\ell}-\bar{x}\right|^{2}\right)^{m} .
$$

Step 3. Basing on the results of above steps we prove now the limit behavior of the controls process $\bar{v}_{s}^{(N)}=$

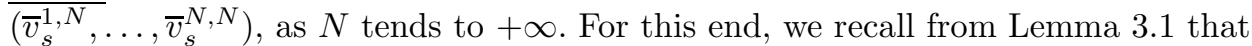

$$
\bar{v}_{s}^{\ell, N}=\bar{v}_{\ell}^{N}\left(X_{s}^{(N)}, \bar{Y}_{s}^{N}, \bar{Z}_{s}^{(N)}\right)=\tilde{v}_{N}\left(X_{s}^{0}, X_{s}^{\ell}, \bar{Y}_{s}^{N}, \bar{Z}_{s}^{(N)}, \bar{u}_{s}\right), s \in[t, T], 1 \leq \ell \leq N .
$$

Thus, due to the estimates (5.29) in Step 1 and (5.40) in Step 2 as well as Lemma 4.1 we have

$$
\begin{aligned}
& \left|\bar{v}_{s}^{\ell, N}-\bar{v}_{s}^{\ell}\right| \\
\leq & \left|\widetilde{v}_{N}\left(X_{s}^{0}, X_{s}^{\ell}, \bar{Y}_{s}^{N}, \bar{Z}_{s}^{(N)}, \bar{u}_{s}^{N}\right)-\bar{v}\left(X_{s}^{0}, X_{s}^{\ell}, \bar{Y}_{s}^{N}, \bar{Z}_{s}^{0, N}, \bar{u}_{s}^{N}\right)\right| \\
& +\left|\bar{v}\left(X_{s}^{0}, X_{s}^{\ell}, \bar{Y}_{s}^{N}, \bar{Z}_{s}^{0, N}, \bar{u}_{s}^{N}\right)-\bar{v}\left(X_{s}^{0}, X_{s}^{\ell}, \bar{Y}_{s}, \bar{Z}_{s}^{0}, \bar{u}_{s}\right)\right| \\
\leq & C \varepsilon_{N} \sum_{\ell=1}^{N}\left|\bar{Z}_{s}^{\ell, N}\right|+C\left(\left|\bar{Y}_{s}^{N}-\bar{Y}_{s}\right|+\left|\bar{Z}_{s}^{0, N}-\bar{Z}_{s}^{0}\right|+\left|\bar{u}_{s}^{N}-\bar{u}_{s}\right|\right) \\
\leq & C\left(\left|\bar{Y}_{s}^{N}-\bar{Y}_{s}\right|+\left|\bar{Z}_{s}^{0, N}-\bar{Z}_{s}^{0}\right|+\left(1+\varepsilon_{N} \cdot N\right) \frac{1}{N} \sum_{\ell=1}^{N}\left|\bar{Z}_{s}^{\ell, N}\right|\right)+C\left|R^{N}\left(s, \bar{\Theta}_{s}, \bar{u}_{s}\right)\right|
\end{aligned}
$$

We recall that an estimate for $R^{N}\left(s, \bar{\Theta}_{s}, \bar{u}_{s}\right)$ is given in (5.40) in Step 2. The proof is complete now.

Let us come, finally, to the proof of Lemma 4.6.

Proof. (of Lemma 4.6). Let us keep notations introduced in the preceding proof. So we recall that, in particular, $\bar{u}_{s}^{N, 0}=\bar{u}^{N}\left(\Xi_{s}^{(N, 0)}\right)\left(=\bar{u}^{N}\left(X_{s}^{(N)}, \bar{Y}_{s}^{N},\left(\bar{Z}^{0, N}, 0, \ldots, 0\right)\right)\right)$, and we introduce in the same sense the notation $\bar{v}_{s}^{\ell, N, 0}=\bar{v}_{\ell}^{N}\left(X_{s}^{(N)}, \bar{Y}_{s}^{N},\left(\bar{Z}_{s}^{0, N}, 0, \ldots, 0\right)\right)$. Then, using our assumptions on $f$ (see (3.6) $)$ we get

$$
\begin{aligned}
& \left|f\left(X_{s}^{0}, X_{s}^{\ell}, \bar{Y}_{s}^{N}, \bar{Z}_{s}^{0, N}, 0, \bar{u}_{s}^{N, 0}, \bar{v}_{s}^{\ell, N, 0}\right)-f\left(X_{s}^{0}, X_{s}^{\ell}, \bar{Y}_{s}, \bar{Z}_{s}^{0}, 0, \bar{u}_{s}, \bar{v}_{s}^{\ell}\right)\right| \\
\leq & C\left(\left|\bar{Y}_{s}^{N}-\bar{Y}_{s}\right|+\left|\bar{Z}_{s}^{0, N}-\bar{Z}_{s}^{0}\right|+\left|\bar{u}_{s}^{N, 0}-\bar{u}_{s}\right|+\left|\bar{v}_{s}^{\ell, N, 0}-\bar{v}_{s}^{\ell}\right|\right) .
\end{aligned}
$$

Indeed, from (3.15) of Lemma 3.2 we know that the processes $\bar{u}^{N, 0}$ and $\bar{v}^{\ell, N, 0}$ are bounded by a constant not depending on $N$. On the other hand, from Lemma 4.3 we have the boundedness of process $\bar{u}$, and from the Lemmas 4.3 and 4.1 we obtain also that of the process $\bar{v}^{\ell}$.

Moreover, from Lemma 4.5 it follows that

$$
\left|\bar{u}_{s}^{N, 0}-\bar{u}_{s}\right|+\left|\bar{v}_{s}^{\ell, N, 0}-\bar{v}_{s}^{\ell}\right| \leq C\left(\left|\bar{Y}_{s}^{N}-\bar{Y}_{s}\right|+\left|\bar{Z}_{s}^{0, N}-\bar{Z}_{s}^{0}\right|\right)+R_{s}^{N} .
$$

Hence,

$$
\begin{aligned}
& \left|f\left(X_{s}^{0}, X_{s}^{\ell}, \bar{Y}_{s}^{N}, \bar{Z}_{s}^{0, N}, 0, \bar{u}_{s}^{N, 0}, \bar{v}_{s}^{\ell, N, 0}\right)-f\left(X_{s}^{0}, X_{s}^{\ell}, \bar{Y}_{s}, \bar{Z}_{s}^{0}, 0, \bar{u}_{s}, \bar{v}_{s}^{\ell}\right)\right| \\
\leq & C\left(\left|\bar{Y}_{s}^{N}-\bar{Y}_{s}\right|+\left|\bar{Z}_{s}^{0, N}-\bar{Z}_{s}^{0}\right|\right)+R_{s}^{N},
\end{aligned}
$$


with $R_{s}^{N}$ satisfying the estimate given in Lemma 4.5. We also note that, by using the assumptions on $b_{0}$, we can show with similar arguments that

$$
\begin{aligned}
& \left|b_{0}\left(X_{s}^{0}, X_{s}^{\ell}, \bar{Z}_{s}^{0, N}\right) \bar{Z}_{s}^{0, N} \bar{u}_{s}^{N, 0}-b_{0}\left(X_{s}^{0}, X_{s}^{\ell}, \bar{Z}_{s}^{0}\right) \bar{Z}_{s}^{0} \bar{u}_{s}\right| \\
\leq & C\left|\bar{Z}_{s}^{0, N}-\bar{Z}_{s}^{0}\right|+C\left|\bar{u}_{s}^{N, 0}-\bar{u}_{s}\right| \\
\leq & C\left(\left|\bar{Y}_{s}^{N}-\bar{Y}_{s}^{0}\right|+\left|\bar{Z}_{s}^{0, N}-\bar{Z}_{s}^{0}\right|\right)+R_{s}^{N}, s \in[t, T], N \geq 1 .
\end{aligned}
$$

Consequently, recalling the notations introduced for this proof and for the preceding one, and by using the fact that $\left(\bar{u}^{N}\left(\xi^{(N)}\right), \bar{v}^{N}\left(\xi^{(N)}\right)\right)$ is a saddle point of the Hamiltonian $H_{N}\left(\xi^{(N)}, \cdot, \cdot\right)$ and $\left(\bar{u}^{N}\left(\xi^{(N, 0)}\right), \bar{v}^{N}\left(\xi^{(N, 0)}\right)\right)$ is one of $H_{N}\left(\xi^{(N, 0)}, \cdot, \cdot\right)\left(=H_{N}\left(x^{(N)}, y,\left(z^{0}, 0, \ldots, 0, . ..\right)\right)\right)$, we observe

$$
\begin{aligned}
& \bar{H}_{N}\left(\Xi_{s}^{(N)}\right):=H_{N}\left(\Xi_{s}^{(N)}, \bar{u}_{s}^{N}, \bar{v}_{s}^{N}\right) \leq H_{N}\left(\Xi_{s}^{(N)}, \bar{u}_{s}^{N}, \bar{v}_{s}^{N, 0}\right) \\
= & H_{N}\left(\Xi_{s}^{(N, 0)}, \bar{u}_{s}^{N}, \bar{v}_{s}^{N, 0}\right)+\frac{1}{N} \sum_{\ell=1}^{N}\left(f\left(X_{s}^{0}, X_{s}^{\ell}, \bar{Y}_{s}^{N}, \bar{Z}_{s}^{0, N}, \bar{Z}_{s}^{\ell, N}, \bar{u}_{s}^{N}, \bar{v}_{s}^{\ell, N, 0}\right)\right. \\
& \left.-f\left(X_{s}^{0}, X_{s}^{\ell}, \bar{Y}_{s}^{N}, \bar{Z}_{s}^{0, N}, 0, \bar{u}_{s}^{N}, \bar{v}_{s}^{\ell, N, 0}\right)\right)+\varepsilon_{N} \frac{1}{N} \sum_{\ell=1}^{N}\left(\sum_{i=1}^{N} b_{1}\left(X_{s}^{0}, X_{s}^{\ell}, \bar{Z}_{s}^{i, N}\right) \bar{Z}_{s}^{i, N}\right) \bar{v}_{s}^{\ell, N, 0} \\
\leq & H_{N}\left(\Xi_{s}^{(N, 0)}, \bar{u}_{s}^{N}, \bar{v}_{s}^{N, 0}\right)+C\left(\frac{1}{N}+\varepsilon_{N}\right) \sum_{\ell=1}^{N}\left|\bar{Z}_{s}^{\ell, N}\right| \\
\leq & H_{N}\left(\Xi_{s}^{(N, 0)}, \bar{u}_{s}^{N, 0}, \bar{v}_{s}^{N, 0}\right)+C\left(\frac{1}{N}+\varepsilon_{N}\right) \sum_{\ell=1}^{N}\left|\bar{Z}_{s}^{\ell, N}\right| .
\end{aligned}
$$

On the other hand, estimating $\bar{H}_{N}\left(\Xi_{s}^{(N)}\right)$ in the opposite direction by using similar arguments as above and the fact that $\left|\bar{v}_{s}^{\ell, N}\right| \leq C\left(1+N \varepsilon_{N}\right)$ (see Lemma 3.2), we obtain

$$
\begin{aligned}
& \bar{H}_{N}\left(\Xi_{s}^{(N)}\right)=H_{N}\left(\Xi_{s}^{(N)}, \bar{u}_{s}^{N}, \bar{v}_{s}^{N}\right) \geq H_{N}\left(\Xi_{s}^{N}, \bar{u}_{s}^{N, 0}, \bar{v}_{s}^{N}\right) \\
= & H_{N}\left(\Xi_{s}^{(N, 0)}, \bar{u}_{s}^{N, 0}, \bar{v}_{s}^{N}\right)+\frac{1}{N} \sum_{\ell=1}^{N}\left(f\left(X_{s}^{0}, X_{s}^{\ell}, \bar{Y}_{s}^{N}, \bar{Z}_{s}^{0, N}, \bar{Z}_{s}^{\ell, N}, \bar{u}_{s}^{N, 0}, \bar{v}_{s}^{\ell, N}\right)\right. \\
& -f\left(X_{s}^{0}, X_{s}^{\ell}, \bar{Y}_{s}^{N}, \bar{Z}_{s}^{0, N}, 0, \bar{u}_{s}^{N, 0}, \bar{v}_{s}^{\ell, N}\right)+\varepsilon_{N} \frac{1}{N} \sum_{\ell=1}^{N}\left(\sum_{i=1}^{N} b_{1}\left(X_{s}^{0}, X_{s}^{\ell}, \bar{Z}_{s}^{i, N}\right) \bar{Z}_{s}^{i, N}\right) \bar{v}_{s}^{\ell, N} \\
\geq & H_{N}\left(\Xi_{s}^{(N, 0)}, \bar{u}_{s}^{N, 0}, \bar{v}_{s}^{N}\right)-C \frac{1}{N} \sum_{\ell=1}^{N}\left|\bar{Z}_{s}^{\ell, N}\right|-C \varepsilon_{N}\left(1+N \varepsilon_{N}\right) \sum_{i=1}^{N}\left|\bar{Z}_{s}^{i, N}\right| \\
\geq & H_{N}\left(\Xi_{s}^{(N, 0)}, \bar{u}_{s}^{N, 0}, \bar{v}_{s}^{N}\right)-C\left(\frac{1}{N}+\varepsilon_{N}+N \varepsilon_{N}^{2}\right) \sum_{\ell=1}^{N}\left|\bar{Z}_{s}^{\ell, N}\right| \\
\geq & H_{N}\left(\Xi_{s}^{(N, 0)}, \bar{u}_{s}^{N, 0}, \bar{v}_{s}^{N, 0}\right)-C\left(\frac{1}{N}+\varepsilon_{N}+N \varepsilon_{N}^{2}\right) \sum_{\ell=1}^{N}\left|\bar{Z}_{s}^{\ell, N}\right| .
\end{aligned}
$$

By combining (5.45) and (5.46) we obtain

$$
\left|\bar{H}_{N}\left(\Xi_{s}^{(N)}\right)-\bar{H}_{N}\left(\Xi_{s}^{(N, 0)}\right)\right| \leq C\left(\frac{1}{N}+\varepsilon_{N}+N \varepsilon_{N}^{2}\right) \sum_{\ell=1}^{N}\left|\bar{Z}_{s}^{\ell, N}\right| .
$$

Finally, from (5.43) and (5.44) we see

$$
\begin{aligned}
& \left|\bar{H}_{N}\left(\Xi_{s}^{(N, 0)}\right)-\left(\frac{1}{N} \sum_{\ell=1}^{N} f\left(X_{s}^{0}, X_{s}^{\ell}, \bar{Y}_{s}, \bar{Z}_{s}^{0}, 0, \bar{u}_{s}, \bar{v}_{s}^{\ell}\right)+\frac{1}{N} \sum_{\ell=1}^{N} b_{0}\left(X_{s}^{0}, X_{s}^{\ell}, \bar{Z}_{s}^{0}\right) \bar{Z}_{s}^{0} \bar{u}_{s}\right)\right| \\
\leq & C\left(\left|\bar{Y}_{s}^{N}-\bar{Y}_{s}\right|+\left|\bar{Z}_{s}^{0, N}-\bar{Z}_{s}^{0}\right|\right)+R_{s}^{N}, \quad s \in[t, T], \quad N \geq 1 .
\end{aligned}
$$

Recalling that the functions $x_{\ell} \rightarrow f\left(x_{0}, x_{l}, y, z_{0}, u\right)$, and $x_{l} \rightarrow b_{0}\left(x_{0}, x_{l}, z_{0}\right) z_{0}$ are Lipschitz, uniformly with respect to $\left(x_{0}, y, z_{0}\right)$, and that $\left|\bar{u}_{s}\right| \leq C$, we can apply Lemma 2.1. and we get for

$$
\begin{aligned}
& \bar{R}_{1}^{N}\left(s, x_{0}, y, z_{0}, u\right):=\left|\frac{1}{N} \sum_{\ell=1}^{N} f\left(x_{0}, X_{s}^{\ell}, y, z_{0}, 0, u, \bar{v}_{s}^{\ell}\right)-E\left[f\left(x_{0}, \bar{X}_{s}^{1}, y, z_{0}, 0, u, \bar{v}_{s}^{\ell}\right) \mid \mathcal{F}_{T}^{W^{0}}\right]\right| ; \\
& \bar{R}_{2}^{N}\left(s, x_{0}, z_{0}\right):=\left|\frac{1}{N} \sum_{\ell=1}^{N} b_{0}\left(x_{0}, X_{s}^{\ell}, z_{0}\right) z_{0}-E\left[b_{0}\left(x_{0}, \bar{X}_{s}^{1}, z_{0}\right) z_{0} \mid \mathcal{F}_{T}^{W^{0}}\right]\right|, \\
& \bar{R}^{N}\left(s, x_{0}, y, z_{0}, u\right):=\bar{R}_{1}^{N}\left(s, x_{0}, y, z_{0}, u\right)+\bar{R}_{2}^{N}\left(s, x_{0}, z_{0}\right) u
\end{aligned}
$$

the estimate

$$
E\left[\left|\bar{R}^{N}\left(s, X_{s}^{0}, \bar{Y}_{s}, \bar{Z}_{s}^{0}, \bar{u}_{s}\right)\right|^{2 m} \mid \mathcal{F}_{T}^{W^{0}}\right] \leq C_{m}\left(\frac{1}{N}+\frac{1}{N} \sum_{\ell=1}^{N}\left|x_{\ell}-\bar{x}\right|^{2}\right)^{m}, \text { where } s \in[t, T], N \geq 1, m \geq 1 .
$$

The statement of the lemma follows now easily from the latter estimates and (5.48). 


\section{References}

[1] BENSOUSSAN, A., SUNG, K.C.J., Yam, S.C.P. and YUNG, S.P. (2011) Linear quadratic mean field games, Technical Report.

[2] BUCKDAHN, R., LI, J. and PENG, S. (2009) Mean-field backward stochastic differential equations and related partial differential equations, Stochstic Processes and their Applications, 119, 3133-3154.

[3] BUCKDAHN, R., DJEHICHE, B., LI, J. and PENG, S. (2009) Mean-field backward stochastic differential equations. A limit approach, The Annals of Probability, 37, 1524-1565.

[4] CARDAliaguet, P. (2012) Notes on Mean Field Games, (from P.-L. Lions' lectures at Collège de France), Technical Report.

[5] CARMona,R. and DelarUe, F. (2008)Probabilistic Analysis of Mean-Field Games, Technical Report; Hal-00714589.

[6] CARmona, R., Delarue, F. and LACHAPELle, A. (2012) Control of McKean-Vlasov Dynamics versus Mean-Field Games, arXiv:1210.5771v1.

[7] HAMADÈNE, S. (1998) Backward-forward SDEs and stochastic differential games, Stochastic Processes and their Applications 77, pp.1-15.

[8] HAMADÈNE, S. (1999) Nonzero-sum linear quadratic stochastic differential games and backward forward equations, Stochastic Analysis and Appl., 17, 117-130.

[9] HAMADÈNE, S., LEPELTIER, J.-P. (1995) Backward equations, stochastic control and zero-sum stochastic differential games, Stochastics and stochastic Reports, vol.54, pp.221-231.

[10] HUANG, M. (2010) Large-population LQG games involving a major player: the Nash certainty equivalence principle, SIAM J.Control Optim., 48(5), 3318-3353.

[11] HUANG, M., MALHAME, R.P. and CAINES, P.E. (2006) Large population stochastic games: closedloop McKean-Vlasov Systems and the Nash Certainty Equivalence Principle, Comm. in Information and Systems, 6(3), 221-252.

[12] LASRY, J.M. and LIONS, P.L. (2006) Jeux à champs moyen I. Le cas stationnaire, Comptes Rendu de l'Académie des Sciences de Paris, série A, 343(9).

[13] LASRY, J.M. and LIONS, P.L. (2006) Jeux à champs moyen II. Horizon fini et contrôle optimal, Comptes Rendu de l'Académie des Sciences de Paris, série A, 343(10).

[14] LASRY, J.M. and LIONS, P.L. (2007) Mean-field games, Japanese Journal of Mathematics, 2(1).

[15] LASRY, J.M. and LIONS, P.L. (2007) Mean-field games, Cahiers de la Chaire Finance et Développement Durable (2).

[16] NOURIAN M. and CAINES,P.E. (2012) E-Nash Mean Field Game Theory for Nonlinear Stochastic Dynamical Systems with Major and Minor Agents, arXiv:1209.5684.

[17] NOURIAN M., CAINES, P.E., MALHAMÉ, R.P. and HUANG, M. (2013) Nash, Social and Centralized Solutions to Consensus Problems via Mean Field Control Theory, IEEE Transactions on Autom. Contr. $58(3), 639-653$.

[18] SZNITMAN, A.S. (1991) Topics in propagation of chaos. Lect. Notes in Math, 1464, 165-252, SpringerVerlag, Berlin. 